*For correspondence:

jefferis@mrc-Imb.cam.ac.uk (GJ)

†These authors contributed equally to this work

${ }^{\ddagger}$ These authors also contributed equally to this work

\section{Information flow, cell types and stereotypy in a full olfactory connectome}

\author{
Philipp Schlegel ${ }^{1,2 \dagger}$, Alexander S. Bates ${ }^{1 \dagger}$, Tomke Stürner ${ }^{2 \ddagger}$, Sridhar R. \\ Jagannathan $^{2 \ddagger}$, Nikolas Drummond ${ }^{2}$, Joseph $\mathrm{Hsu}^{2,3}$, Laia Serratosa Capdevila², \\ Alexandre Javier ${ }^{2}$, Elizabeth C. Marin ${ }^{2}$, Asa Barth-Maron ${ }^{4}$, Imaan F. M. Tamimi ${ }^{2}$, \\ Feng $\mathrm{Li}^{3}$, Gerald M. Rubin ${ }^{3}$, Stephen M. Plaza ${ }^{3}$, Marta Costa ${ }^{2}$, Gregory S.X.E. \\ Jefferis $^{1,2}$
}
${ }^{1}$ Neurobiology Division, MRC Laboratory of Molecular Biology, Cambridge CB2 0QH, UK; ${ }^{2}$ Department of Zoology, University of Cambridge, Cambridge CB2 3EJ, UK; ${ }^{3}$ Janelia Research Campus, Howard Hughes Medical Institute, Ashburn, United States; ${ }^{4}$ Department of Neurobiology, Harvard Medical School, Boston, United States

\section{Introduction}

By providing a full account of neurons and networks at synaptic resolution, connectomics can form and inform testable hypotheses for nervous system function. This approach is most powerful when applied at a whole-brain scale. However, until very recently, the handful of whole-brain connectomics data sets have either been restricted to complete nervous systems of a few hundred neurons (i.e. nematode worm [White et al. (1986)] and Ciona tadpole [Ryan et al. (2016)]) or to the sparse tracing of specific circuits, as in larval and adult Drosophila (Zheng et al., 2018; Ohyama et al., 2015).

Now, for the first time, it has become possible to analyse complete connectomes at the scale of the adult vinegar fly, Drosophila melanogaster. The 'hemibrain' EM data set (Scheffer et al., 2020) provides a step-change in both scale and accessibility: dense reconstruction of roughly 25,000 neurons and 20M synapses comprising approximately half of the central brain of the adult fly. The challenge now lies in extracting meaning from this vast amount of data. In this work, we develop new software, analytical tools and integration strategies, and apply them to annotate and analyse 


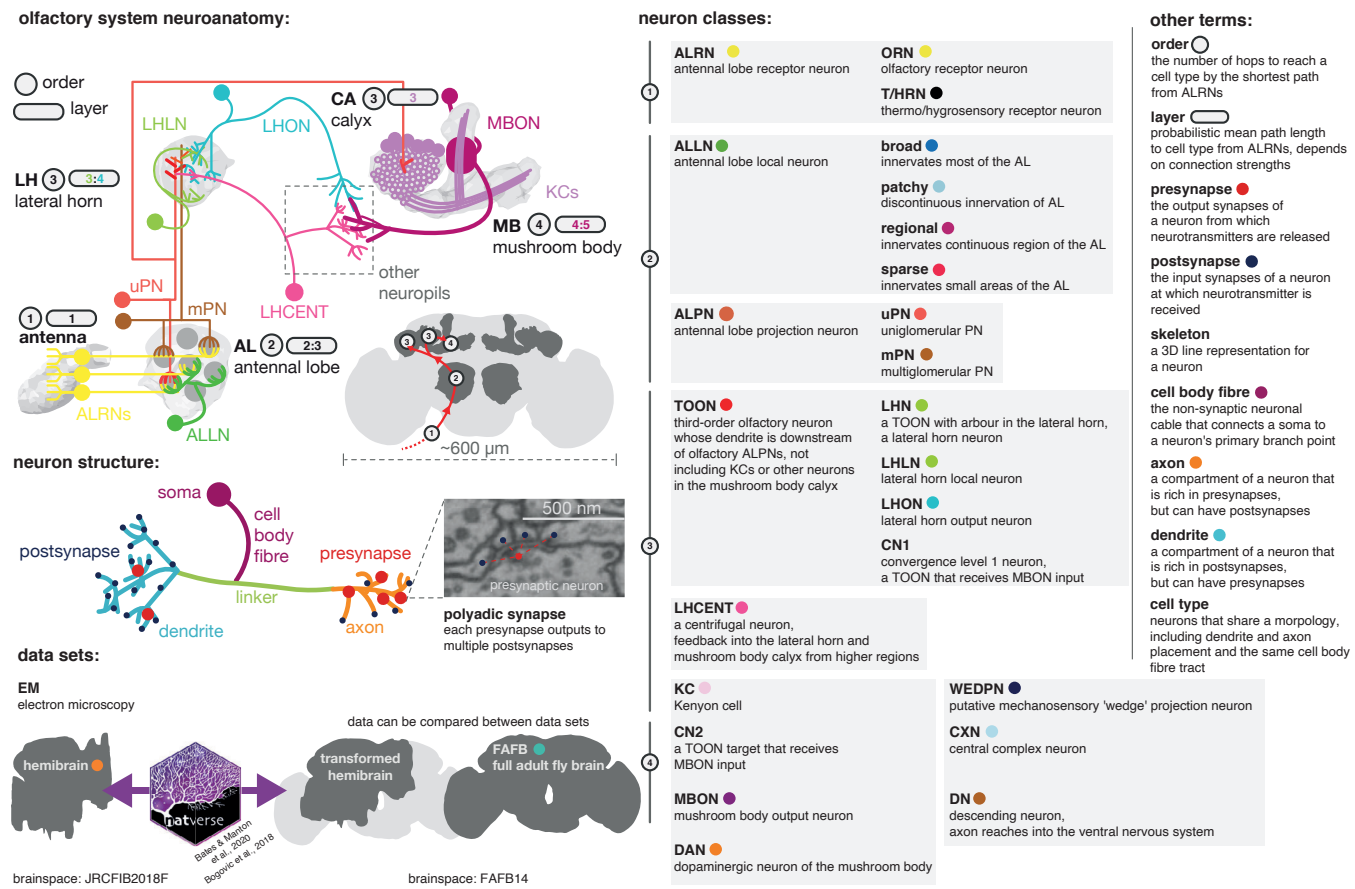

Figure 1. Graphical olfactory neuroanatomy glossary. Top left, schematic of the $D$. melanogaster olfactory system showing all its major neuron classes. The 'order' of each neuropil is given in a grey circle, its average layers in a grey lozenge. Inset, the fly brain with a scale bar and early olfactory neuropils shown. Red path is the major feedforward course of olfactory information through the brain. Middle left, a neuron with its compartments is shown. Bottom left, the two EM data sets that feature in this work, the partial dense connectome, the hemibrain, and a sparsely reconstructed data set, FAFB. Neuroanatomical data can be moved between the two spaces using a bridging registration (Bogovic et al., 2020; Bates et al., 2020a). Right, major neuron class acronyms are defined. Other neuroanatomical terms are also defined. Coloured dots indicate the colour used to signal these terms in the following figures.

Figure 1-video 1. Video of neurons typed in this study grouped by broad class. Colours correspond to cell type (ALRNs), lineage (ALRNs) or are random (ALPNs, TOONs).

The fly olfactory system is the largest central brain system that spans first-order sensory neurons to descending premotor neurons; it is a powerful model for the study of sensory processing, learning and memory, and circuit development (Amin and Lin, 2019; Groschner and Miesenböck, 2019). In this study we take a principled approach to identify both large scale information flow and discrete connectivity motifs using the densely reconstructed hemibrain data set. In addition, we compare and validate results using a second EM data set, the full adult fly brain (FAFB, Zheng et al. (2018)), which has been used until now for sparse manual circuit tracing (e.g. Dolan et al. (2018, 2019); Sayin et al. (2019); Felsenberg et al. (2018); Huoviala et al. (2018); Zheng et al. (2020); Marin et al. (2020); Bates et al. (2020b); Otto et al. (2020); Coates et al. (2020)).

We catalogue first-order receptor neurons innervating the antennal lobe, second-order neurons including all local interneurons, and a full survey of third-order olfactory neurons (excepting the mushroom body, MB, see Li et al. (2020)). This classification defines cell types and associates all olfactory neurons with extant functional knowledge in the literature, including the molecular identity of the olfactory information they receive. To further aid human investigation and reasoning in the data set, we develop a computational strategy to classify all olfactory neurons into layers based on their distance from the sensory periphery. We apply this across the full data set, for example identifying those descending neurons (connecting the brain to the ventral nerve cord) that are particularly early targets of the olfactory system. 
We also carry out focused analysis at different levels, including the antennal lobe, crucial for initial sensory processing (Wilson, 2013), where we reveal highly lateralised microcircuits. After the antennal lobe, information diverges onto two higher olfactory centres, the MB (required for learning) and the lateral horn (LH) (Vosshall and Stocker, 2007; Heisenberg, 2003; Grabe and Sachse, 2018). We analyse reconvergence downstream of these divergent projections as recent evidence suggests that this is crucial to the expression of learned behaviour (Dolan et al., 2018, 2019; Bates et al., 2020b; Eschbach et al., 2020; Kadow, 2019).

Finally, building on our recent analysis of second-order olfactory projection neurons in the FAFB data set (Bates et al., 2020b), we investigate the stereotypy of cell types and connectivity both within and across brains for select circuits. We show that in two separate cases, variability across different brains is similar to variability across the two hemispheres of the same brain. This has important practical implications for the interpretation of connectomics data but also represents a first quantitative effort to understand the individuality of brain connectomes at this scale.

\section{Results}

\section{Neurons of the olfactory system}

The Janelia hemibrain data set comprises most of the right hemisphere of the central brain of an adult female fly and contains $\sim 25,000$ largely complete neurons; neurons were automatically segmented and then proofread by humans recovering on average $\sim 39 \%$ of their synaptic connectivity (Scheffer et al., 2020). Here we process this data into a graph encompassing $12.6 \mathrm{M}$ chemical synapses across 1.7M edges (connections) between 24.6k neurons (see Methods). Leveraging this enormous amount of data represents a major challenge. One way to start understanding these data is to group neurons into broad classes and discrete cell types; this enables summaries of large scale connectivity patterns as well as linking neurons to extant anatomical, physiological and behavioural data.

As a first step, we carried out a comprehensive annotation of all first, second and third-order olfactory neurons as well as many higher-order neurons. In particular, we annotate antennal lobe olfactory and thermo/hygrosensory receptor neurons (ALRNs), uni - and multiglomerular projection neurons (UPNs, mPNs), antennal lobe local neurons (ALLNs), lateral horn neurons (LHNs) and lateral horn centrifugal neurons (LHCENT). Defining cell type annotations depended on a range of computational tools as well as expert review and curation. Broadly, we used NBLAST (Costa et al., 2016) to cluster neurons into morphological groups and cross-reference them with existing lightlevel data and in many cases confirmed typing by comparison with the FAFB EM data set (Zheng et al., 2018; Dorkenwald et al., 2020).

Our annotation efforts - - amounting to 4732 cells and 966 types - - were coordinated with those of Kei Ito, Masayoshi Ito and Shin-ya Takemura, who carried out cell typing across the entire hemibrain EM data set (Scheffer et al., 2020). Other typing efforts are reported in detail elsewhere (see e.g. Li et al. (2020) for Kenyon cells, KCs; mushroom body output neurons, MBONs; dopaminergic neurons, DANs; Hulse et al. (2020) for neurons of the central complex; CXN) (Figure 2A,B). All cell type annotations agreed upon by this consortium have already been made available through the hemibrain v1.1 data release at neu print.janelia.org in May 2020 (Scheffer et al., 2020; Clements et al., 2020).

Owing to the truncated nature of the hemibrain EM volume, descending neurons (DNs) are particularly hard to identify with certainty. By careful review and comparison with other data sets including the full brain FAFB data set, we identified 236 additional DNs beyond the 109 reported in the hemibrain v1.1 release (see Methods and Supplemental Data).

\section{Layers in the olfactory system}

Having defined cell types of the olfactory system, a second approach to obtain a system wide understanding of olfactory organisation is to characterise the connectome graph with respect to an 

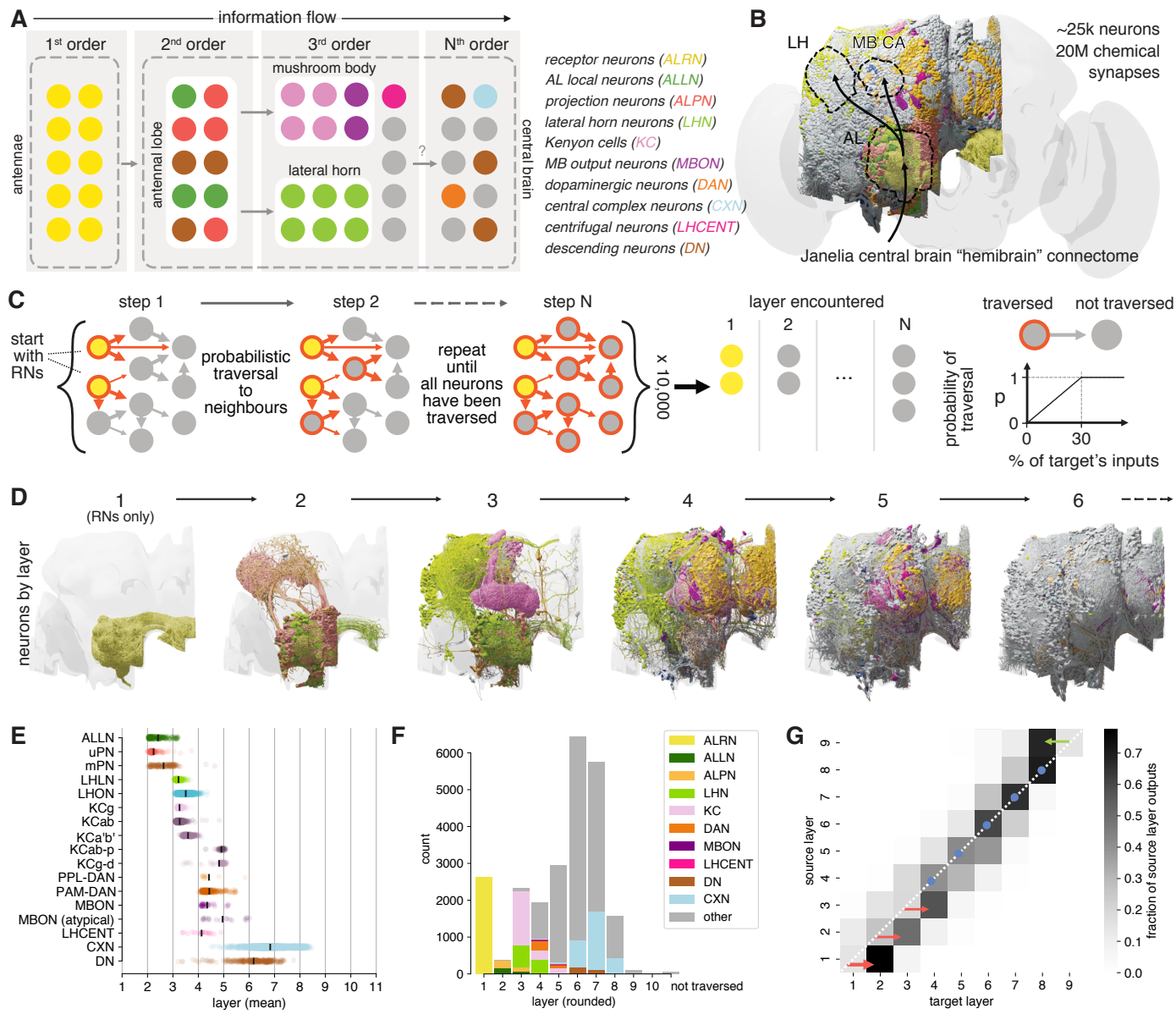

$\mathbf{F}$
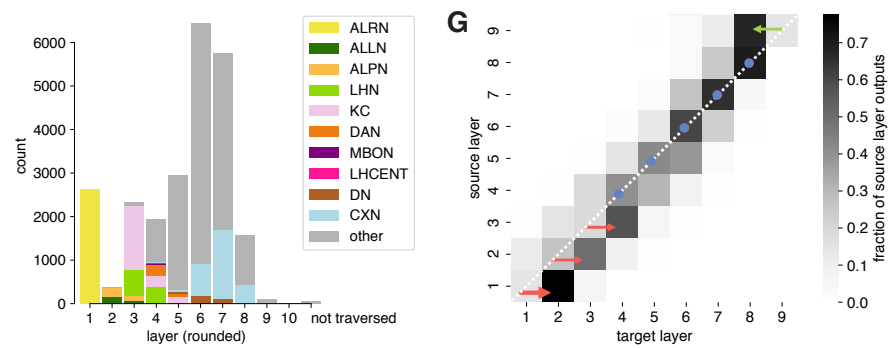

Figure 2. Identification of layers in the olfactory system. A Schematic of the fly's olfactory system. Colours reused in subsequent panels. B The Janelia Research Campus FlyEM hemibrain connectome. Principal olfactory neuropils as overlay; full brain plotted for reference. C Graph traversal model used to assign layers to individual neurons. D Neurons found in the first six layers. E Mean layer of individual neurons. Black line represents mean across a given neuron class. $\mathbf{F}$ Composition of each layer. $\mathbf{G}$ Connections between layers. Abbreviations: AL, antennal lobe; CA, calyx; LH, lateral horn; MB, mushroom body; WEDPN, wedge; ALPN, antennal lobe projection neuron; uPN/mPN, uni-/multiglomerular ALPN.

Figure 2-video 1. Video of neurons of the first 5 olfactory layers. Colours correspond to neuron types (e.g. ALRNs, ALPNs, etc) also used elsewhere.

Figure 2-Figure supplement 1. Graph traversal model extended data.

Figure 2-Figure supplement 2. Olfactory vs thermo/hygrosensory layers.

inferred sensory-integrative-motor hierarchy. While this cannot model all aspects of brain function it provides a human-intelligible summary of information flow.

The basic organisation of the early fly olfactory system is well documented and can be summarised as follows: first order receptor neurons (ALRNs) in the antennae project to the brain where they terminate in the antennal lobes (AL) and connect to second-order local (ALLNs) and projection neurons (ALPNS). Information is then relayed to third-order olfactory neurons mainly in the mushroom body (MB) and the lateral horn (LH) (Figure 2A) (Wilson, 2013; Bates et al., 2020b). This coarse ordering of first, second and third-order neurons is helpful for neuroscientists, but is an oversimplification that has not yet been derived from quantitative analysis. The recent hemibrain dense connectome covers nearly all (known) olfactory neurons; we can therefore for the first time take a systematic approach to layering in this sensory system (Figure 2B) (Scheffer et al., 2020). Here, we employ a simple probabilistic graph traversal model to "step" through the olfactory system and 
record the position at which a given neuron is encountered. We call the positions established by this procedure "layers" to disambiguate them from the well-established term "orders" used above. Conceptually, layers correspond to the mean path length from the sensory periphery to any neuron in our graph while taking account of connection strengths; a corresponding quantitative definition of "orders" would be the shortest path length (which would not consider connection strengths).

In brief, we use the 2600 ALRNs whose axons terminate in the right antennal lobe as a seed pool (see next section and Methods for details of ALRN identification). The model then traverses to neurons downstream of those in the seed pool in a probabilistic manner: the likelihood of a given neuron being visited increases with the fraction of inputs it receives from neurons in the pool and caps at 30\%. For example, a neuron that receives 30\%/10\%/2\% of its synaptic inputs from an ALRN has a 100\%/33.3\%/0.06\% chance to be traversed in the first round. When a neuron is successfully traversed it is added to the pool and the process is repeated until the entire graph has been traversed. For each neuron, we keep track of at which step it was traversed and use the mean over 10,000 runs to calculate its layer (Figure 2C). The probability of traversal is the only free parameter in the model and was tuned empirically using well-known cell types such as uPNs and KCs. While absolute layers depended strongly on this parameterisation, relative layers (e.g. layers of uPNs vs mPNs) were stable (see Methods and Figure Supplement 1A,B for details).

Running this model on the hemibrain graph set enabled us to assign a layer to $\sim 25,000$ neurons (Figure 2D). While forgoing many of the complexities of real neural networks such as the sign (i.e. excitation vs inhibition) or types (e.g. axo-dendritic vs axo-axonic) of connections, it represents a useful simplification to quantitatively define olfactory information flow across the brain, even in deep layers far from the sensory periphery. Practically, these layers also provided a means to validate and refine the naturally iterative process of neuron classification. Early neuron classes are assigned to layers that are intuitively 'correct': for example, most ALPNs and ALLNs appear as expected in the second layer. However close inspection revealed marked differences, some of which we analyse in-depth in subsequent sections. Initial observations include the fact that mPNs appear, on average, slightly later than their uniglomerular counterparts (Figure 2E, F). This is likely due to $\mathrm{mPNs}$ receiving significant input from other second-order neurons (i.e. uPNs and ALLNs) in addition to their direct input from receptor neurons.

Neurons traditionally seen as third-order components of the two arms of the olfactory system (Kenyon cells, KCs, in the MB calyx and lateral horn neurons, LHNs) actually span two layers (3 and 4 ) due to lateral connections. Among the $\mathrm{KCS}$, those with primarily visual inputs (KC- $\alpha \beta \mathrm{p}$ and $\mathrm{KC} \gamma$-d) appear later than those with primarily olfactory input.

Descending neurons (DNs) are few ( 350-600/hemisphere) and represent the principal connection to the motor centres in the ventral nervous system (Hsu and Bhandawat, 2016; Namiki et al., 2018). We find that the majority of DNs are distant from olfactory inputs (6th layer). However, a small subset appear as third or fourth-layer neurons. These may represent shortcuts between the olfactory and motor systems used for behaviours that are hard-wired or require fast responses.

In layers 1 through 3, neurons talk primarily to others in the next higher layer (Figure 2G). Layers 4 to 7 then show increased intra-layer connectivity. At layer 6 the directionality begins to reverse: layers start connecting more strongly to neurons in the same layer and eventually the previous one(s). This may indicate that the flow of information inverts at this point and that layers 6-7 represents the "deepest" point of the olfactory system.

The above analysis combines olfactory and thermo - and hygrosensory ALRNs (see Figure 2Figure Supplement 2 for a separate break down). We will use these layers as we proceed through the olfactory system, classifying neurons in detail and extracting connectivity motifs.

\section{Antennal lobe receptor neurons}

ALRNs that express the same receptor project to the same globular compartments, glomeruli, of the olfactory bulb in vertebrates (Su et al., 2009), or the antennal lobe in insects (Couto et al., 2005; Fishilevich and Vosshall, 2005; Vosshall et al., 2000). In Drosophila, ALRNs are either unilateral or 
(more commonly) bilateral and connect with ALLNs and ALPNs (Figure 3A). We identified $\sim 2600$ ALRNs in the hemibrain data set as projecting to one of 58 glomeruli of the right antennal lobe by manually curating a list of candidate neurons (Figure 3B, see Methods for details). Notably, we renamed 3 glomeruli to resolve conflicting information in past literature: VC5 $\rightarrow$ VM6, VC3m $\rightarrow$ VC5, VC3I $\rightarrow$ VC3 (see Methods for details). These changes will appear in version 1.3 of the hemibrain dataset and have been coordinated with other research groups working on these glomeruli (Task et al., 2020; Vulpe et al., 2021)

19 glomeruli are either medially or anteriorly truncated in the hemibrain volume, while an additional 8 glomeruli are intact but have very fragmented ALRNs. This affects our recovery and identification of ALRNs (Figure 3-Figure Supplement 1A,B) and we estimate our coverage per glomerulus to be on average around 70\% compared to previously published counts (Rybak et al., 2016; Tobin et al., 2017; Horne et al., 2018; Stocker, 2001; Grabe et al., 2016) (Figure 3-Figure Supplement 1C). In subsequent analysis, ALRNs of truncated glomeruli are not included. The 31 fully intact glomeruli include all the thermo - and hygrosensory ones ( $n=7)$ and 24 olfactory ones (Figure 3C) (Marin et al., 2020; Bates et al., 2020b). Thermo - and hygrosensory ALRNs (TRN/HRNs) are mostly unilateral (6/7) with 8 ALRNs per type on average, while olfactory ALRNs (ORNs) are predominantly bilateral (22/24) with 27 ALRNs per type (Figure 3-Figure Supplement 1A).

Building on our comprehensive analysis of ALRNs, we have now found that ALRNs of the VM6 glomerulus consist of three anatomically distinct sub-populations (VM6v, VM6m and VM6I) connecting to the same postsynaptic PNs; these populations differ in their receptor expression and their origin in peripheral sense organs (Task et al., 2020; Vulpe et al., 2021). These findings helped to explain previous uncertainties about this part of the antennal lobe, which have resulted in many nomenclature discrepancies in the prior literature. Having an almost full set of ALRNs in the hemibrain, we asked whether any other glomerulus showed a similar subdivision. Based on morphological clustering, we can confirm the VM6 subpartition but also conclude that none of the other glomeruli exhibit a similar potential for further partitioning (Figure 3-Figure Supplement 2AB) Moreover, we find that the VM6 ALRN subpopulations, while morphologically distinct, appear to converge onto the same downstream targets. None of the uniglomerular ALPNs show a clear preference towards any individual VM6 ALRN subtype. Likewise, a clustering of VM6 ALRNs based on their downstream connectivity does not align with the morphology based clustering ( Figure 3Figure Supplement 2C).

Besides providing the first large scale quantification of synaptic connectivity in the adult antennal lobe, we focused on two specific aspects: first, connection differences between the olfactory and thermo/hygrosensory ALRNs; second, wiring differences between ALRNs originating from the ipsilateral and contralateral antennae. Most of the output from ALRNs is to ALLNs (43\% and 48\% from ORNs and TRN/HRNs, respectively), followed by ALPNs (34\% and 41\% from ORNs and TRNs/HRNs, respectively). The remainder is either accounted for by ALRN-ALRN connectivity or other targets that are not ALRNs, ALPNs, or ALLNs. This connectivity profile is similar to what has been reported for the larva (Berck et al., 2016) even though the number of neurons and types has increased significantly (Scheffer et al., 2020; Bates et al., 2020b). They are also consistent with two previous studies of single glomeruli in the adult fly (Horne et al., 2018; Tobin et al., 2017).

We find that compared to ORNs, TRN/HRNs spend more of their output budget on connections to ALPNs (41\% vs 34\%) and this difference seems to be mostly accounted for by the very low level of axo-axonic TRN/HRN to ALRN connectivity (Figure 3D). Type specificity is also clearly apparent, however, with individual ALRN types showing different presynaptic densities (Figure 3Figure supplement 1D) as well as particular profiles of ALLN and ALPN output (Figure 3D-E). Two pheromone-sensitive ORN types, DA1 and VA1v, output the most to other neurons. Their main target are the AL-AST1 neurons which arborise in and receive input from a subset of antennal lobe glomeruli and output mostly in the antennal lobe and the saddle, a region that includes the antennal mechanosensory and motor centre (AMMC) (Scheffer et al., 2020; Tanaka et al., 2012a).

The majority of input onto ALRNs is from ALLNs and other ALRNs, and can vary widely - in 
particular across the different ORN types (Figure 3F). Connections between ALRNs occur almost exclusively between neurons of the same type, e.g. DA1 $\rightarrow$ DA1 but not DA1 $\rightarrow$ DA2 (data not shown). This is consistent with previous reports of connections between axon terminals of gustatory or mechanosensory neurons in larval and adult Drosophila (Hampel et al., 2020; Miroschnikow et al., 2018). The functional relevance of these connections is unclear. In contrast, ALLNs have been shown to regulate and coordinate activity across glomeruli via lateral inhibition (see for example Mohamed et al. (2019); Wilson and Laurent (2005)) and ALLN $\rightarrow$ ALRN connections likely play a role. We find that pheromone-sensitive ORNs (targeting DA1, DL3 and VA1v) are amongst those with the least ALLN input onto their terminals, suggesting that they might be less strongly modulated by other channels. As expected from analysis of output connectivity, TRNs and HRNs mostly receive input from ALLNs.

Breaking down bilateral ORN connectivity by laterality highlights a distinct behaviour of ALLNs: on average, contralateral ORNs provide more information to, and receive more information from, ALLNs than ipsilateral ORNs (Figure 3E, G and Figure 3-Figure Supplement 1E). This is in contrast to ALPNs, whose behaviour is consistent with previous reports (Gaudry et al., 2013; Agarwal and Isacoff, 2011). This bias could help the animal to respond to lateralised odour sources.

\section{Antennal lobe local neurons}

Light microscopy studies have estimated 200 antennal lobe local neurons (ALLNs) (Chou et al., 2010). ALLNs have complex inhibitory or excitatory synaptic interactions with all other neuron types in the antennal lobe, i.e. the dendrites of outgoing ALPNs, the axons of incoming ALRNs and other ALLNs. In particular, ALLN-ALLN connections are thought to facilitate communication across glomeruli, implementing gain control for fine-tuning of olfactory behaviour (Root et al., 2008; Olsen and Wilson, 2008). ALLNs are diverse in morphology, connectivity, firing patterns and neurotransmitter profiles and critically, in the adult fly brain, they do not appear to be completely stereotyped between individuals (Seki et al., 2010; Okada et al., 2009; Chou et al., 2010; Berck et al., 2016). Previously, six types of ALLNs (LN1-LN6) had been defined mainly based on the expression of specific GAL4 lines (Tanaka et al., 2012a). The hemibrain data set now provides us with the first opportunity to identify and analyse a complete set of ALLNs at single-cell resolution.

We find 196 ALLNs in the right hemisphere which we assign to 5 lineages, 4 morphological classes, 25 anatomical groups and 74 cell types (Figure 4A-D and Figure 5-Figure Supplement 1). ALLNs derive from three main neuroblast clones: the lateral neuroblast lineage ("I" and "I2" from $A L I 1)$, the ventral neuroblast lineage (" $v$ " from ALV1) and the ventral ALLN specific lineage ("V2" from ALV2) (Sen et al., 2014). Their cell bodies cluster dorsolateral, ventromedial or ventrolateral to the antennal lobe or in the gnathal ganglion (referred to as il3 (Bates et al., 2020b; Shang et al., 2007; Tanaka et al., 2012a). Around 40\% (78) of the ALLNs are bilateral and also project to the left antennal lobe; most of these (49) originate from the v2 lineage. Correspondingly, we identified fragments of 88 ALLNs that originate in the left and project to the right antennal lobe (Figure 4A).

The morphological classification of ALLNs is based on their glomerular innervation patterns reported by Chou et al. 2010: "broad" ALLNs innervate all or most of the AL; "patchy" ALLNs exhibit characteristic discontinuous innervation; "regional" ALLNs innervate large continuous regions of the $A L$, and "sparse" ALLNs innervate only a small area of the antennal lobe (Figure 4B). These differences in innervation patterns can be quantified: for each ALLN we ranked glomeruli by the number of synapses placed inside (descending) and further normalised them per ALLN. Finally we summed those numbers up cumulatively per ALLN. Sparse ALLNs place their synapses in a select few glomeruli (typically $<10$ ), while broad ALLNs distribute their synapses evenly across the majority of glomeruli (typically $>30$ ) (Figure 4E). Anatomical groups are then defined as sets of cell types with similar morphological features.

Previous research has shown that while most ALLNs exhibit input and outputs in all innervated glomeruli, some show signs of polarisation (Chou et al., 2010). Indeed, regional and sparse ALLNs can mostly be split into an axonic and a dendritic compartment, while broad and patchy ALLNs tend 
bioRxiv preprint doi: https://doi.org/10.1101/2020.12.15.401257; this version posted May 14, 2021. The copyright holder for this preprint (which was not certified by peer review) is the author/funder, who has granted bioRxiv a license to display the preprint in perpetuity. It is made available under aCC-BY 4.0 International license.
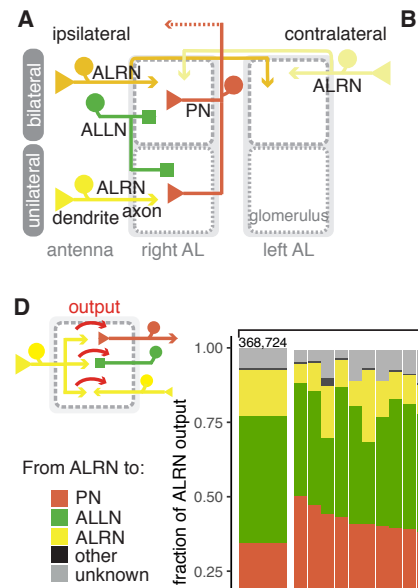

ALRN
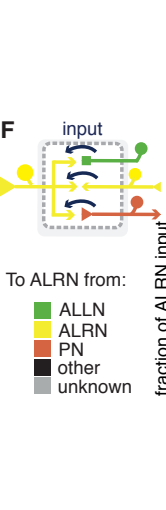
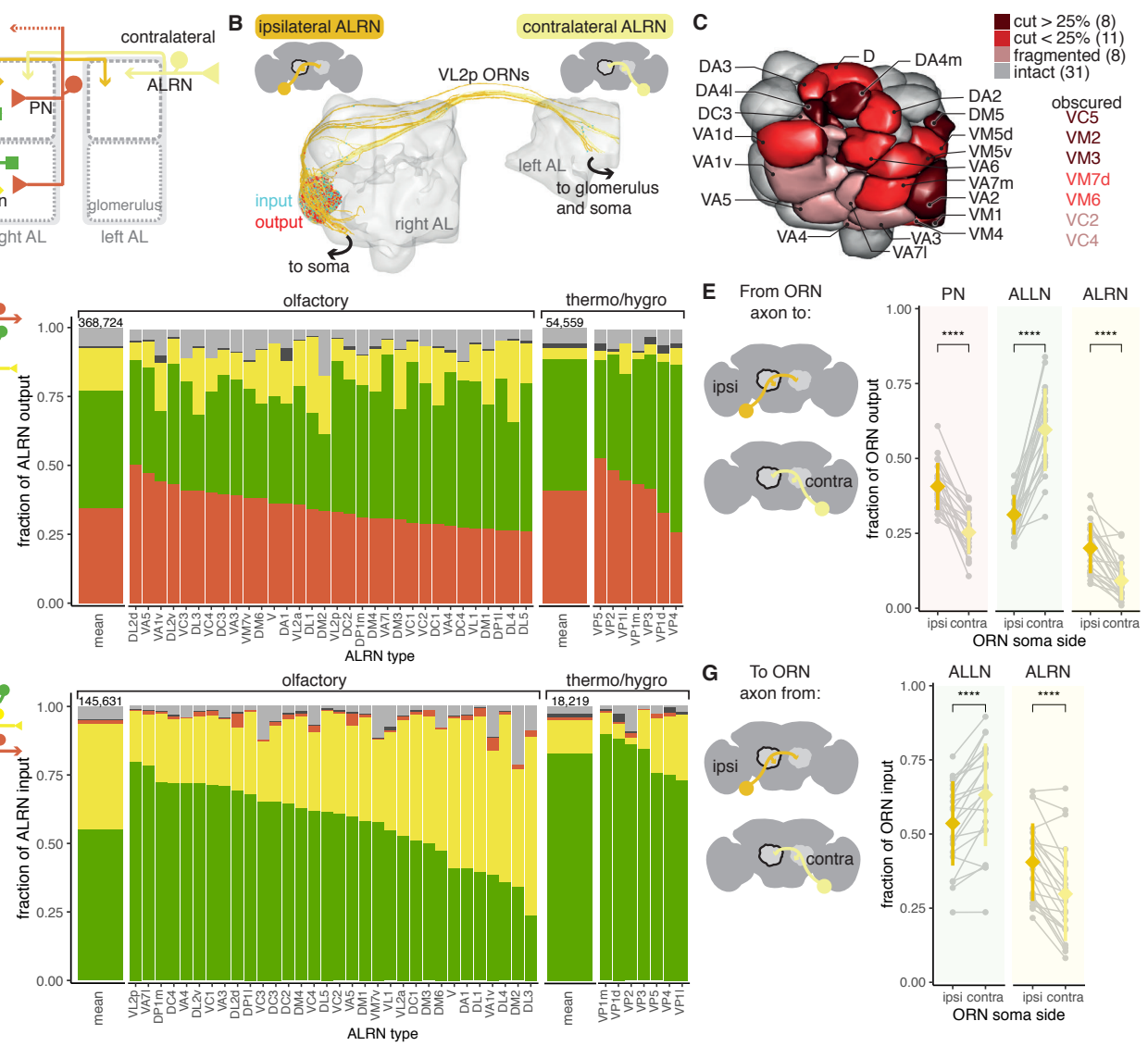

Figure 3. Antennal lobe receptor neurons mostly target projection and local neurons. A Summary schematic of antennal lobe ALRN classification and the major cell types present in the antennal lobe that interact with them. ALLN: antennal lobe local neuron; ALPN: projection neuron. B Ipsilateral and contralateral VL2p olfactory ALRNs (ORNs) in the right antennal lobe. The somas are not visible as they are cut off from the volume. Output synapses in red, input ones in blue. C Antennal lobe glomerular meshes (generated from ALRNs) showing which glomeruli are truncated and by how much (qualitative assessment). ALRN types in whole glomeruli but with fragmented ALRNs, which prevents assignment of soma side, are also shown. D Fraction of ALRN output per type. The left-most bar is the mean for olfactory or thermo/hygrosensory ALRNs, with number of synapses on top. E Fraction of ipsilateral (ipsi) or contralateral (contra) ORN output to ALLNs, ALPNs and ALRNs. Means were compared using Wilcoxon two-sample tests. F Fraction of ALRN input per type. The left-most bar is the mean for ORNs and TRNs/HRNs, with number of synapses on top. G Fraction of ipsilateral (ipsi) or contralateral (contra) ORN input from ALLNs and ALRNs. Means were compared using Wilcoxon two-sample tests. Significance values: ns: $\mathrm{p}>0.05 ; *: \mathrm{p}<=0.05 ; * *: \mathrm{p}<=0.01 ; * * *: \mathrm{p}<=0.001 ; * * * *$ : $p<=0.0001$.

Figure 3-Figure supplement 1. Annotation of ALRN bodies and connectivity features.

Figure 3-Figure supplement 2. ALRN clustering and subdivision of the VM6 glomerulus.

to be less polarised (Figure 4F). Axon-dendrite segregation may facilitate specific inter-glomerular interactions. In particular, looking at the most polarised ALLNs (score $>0.1$ ), differential dendritic input and axonic output are apparent with respect to pairs of thermo/hygrosensory glomeruli of opposing valences (Figure 4-Figure Supplement 1G). Significantly, v2LN49 neurons receive dendritic input in the 'heating' glomerulus VP2 (Ni et al., 2013), and have axonic outputs in the 'cooling' glomerulus VP3 (Gallio et al., 2011; Budelli et al., 2019), while I2LN20 and I2LN21 perform the opposite operation. An interesting odour example is ILN17 which receives dendritic inputs from pheromone glomerulus DA1 and has axonic output to another pheromone glomerulus, VA1v (Kurtovic et al., 2007; Dweck et al., 2015). Such interactions might help regulate female receptivity.

ALLNs principally connect to ALRNs, ALPNs and other ALLNs. Connectivity differs greatly be- 
bioRxiv preprint doi: https://doi.org/10.1101/2020.12.15.401257; this version posted May 14, 2021. The copyright holder for this preprint (which was not certified by peer review) is the author/funder, who has granted bioRxiv a license to display the preprint in perpetuity. It is made available under aCC-BY 4.0 International license.
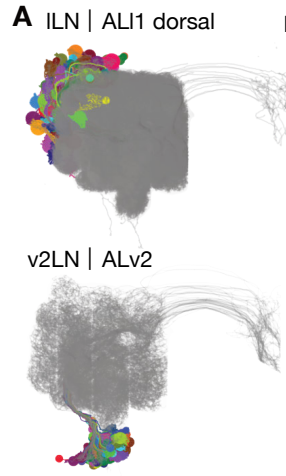

il3LN | -
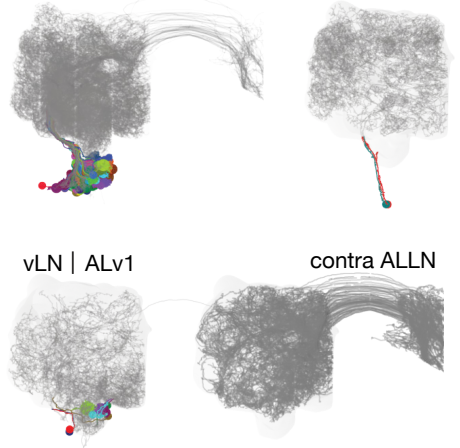

B

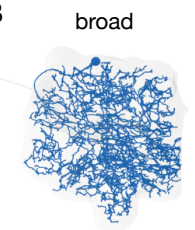

regional

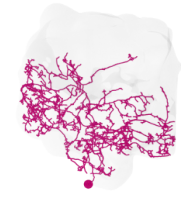

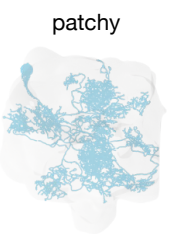

sparse

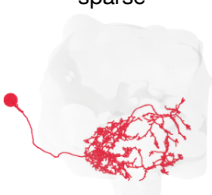

D dense vs weak

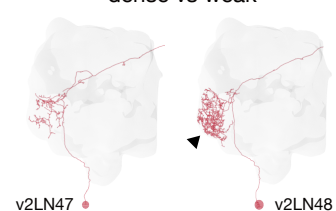

unilateral vs bilateral
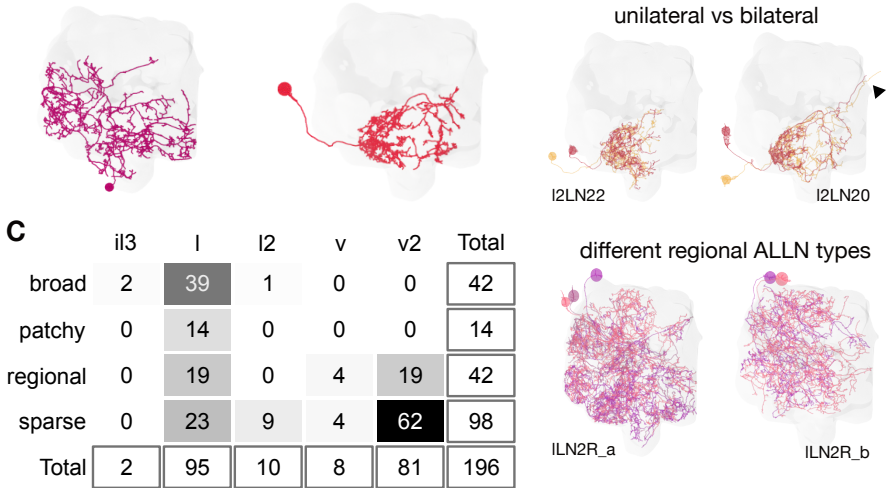

different regional ALLN types

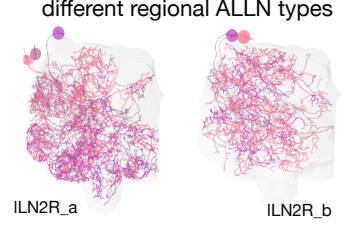

E

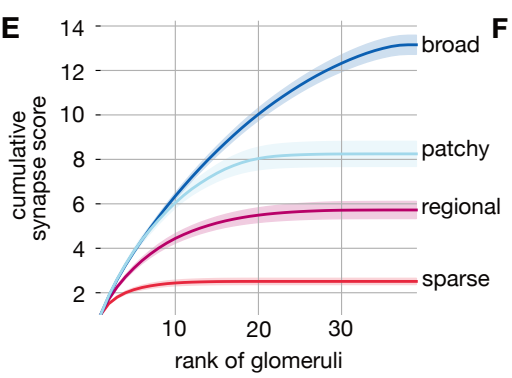

$\mathbf{F}$

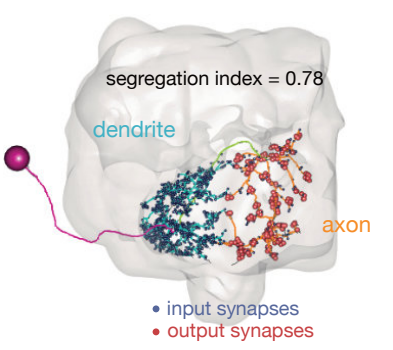

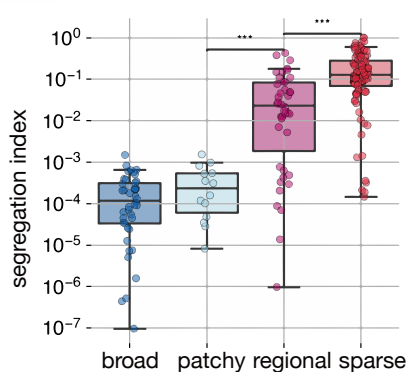

Figure 4. Cell typing, morphological classification and polarity of antennal lobe local neurons. A ALLNS classified by hemilineage and contralateral ALLNs (contra ALLN), along with the antennal lobe mesh in the background. Soma locations (circles) and primary neurite tracts are illustrated in multicolours. B Morphological classes of ALLNs. A representative example of each category is shown. C Number of ALLNs per hemilineage and morphological class. D Representative examples illustrating criteria used for typing: unilateral and bilateral neurites, lineage identity, area innervated by ALLN neurites and their density. Arrow heads point towards dense innervation and bilateral projection. E Synapse score per morphological class. Cumulative number of synapses is computed per ranked glomerulus (by number of synapses) and plotted against its rank. Envelopes represent standard error of the mean. $\mathbf{F}$ Polarisation of neurites per morphological class. Segregation index is a metric for how polarised a neuron is; the higher the score the more polarised the neuron (Schneider-Mizell et al., 2016). Left inset shows a sparse ALLN, I2LN21, as an example of a highly polarised ALLN. Significance values: $*$ : $p<=0.05 ; * *$ : $p<=0.01 ; * * *$ : $p<=0.001 ; * * * *$ : $p<=$ 0.0001; pairwise Tukey-HSD post-hoc test.

Figure 4-Figure supplement 1. ALLN glomerular innervation patterns.

tween ALLN cell types, even within groups (Figure 5A,B). Smaller ALLNs (sparse, regional) tend to receive a greater fraction of direct ALRN input than larger ALLNs (broad, patchy) and are therefore assigned to earlier layers (Figure 5A). Strong ALLN-ALLN connectivity arises mostly from the broad ALLNs of the lateral lineage (Figure $5 \mathrm{C}$ ). They may act as master regulators of the ALLN network.

Breaking down the input onto ALLNs, we see that some have very high specificity for specific glomeruli: for example, vLN24 receives $67 \%$ of its ALRN input from the $\mathrm{CO}_{2}$ responsive $V$ glomerulus ORNs (Figure 5B,F). Importantly, we also observe substantial differences in the degree of ipsi - versus contralateral ALRN input across the ALLN population (Figure 5B). At one end of the spectrum, regional vLNs receive more than 10 times as much input from ipsilateral versus contralateral ORNs; in contrast broad il3LNs receive fivefold more contralateral ORN input. These broad il3LNs, a single pair of bilateral neurons likely analogous to the larval Keystone ALLNs (Coates et al., 2020; Berck et al., 2016), interact strongly with broad ILNs while also providing strong presynaptic inhi- 

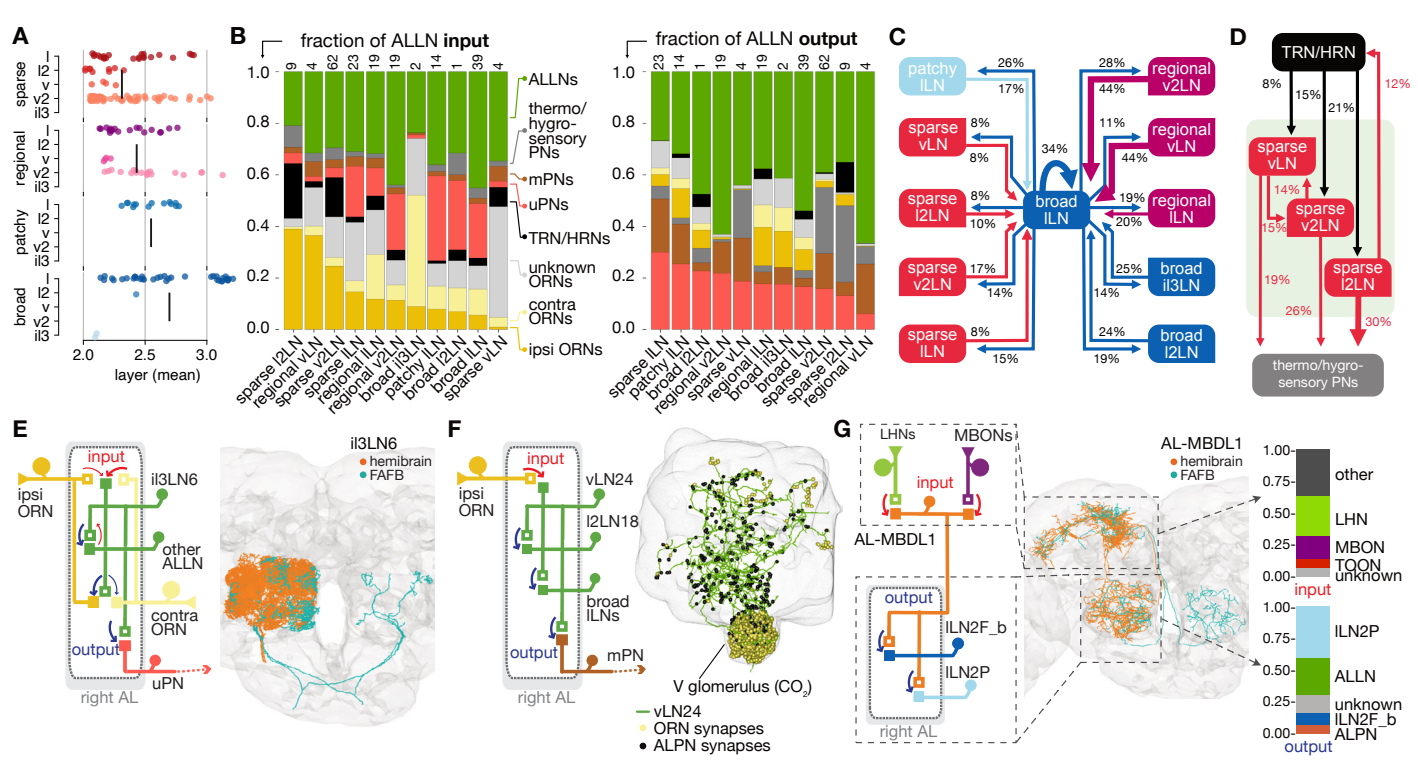

Figure 5. Antennal lobe local neuron connectivity and example circuit motifs. A Layers of ALLNs. Vertical lines indicate group mean.B Fraction of ALLN input (left) and output (right) for different ALLN groups. Number of neurons per category is shown at the top of each bar. Where possible ORNs are split into 'ipsi-' and 'contra'-lateral ('unknown' ORNs mostly correspond to those that are fragmented or belonging to truncated glomeruli). C Diagram illustrating ALLN-ALLN connectivity. ALLN groups are coloured by morphological class. D Diagram illustrating the most prominent ALLN connections to thermo/hygrosensory ALRNs and ALPNs. E-G Examples of ALLN connectivity. E A pair of broad ALLNs, il3LN6, cross-matched to the FAFB Keystone ALLNs. F An example of type regional ALLNs, vLN24, that receives specialised input in the $V$ glomerulus. G A bilateral medial bundle neuron, AL-MBDL1, that integrates LHN and MBON input and outputs to two specific types of ALLNs, broad ILN2F_b and patchy ILN2P.

Figure 5-Figure supplement 1 . Antennal lobe local neuron groups.

bition onto ORN terminals (Figure 5B,C,E). This may represent a major mechanism by which contralateral odour information influences the ipsilateral ALLN-ALLN network.

Curiously, sparse ALLN cell types receive a large proportion of their input from TRNs/HRNs (sparse I2LNs: $21 \%$, sparse V2LN: $15 \%$, sparse vLNs: $8 \%$ ). Other cell types receive at most $5 \%$. Indeed, comparing antennal lobe innervation patterns against a random null model suggests that sparse ALLNs are more likely to co-innervate thermo/hygrosensory glomeruli (Figure 4-Figure Supplement $1 \mathrm{H}$ ). Similarly, when we examine ALPN connectivity, we see that sparse ALLN cell types send a large proportion of their output to THPNs (sparse I2LNs: 30\%, sparse v2LNs: 26\%, sparse vLNs: $19 \%$ ). Other cell types receive at most $5 \%$. This indicates that sparse ALLNs may be modulating very specific thermo/hygrosensory information among the circuitry within the AL. In combination, this suggests the existence of a local network made of sparse ALLNs that encompasses only the non-olfactory, thermo/hygrosensory glomeruli.

Regional ALLNs, on the other hand, co-innervate combinations with the DP1m (responds to e.g. 3-hexanone, apple cider vinegar) or DP1l (acetic acid) glomeruli, which may be key foododours detecting glomeruli and are some of the largest in the antennal lobe. The patchy ALLNs' co-innervation does not differ from the null model, which agrees with observations from light level data (Chou et al., 2010).

About half of the ALLNs also feedback strongly onto ALRN axons. Interestingly, ALLNs of lineages $v 2$ and $v$ send very little output to the ALRNs (regional v2LNs: $1.8 \%$, sparse vLNs: $1.7 \%$, sparse v2LNs: $5.2 \%$, sparse I2LNs: $5 \%$, regional vLNs: $1.2 \%$ ) compared with other ALLNs, which spend $>16 \%$ of their outputs on ALRN axons. The ALLNs that modulate ALRN axons likely execute circuit functions distinct from those that do not, perhaps operating to quickly adapt and stabilise ALRN responses. 
bioRxiv preprint doi: https://doi.org/10.1101/2020.12.15.401257; this version posted May 14, 2021. The copyright holder for this preprint (which was not certified by peer review) is the author/funder, who has granted bioRxiv a license to display the preprint in perpetuity. It is made available under aCC-BY 4.0 International license.

A
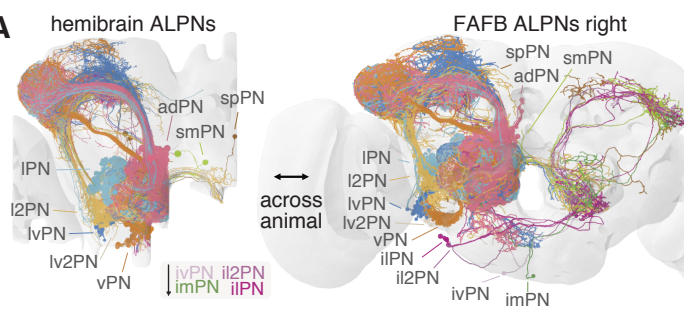

B
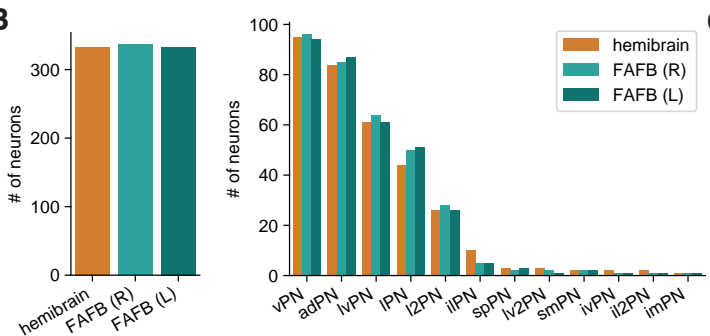

D

G

\section{Stereotypy in olfactory projection neurons}
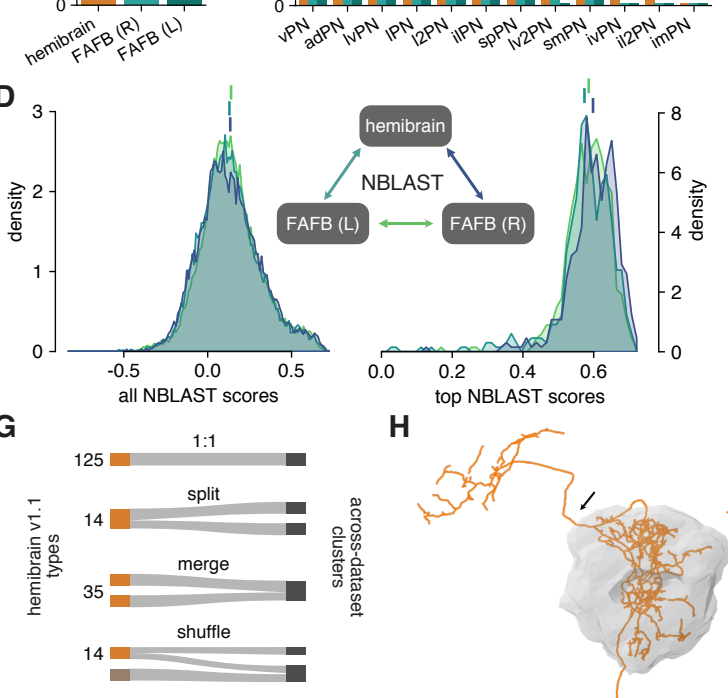

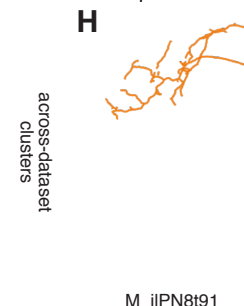

M_ilPN8t91
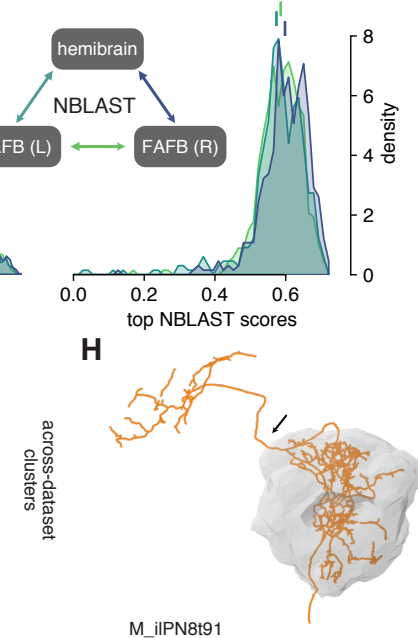

We also observe centrifugal feedback from higher olfactory areas, into the antennal lobe. The antennal lobe-associated median bundle neuron (AL-MBDL1) is a centrifugal modulatory neuron that integrates input from the MB and the LH (Tanaka et alo, 2012a) (Figure 5G). It arborises widely in the antennal lobe and outputs onto two specific sets of ALLNs: the 14 patchy ILN2P and a pair of broad ILN2F_b neurons (Figure 5F). This means that the superior brain regions may be able to exercise control over the ALLN-ALLN network through AL-MBDL1 activity.

C

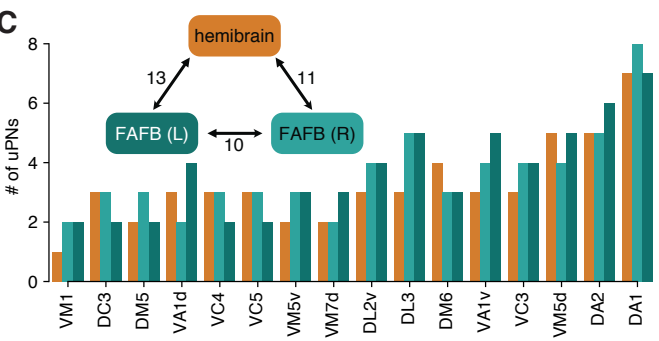

E
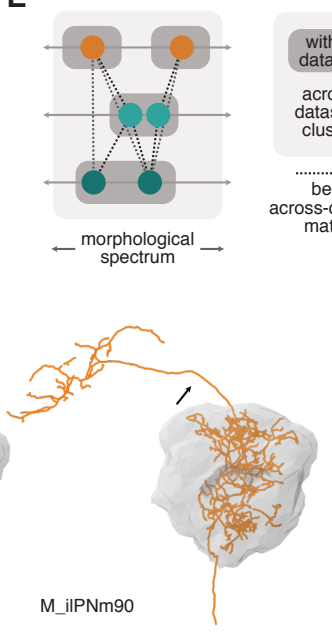

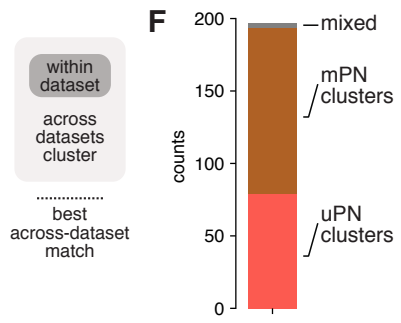

FAFB ALPNs left
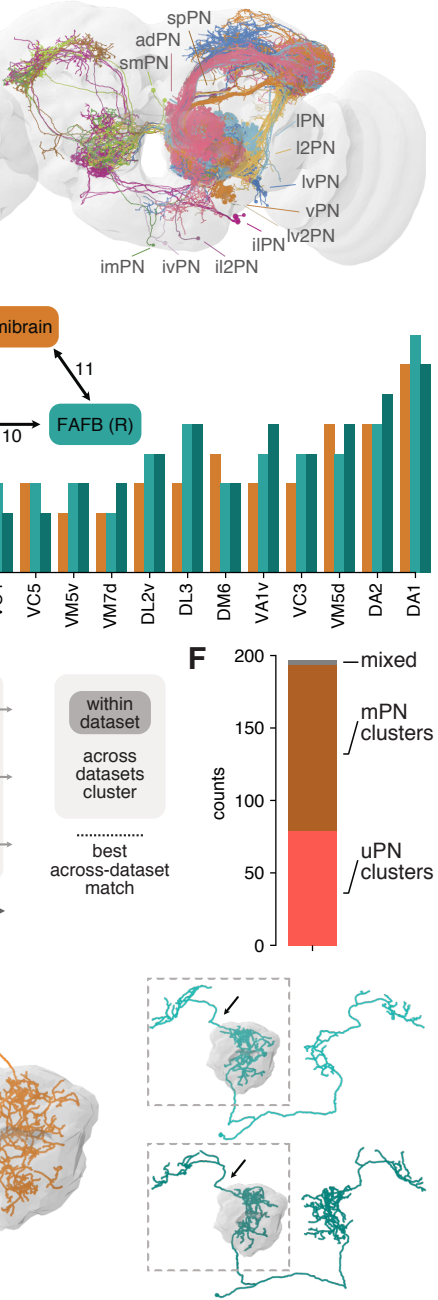

Figure 6. Numerical and morphological across - and within-animal stereotypy. A Antennal lobe projection neurons (ALPNs) reconstructed in the hemibrain and from the left and right hemispheres of the FAFB EM volume. B Overall ALPN counts are almost identical across hemispheres as well as across animals. C $17 / 56$ uPN types show variations in numbers. Numbers in triangle count instances of variation in numbers. D Across-dataset NBLAST similarity scores are much the same. All scores on the left, only pairwise top scores on the right. Top lines represent means. E Clustering approach based on best across-dataset matches. F Total number of across-dataset clusters by composition. G Quantification of discrepancies between hemibrain v1.1 types and the across-dataset clusters. See also Figure 6-Figure Supplement 1F. H Example where two hemibrain types merge into one across-dataset cluster (2). One of the hemibrain neurons takes the "wrong" antennal lobe tract (arrows) and has therefore been incorrectly given a separate type. See Figure 6-Figure Supplement 1G-J for more examples.

Figure 6-Figure supplement 1. Comparison of ALPNs across three hemispheres. 
Glomeruli are innervated by principal cells, mitral and tufted cells in vertebrates and projection neurons (ALPNs) in insects, which convey odour, temperature and humidity information to thirdorder neurons in higher brain regions (Figure 6A). These neurons may be excitatory or inhibitory, and either uniglomerular (UPNs) or multiglomerular (mPNs), i.e. sampling from a single glomerulus or multiple glomeruli, respectively (Bates et al., 2020b; Tanaka et al., 2012a).

Most uPNs are well studied and have been shown to be highly stereotyped (Jefferis et al., 2007) which makes cross-matching these cell types relatively straight-forward. In particular, the "canonical" uPN types that have been extensively studied in the past (Yu et al., 2010; Ito et al., 2013; Tanaka et al., 2012a; Grabe et al., 2016) are easily and unambiguously identifiable in the hemibrain. The situation is less clear for mPNs, for which there is as yet no conclusive cell typing. mPN types were therefore determined by the aforementioned consortium using a combination of within-dataset morphological and connectivity clustering under the assumption that these types would be further refined in future releases. In combination, hemibrain v1.1 features 188 ALPN types.

We previously described the morphology of 164 uPNs (forming 81 different types) and 181 mPNs (untyped) in the right hemisphere in the FAFB (full adult fly brain) EM volume (Bates et al., 2020b). Here, we add a third ALPN dataset from the left hemisphere of FAFB. Together, these data allow us to assess numerical and morphological stereotypy within (FAFB right vs left) and across animals (hemibrain vs FAFB left/right) (Figure 6A).

First, we find that the total number of ALPNs is largely consistent across brains as well as across hemispheres of the same brain (Figure 6B). For UPN types, we find similar variations in ALPN numbers within and across animals (Figure 6C and Figure 6-Figure Supplement 1A). Interestingly, variation only occurs in larval-born 'secondary' neurons but not with embryonic 'primary' neurons, and is more obvious for later-born neurons (Figure 6-Figure Supplement 1A).

To obtain a quantitative assessment of morphological stereotypy, we first transformed all ALPNs into the same template brain space (JRC2018F, Bogovic et al. (2020)) and mirrored the left FAFB ALPNs onto the right (see Bates et al. (2020a) and Methods for details). Next, we used NBLAST (Costa et al., 2016) to generate pairwise morphological similarity scores across the three sets of ALPNs (Figure 6D). Due to the large number of data points ( 23k per comparison), the distributions of within - and across-animal scores are statistically different $(p<0.05$, Kolmogorov-Smirnov test) however the effect size is extremely small. Importantly, the top within-animal scores are on average not higher than those from the across-animal comparisons. This suggests that neurons are as stereotyped within one brain (i.e. across left/right brain hemispheres) as they are between two brains.

An open question is whether individual cells and cell types can be recovered across animals. For neurons like the canonical uPNs this is has already been shown but it is less clear for e.g. the mPNs. First, for nearly all hemibrain ALPN we find a match in FAFB and for most neurons the top NBLAST hit is already a decent match (data not shown). The few cases without an obvious match are likely due to truncation in the hemibrain or developmental abnormalities of the neuron.

Next, we sought to reproduce hemibrain cell types across datasets. Biological variability might well produce a partition in one animal that is not present in another, and vice versa (Figure 6E). To address this, we used the top across-dataset NBLAST scores to generate 197 clusters of morphologically similar neurons across the three populations of PNs (Figure 6D-F; see Methods for details). This is slightly more than the 188 PN types listed for hemibrain v1.1 and might indicate that our approach over-segments the data. Indeed, the majority of our clusters represent 1:1:N matches (Figure 6-Figure Supplement 1B).

In general, the correspondence between hemibrain types and the across-dataset clusters is good: $74 \%$ of hemibrain types map to either one single cluster or split into separate clusters that contain only this cell type (a consequence of the over-segmentation) (Figure 6G). 35 (19\%) hemibrain types merge into larger clusters. For example, M_ilPNm90 and M_ilPN8t91 were assigned separate types because of differences in the axonal tract. In comparison with FAFB ALPNs it becomes apparent that M_ilPNm90's tract is an exception and they indeed belong to the same type 
(Figure $6 \mathrm{H})$. Only 14 ( 7\%) hemibrain types are shuffled into different clusters. We also note a few instances of discrepancies between classifications of co-clustered neurons which will be solved in future hemibrain/FAFB releases.

In summary, these results are encouraging with respect to matching neurons (types) across data sets while simultaneously illustrating potential pitfalls of cell typing based on a single dataset.

\section{Connectivity of olfactory projection neurons}

Within the antennal lobe, ALPN dendrites connect with ALRN axons and ALLNs (Figure 7A,B). As expected, olfactory mPNs and uPNs exhibit quite different connectivity profiles: mPNs receive both less overall dendritic input and also a smaller proportion of direct input from ALRNs than uPNs ( $30 \%$ vs $50 \%$ comes from ALRNs). As a consequence of these connectivity profiles, uPNs show up earlier than mPNs in the layered olfactory system (Figure 2E,F). In contrast, the connectivity profile of thermo/hygrosensory ALPNs, of which $1 / 3$ are biglomerular, is quite similar across ALPN classes, and falls in between the olfactory uPNs and mPNs (Figure 7C).

When uPNs are broken down by type, we see a range of ALRN inputs (16\% to $71 \%$ ), the majority of them from ipsilateral ALRNs (for those with bilateral ALRNs) as well as from ALLNs (15\% to $70 \%$ ) (Figure 7D). In those glomeruli with more than one uPN type, the second UPN is usually from the GABAergic vPN lineage, and receives significant input from the first, likely cholinergic uPN. vPNs (which include various multiglomerular PNs) provide feed-forward inhibition to a range of targets in the lateral horn (Bates et al., 2020b) and are thought to increase the fly's ability to discriminate (food) odors and gate between qualitatively different olfactory stimuli (Liang et al., 2013; Parnas et al., 2013). Curiously, the cholinergic V glomerulus uPN from the I2PN lineage (Bates et al., 2020b) resembles a vPN, both in terms of its output profile and total input fraction (Figure 7D,E).

Although highly polarised, olfactory uPNs have hundreds of presynapses and thousands of outgoing connections from their dendrites while mPNs make far fewer connections. Thermo/hygrosensory ALPNs have very similar output profiles to each other, although thermo/hygrosensory mPNs, as with olfactory mPNs, provide much less output in the antennal lobe. The majority of these connections are onto ALLNs (56\% to $75 \%$ ), with the remaining being onto the dendrites of other ALPNs (Figure 7F).

\section{Higher-order olfactory neurons}

The ALPN combinatorial odour code is read out by two downstream systems in very different ways. In general, the mushroom body (MB) is necessary for the formation, consolidation and retrieval of olfactory memories, while other superior neuropils support innate olfactory processing (Dubnau et al., 2001; Heimbeck et al., 2001; Krashes et al., 2007; McGuire et al., 2001; Parnas et al., 2013; Bates et al., 2020b). This dichotomy is by no means absolute (Dolan et al., 2018; Zhao et alo, 2019; Yu et al., 2004; Séjourné et alı, 2011; Sayin et al., 2019; Bräcker et al., 2013) and indeed we find numerous examples of direct interactions between these brain areas (see also Li et al. (2020)). Nevertheless, it remains a helpful simplification when investigating the logic innate vs learned pathways.

Historically third order olfactory neurons (TOONs) have often been defined by overlap with the axons of ALPNs. Using the hemibrain connectome we can now re-examine non-MB, third-order olfactory neuron morphology exhaustively. We translated this into a connectomics definition of TOONs as "neurons that receive either at least $1 \%$ (or 10 postsynapses in total) of their inputs from a single ALPN, or $10 \%$ of their inputs from any combination of ALPNs outside of the MB". This revealed a total of $\sim 2,383$ non-MB TOONs which means that both classic olfactory pathways - learned and innate - exhibit very similar convergence-divergence ratios: 2581:137:2035 ORN:PN:KC for the MB path and 2581:330:2383 ORN:PN:TOON for the non-MB path.

In the past, we focused on the lateral horn (LH) when examining TOONs in the context of innate behaviour guidance (Dolan et alo, 2019; Frechter et al., 2019), because the lateral horn is the brain neuropil most heavily innervated by ALPNs (Bates et al., 2020b). Based on light-level data, 
bioRxiv preprint doi: https://doi.org/10.1101/2020.12.15.401257; this version posted May 14, 2021. The copyright holder for this preprint (which was not certified by peer review) is the author/funder, who has granted bioRxiv a license to display the preprint in perpetuity. It is made available under aCC-BY 4.0 International license.
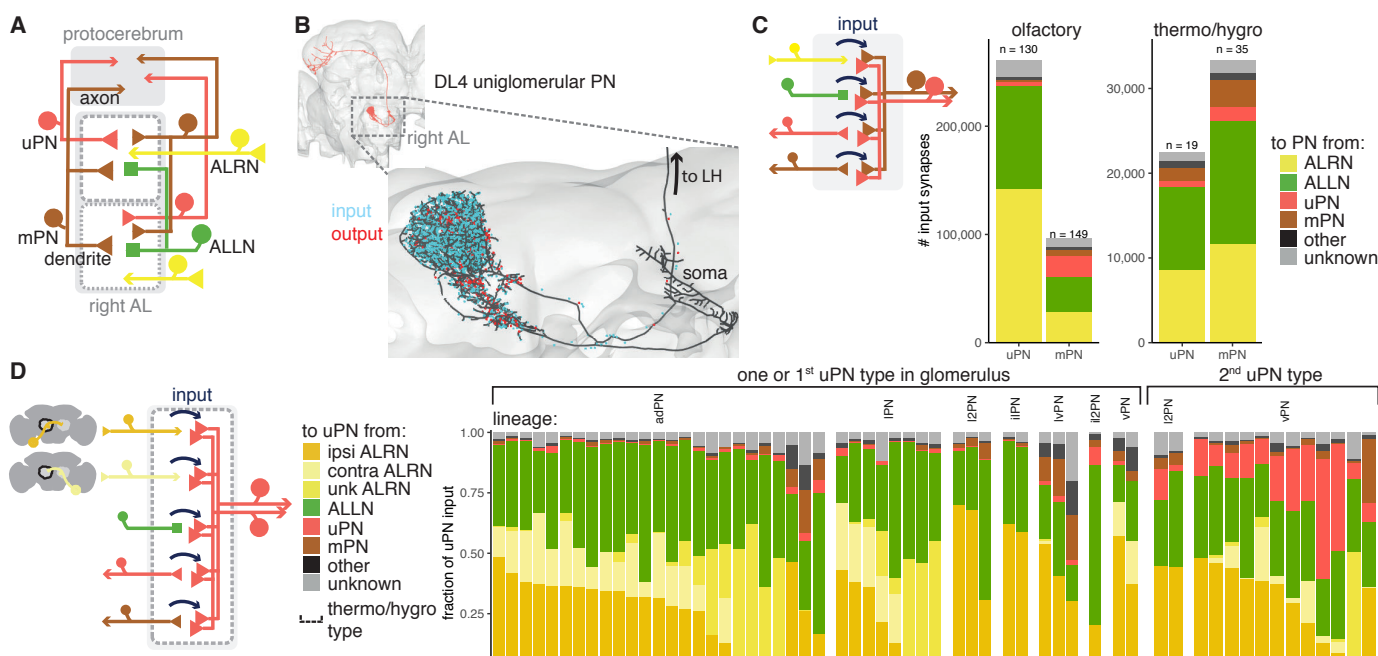

one or $1^{\text {st }} \mathrm{UPN}$ type in glomerulus
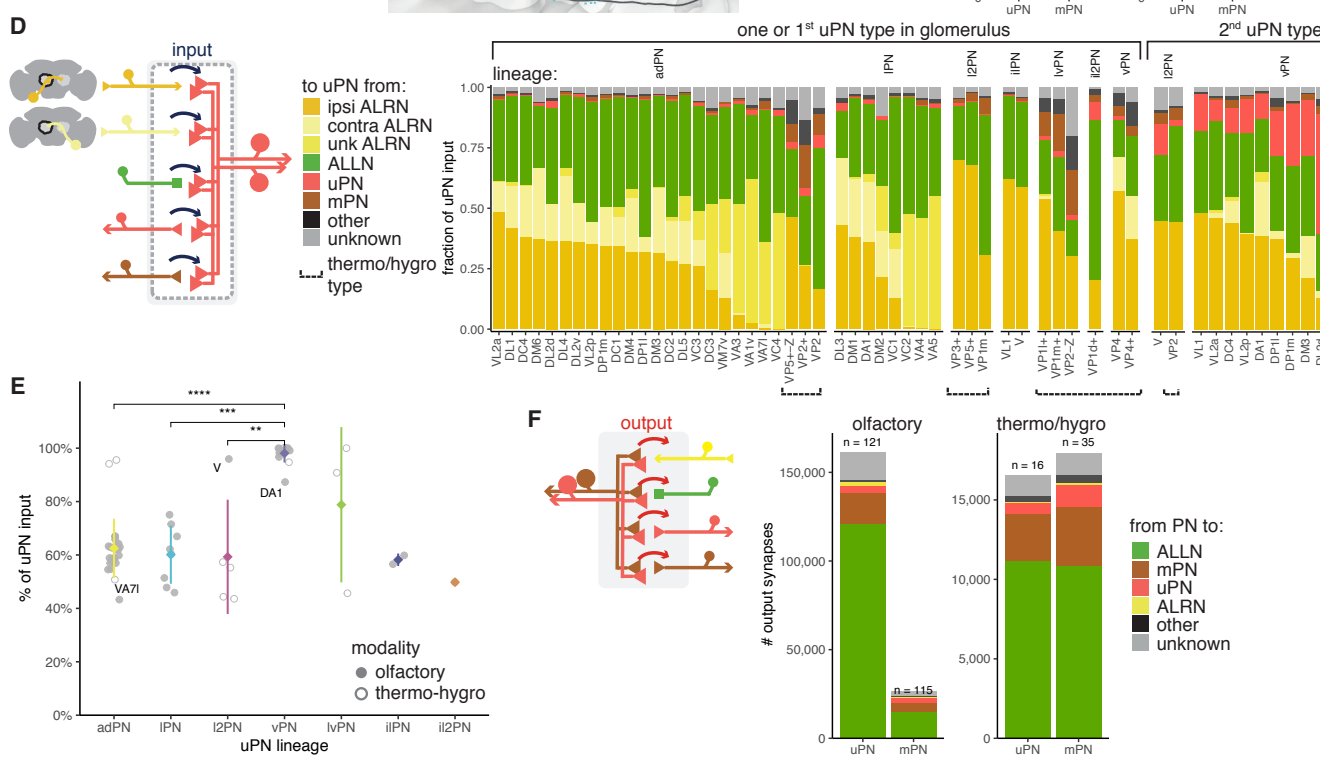

Figure 7. Antennal lobe projection neuron connectivity in the right antennal lobe. A Summary schematic of ALPN classification and the major cell types present in the antennal lobe that interact with them. UPN: uniglomerular ALPN; mPN: multiglomerular ALPN. B DL4 uniglomerular PN showing inputs (cyan) and outputs (red). C Number of input synapses onto olfactory or thermo/hygrosensory uPNs and mPNs. Number of neurons in each category shown at the top of the bar. D Fraction of UPN input, grouped by type and lineage. The left group shows glomeruli that have only one UPN type, or one of the types for those with more than one. The right group shows the second UPN type for those glomeruli with more than one. ALRN soma side indicated as 'ipsi' (ipsilateral), 'contra' (contralateral) or 'unk' (unknown, mostly corresponding to those glomeruli with fragmented ALRNs). Thermo/hygrosensory uPNs with SEZ innervation are indicated by ' $Z$ ' following the glomerulus. E Percentage of input onto uPN types relative to total connectivity (input + output), per lineage. Some of the outlier uPN types are labelled. Comparisons to categories with less than 4 data points were not done. Means per lineage were compared using Wilcoxon two sample tests. Significance values: $\mathrm{ns}: \mathrm{p}>0.05 ; *: \mathrm{p}<=0.05 ; * *: \mathrm{p}<=0.01 ; * * *: \mathrm{p}<=0.001 ; * * * *: \mathrm{p}<=0.0001$. F Number of output synapses from olfactory or thermo/hygrosensory uPNs and mPNs. Number of neurons in each category shown at the top of the bar.

we previously estimated $\sim 1,400$ third order lateral horn neuron (LHNs) forming $>264$ cell types (Frechter et al., 2019). The cell count estimate appears to have been accurate: of the hemibrain TOONs, $\sim 60 \%(1,428)$ have dendrites in the LH (Figure 8A,B) making the LH the largest target for olfactory information beyond the antennal lobe (Bates et al., 2020b). With the higher resolution of the connectome, we were able to divide these LHNs into 496 near-isomorphic cell types (Figure 8Figure Supplement $1 \mathrm{~A}$, see Methods), many of which ( 35\%) could be matched to light-level data from the literature (Frechter et al., 2019). KCs on the other hand fall into only 15 types (Li et al., 2020). Therefore, in terms of cell types, the LH path exhibits far greater expansion than the MB path (Caron et al., 2013).

The distinction between LHNs and other TOONs remains useful in that it distinguishes a subset of TOONs that are part of the densely ALPN-innervated hub that is the lateral horn. LHN cell types 
bioRxiv preprint doi: https://doi.org/10.1101/2020.12.15.401257; this version posted May 14, 2021. The copyright holder for this preprint (which was not certified by peer review) is the author/funder, who has granted bioRxiv a license to display the preprint in perpetuity. It is made available under aCC-BY 4.0 International license.

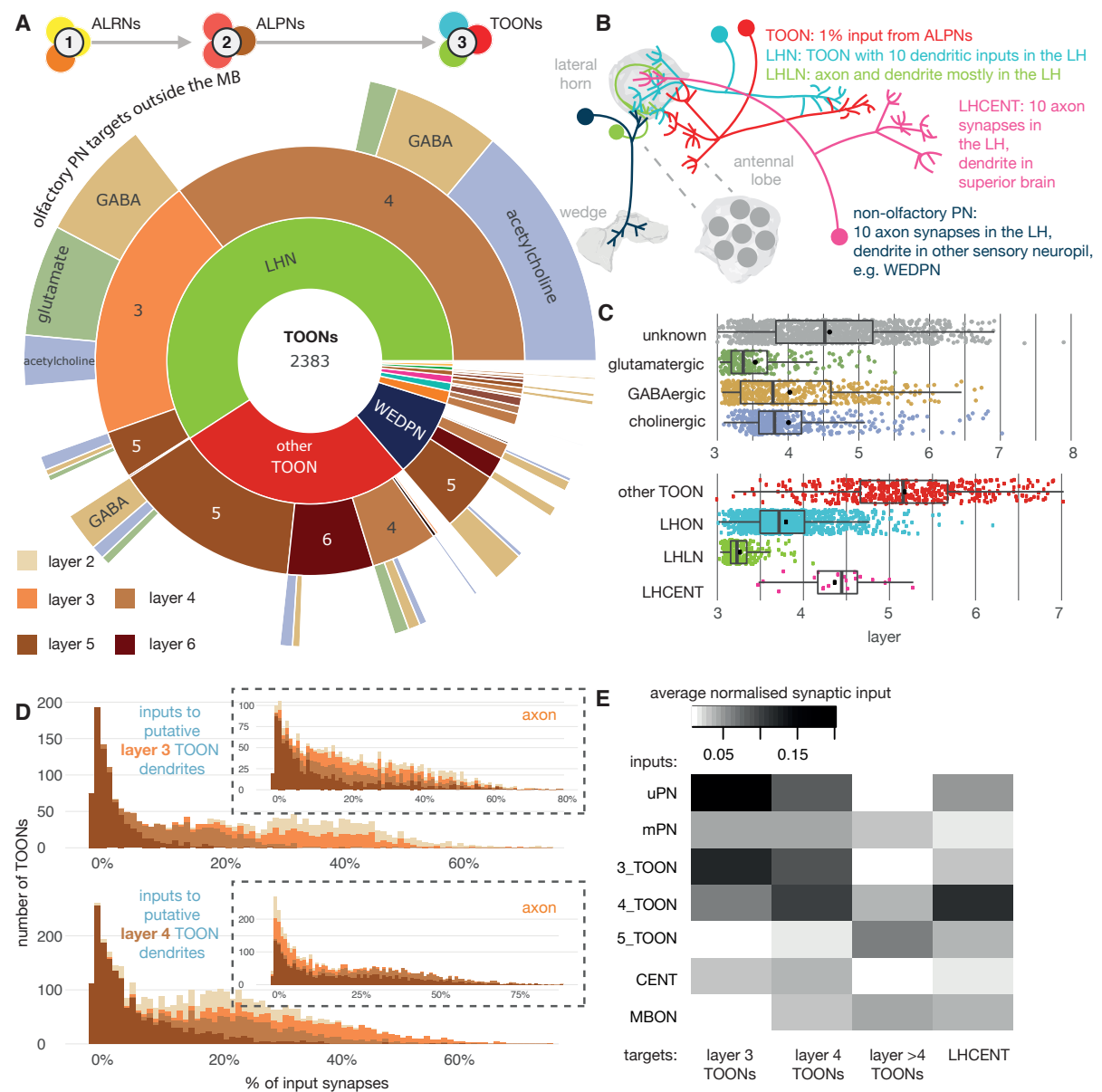

Figure 8. The targets of antennal lobe projection neuron axons. A Starburst chart breakdown of the 2,383 targets of ALPN axons, outside of the mushroom body, by various properties. We term these neurons 'third-order olfactory neurons', or 'TOONs' (see text for definition). From the inside out, neurons are grouped by: broad neuron class, layer according to the traversal model and their putative neurotransmitter. Most TOONs receive the majority of this input at their dendrites: green, lateral horn neurons (LHNs); dark blue, wedge projection neurons (WEDPNs); orange, dopaminergic neurons of the mushroom body (DANs); brown, descending neurons to the ventral nervous system (DNs); pink, lateral horn centrifugal neurons (LHCENTs). The starburst plot also includes some neurons connected only or mainly at their axons, including a small number of: light blue, visual projection neurons; yellow, severed contralateral axons; dark green, putative gustatory projection neurons from the gnathal ganglia; yellow, putative axons ascending from the ventral nervous system. B Schematic illustrating the definitions used to group neurons into broad classes. For details see Methods. C Jitter plot showing olfactory layers of TOONs broken down by predicted transmitter (if known) and broad class (LHONs, LH output neuron; LHLN, LH local neuron) (Frechter et al., 2019). D The percentage of input supplied onto third-order neurons by different classes of input neuron. Upper, inputs onto third-order neurons' dendrite, lower, fourth-order neurons dendrites. Insets, input onto axons. E Normalised synaptic input to layer three and four neurons, as well as LH centrifugal neurons whose dendrites lie outside the LH but whose axons innervate it. Synaptic input is normalised by the total number of input synapses to the neuron's predicted axon or dendrite.

Figure 8-Figure supplement 1. Defining cell types for third-order olfactory neurons.

Figure 8-Figure supplement 2. Split-GAL4 lines for excitatory lateral horn output neurons.

Figure 8-Figure supplement 3. Split-GAL4 lines for inhibitory lateral horn output neurons.

Figure 8-Figure supplement 4. Split-GAL4 lines for lateral horn local neurons.

Figure 8-Figure supplement 5. Split-GAL4 lines for lateral horn input neurons. 
currently have more extant data in the literature e.g. allowing sparse genetic driver lines to be identified, or assignment of developmental identities and putative transmitter expression.

With the benefit of a full, high-resolution LHN inventory from the hemibrain, we re-assessed sparse genetic driver lines we previously generated to help experimentally target specific LHN cell types (Figure 8-Figure Supplement 2,Figure 8-Figure Supplement 3,Figure 8-Figure Supplement 4,Figure 8-Figure Supplement 5). We then grouped neurons into developmentally related 'hemilineages' and assigned all members of a given hemilineage the same 'transmitter identity' if we knew that at least one member of that hemilineage to express acetylcholine, GABA or glutamate based on immunohistochemical work (Dolan et al., 2019). Our assignments (Figure 8C and Figure 8-Figure Supplement 1B) are based on an assumption that neurons of a hemilineage share the same transmitter expression, as has been demonstrated for the ventral nervous system (Lacin et al., 2019). This is a useful proxy that gives an impression of fast-acting neurotransmitter expression diversity throughout the pool of TOONs, but it is far from definitive. We anticipate that machine learning methods will assist in automatic transmitter type classification for synapses in data sets such as the hemibrain in the near future (Eckstein et al., 2020). LHNs are very diverse in terms of their hemilineage origins: $\sim 30 \%$ of known hemilineages in the midbrain contribute to LHNs, with some more biased to layer 3 or layer 4 LHNs (Figure 8D). This is in contrast to KCs, that arise from a set of only four neuroblasts (Truman and Bate, 1988).

All the LHNs we consider are direct targets of olfactory ALPNs and would therefore historically be considered third-order olfactory neurons. In absence of connectivity data this is a necessary and useful simplification. Using the layers (Figure 2C), we can now for the first time take a more quantitative look at their putative position within the olfactory system. This shows that LHNs populate different layers of the olfactory system because the fraction of direct ALPN input can vary widely (Figure 8C,E).

LHNs in layer 3 are mainly putative GABAergic or glutamatergic neurons based on their developmental origins and therefore likely inhibitory, while layer 4 LHNs are more commonly cholinergic and therefore excitatory ( Figure 8A). It is important to note that the layer 4 LHNs are still direct synaptic partners of ALPNs; their designation as layer 4 is a result of weaker direct connectivity from ALPNs and slightly greater local input from layer 3 and 4 neurons (Figure 8D).

Matching hemibrain neurons to light-level data and partial tracings for neurons from FAFB shows that most 'anatomically' local neurons have a layer closer 3, and output neurons a layer closer to 4 ( Figure $8 \mathrm{C}$ ). The uPNs contribute most strongly and directly to the input budgets of layer 3 and 4 LHNs; in contrast, mPNs could be said to short-circuit the olfactory system, connecting to LHNs of layers 3-6 as well as other TOONs of the superior protocerebrum (Figure 8 and Figure 10).

Individual TOON cell types can sample from a variety of ALPNs (Figure 9), and each type exhibits a relatively unique 'fingerprint' of input connectivity. Comparing the cosine similarity in ALPN $\rightarrow$ target connectivity between ALPN cell types reveals that uPNs and mPNs have very different connectivity profiles (Figure 9-Figure Supplement 1). While a certain amount of structure is present, there is no clear subgrouping of ALPN into subsets that serve as preferred inputs onto distinct target subsets. Thermo/hygrosensory ALPN cell types often exhibit similar connectivity with one another, and their uPNs clusters away from purely olfactory uPNs, however, their targets also commonly receive olfactory input from mPN cell types.

By breaking TOONs and their identified inputs into large classes (Figure 8B,E and Figure 10 Figure Supplement 1), we can see that while direct UPN input to TOONs decreases from layers 3 through 5, mPN innervation remains constant and occurs onto both TOON dendrites and axons. Layer 3 TOONs heavily feedback onto ALPNs by making GABAergic axo-axonic contacts, while layer 4 TOONs feedback to layer 3 by both axo-dendritic and dendro-dendritic contacts.

If we think of obvious outputs of the olfactory system, we might consider dopaminergic neurons of the mushroom body (DANs) or putative pre-motor descending neurons (DNs) that project to the ventral nervous system, help to inform the writing of olfactory memory and the control of 
bioRxiv preprint doi: https://doi.org/10.1101/2020.12.15.401257; this version posted May 14, 2021. The copyright holder for this preprint (which was not certified by peer review) is the author/funder, who has granted bioRxiv a license to display the preprint in perpetuity. It is made available under aCC-BY 4.0 International license.

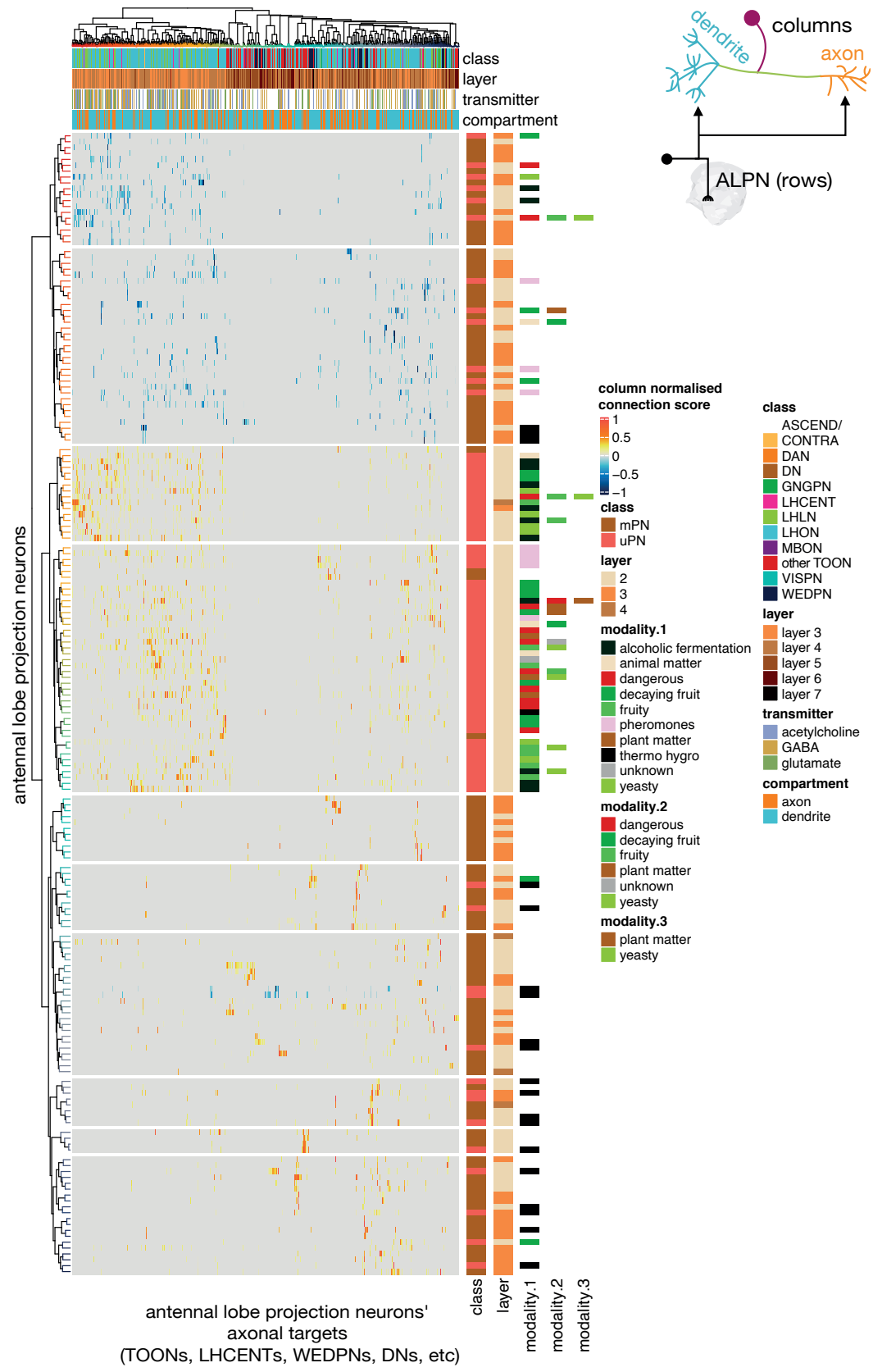

Figure 9. Antennal lobe projection neuron connectivity onto downstream targets. Annotated heatmap showing the ALPN cell types (188, rows) $\rightarrow$ target (column) connection strengths. These connection strengths have been max normalised per column (target). ALPNs known to be glutamatergic or GABAergic have been given negative connection strengths, those that are unknown or cholinergic, positive. Each target column represents an entire connectivity types' dendrites or axons (964 connectivity types' dendrites, 534 connectivity type's axons), in which each neuron has to have at least a 10 synapse or $1 \%$ postsynapse-normalised connection from an ALPN. Annotation bars indicate axons versus dendrites, as well as other metadata. Row and column clusters based on cosine similarity between connection strengths, see Figure 9-Figure supplement 1. Where 'modality' is left white, the cell type in question combines information from multiple antennal lobe glomeruli. Clustering based on Ward's distance, ALPNs grouped into 10 blocks for visualisation.

Figure 9-Figure supplement 1. Neurons at the ALPN axon $\rightarrow$ target connection, clustered by connection similarity 
olfactory-related motor output, respectively. Strong output onto DANs and DNs first occurs with layer 4 TOONs and gets stronger with layer 5 TOONs, these contacts mostly being cholinergic axodendritic ones.

Higher TOON layers receive strong connections from memory-reading output neurons of the $M B$ (MBONs) while lower ones receive greater, putatively inhibitory centrifugal feedback from neurons downstream of MBONs (LHCENTs) (Figure $8 \mathrm{E}$ and Figure 10). Using a neurotransmitter prediction pipeline based on applying machine learning to raw EM data of presynapses in the FAFB data set, LHCENT1-3, LHCENT5-6 and LHCENT9 appear to be GABAergic (Eckstein et alı, 2020). LHCENT4 is predicted to be glutamatergic. LHCENT4 also differs from the others in that it is upstream of most other LHCENTs. LHCENT7 is predicted to be dopaminergic and has also been described as PPL202, a dopaminergic neuron that can sensitise KCs for associative learning (Boto et al., 2019).

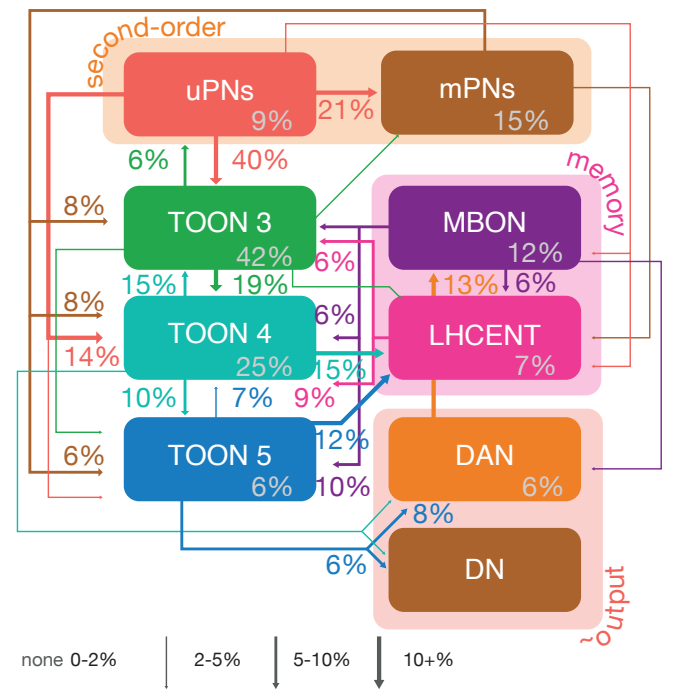

Figure 10. Neuron class-level network diagram of higher olfactory layers. A circuit schematic of third-order olfactory neurons, showing the average connection strength between different classes of neurons (mean percentage of input synapses), broken into their layers, as well as the ALPN, LHCENT and MBON inputs to this system and DAN and DN outputs. The percentage in grey, within coloured lozenges, indicates the mean input that class provides to its own members. The threshold for a connection to be reported here is $5 \%$, and $>2 \%$ for a line to be shown.

Figure 10-Figure supplement 1. Neuron class-level network diagrams of higher olfactory layers, broken down by neuron compartments and putative transmitters.

\section{Stereotypy in superior brain olfactory neurons}

Are these $~ 500$ LHN types reproducible units? To address this question, we looked at the similarity in connectivity among members of the same cell type in the hemibrain data set (Figure 11). We also cross-compared hemibrain neurons with neurons in an EM volume of a different brain (FAFB) (Figure 12A-C) (Zheng et al., 2018). We find that 'sister' uPN - i.e. those that have their dendrites in the same glomerulus and come from the same hemilineage - typically make similar numbers of connections onto common downstream targets. This is especially obvious when targets are grouped by their cell type rather than each considered as individual neurons (Figure 11A-C). Nevertheless, the consistency of these connections differ by sister uPN type, with some (e.g. DM4 VPNs, mean cosine similarity 0.50 ) being less similar to one another than a few non-sister comparisons (e.g. VC1 IPN and VM5V adPN, 0.63) (Figure 11A). For TOON cell types, comparing both up - and downstream connectivity to the axon or dendrite also yields a cosine similarity measure of $\sim 0.75$ (Figure 11-Figure Supplement $1 \mathrm{~A}, \mathrm{~B}$ ), with only a small difference between inputs/outputs and 
axon/dendrites (Figure 11-Figure Supplement 1D,E). The more similar the inputs to a cell type's dendrites, the more similar its axonic outputs (Figure 11-Figure Supplement 1C). Both also correlate with the morphological similarity between TOONs of a cell type (Figure 11-Figure Supplement 1E).

For comparisons with FAFB, we picked 10 larval-born 'secondary' hemilineages in the hemibrain data set and coarsely reconstructed all neurons of the same hemilineages in the FAFB volume (see Methods). We show that the morphologies can be matched between the two data sets and that, visually, these matches can be striking (Figure 12A and Figure 12-Figure Supplement 4A). Every LHN and wedge projection neuron (Bates et al., 2020b) hemibrain cell type in these 10 hemilineages can be matched to one in FAFB (172 cell types), with some small variability in cell number per brain (Figure 12B, Figure 12-Figure Supplement 1). We also examined a set of 'primary' embryonic-born neurons, the LH centrifugal neurons LHCENT1-11, and could match them up well between the two data sets. In some cases, putative cell types that appear isomorphic 'at light-level' can be broken down into several connectivity sub-types.

In several cases, we see that each of these subtypes have small but consistent morphological deviations between the two data sets (Figure 12-Figure Supplement 2A). To account for this, we broke our 569 morphological cell types into 642 connectivity types (Scheffer et al., 2020). In general, the closer the two neurons' morphology, the more similar their connectivity. However, similar morphologies can also have different connectivity (Figure 12-Figure Supplement 4B), perhaps due to non-uniform under-recovery of synapses during the automatic segmentation of neurons and their connections in the hemibrain (Scheffer et al., 2020).

It is difficult to directly compare synapse numbers between the two data sets, as the methods of reconstruction were very different (see Methods). In FAFB, each human-annotated polyadic synapse has a mean of 11 postsynapses, whereas in the hemibrain machine-annotation has resulted in $\sim 8$ (for the same, cross-matched neurons) (Figure 12-Figure Supplement 4D). This is likely because different reconstruction methodologies have resulted in different biases for synaptic annotation. Nevertheless, we aimed to see whether ALPN $\rightarrow$ LHN connections in FAFB were also present in the hemibrain data set.

We previously reconstructed all members of selected cell types in FAFB (Bates et al., 2020b). Here, we manually reviewed the same types in the hemibrain data set (an average of 3 neurons per type) so that they are far more complete than the average hemibrain LHN (Scheffer et al., 2020) (see Methods). We also examined other cell types for which we have only subsets in FAFB (Figure 12Figure Supplement 4A). Normalised connections strengths (normalised by total input synapses) from ALPNs to LHNs are, on average, stronger in the hemibrain than in FAFB. In the hemibrain a larger total number of input synapses have been assigned per neuron but fewer ALPN $\rightarrow$ LHN connections, perhaps an artefact of the different reconstruction methods employed (Figure 12Figure Supplement $4 \mathrm{C}$ ). Nevertheless, by comparing our FAFB reconstructions with their cognates in the hemibrain for 12 connectivity types, using a cosine measure for connection similarity, we see that the variability in ALPN $\rightarrow$ LHN connections between data sets is no greater than within the same data set (Figure 12C and Figure 12-Figure Supplement 2B).

This suggests that morphological cell types may be as consistent between animals as within an animal. We also compare the hemibrain connectivity to a data set describing functional connectivity between antennal lobe glomeruli and LHNs (Jeanne et al., 2018). For some LHNs these functional connections are well recapitulated in the hemibrain's cognate UPN $\rightarrow$ LHN synaptic connectivity. For many other pairs, however, the connectivity similarity is no greater than that to other neurons in the data set (Figure 12D and Figure 12-Figure Supplement 3): some functional connections are not present as direct synaptic connections in the connectome and vice versa. Similarly, there is no clear correlation between the strength of a functional connection and the synaptic strength of corresponding hemibrain ALPN $\rightarrow$ LHN connections (Figure 12-Figure Supplement 3D,E). This could be due to the action of local processing in the LH as well as connections from mPNs, which have impacted feed-forward transmission more for some LHN cell types than for others. For example, LHAV4a4 neurons have very similar structural and functional connectivity, while LHAV6a1 neurons 
bioRxiv preprint doi: https://doi.org/10.1101/2020.12.15.401257; this version posted May 14, 2021. The copyright holder for this preprint (which was not certified by peer review) is the author/funder, who has granted bioRxiv a license to display the preprint in perpetuity. It is made available under aCC-BY 4.0 International license.
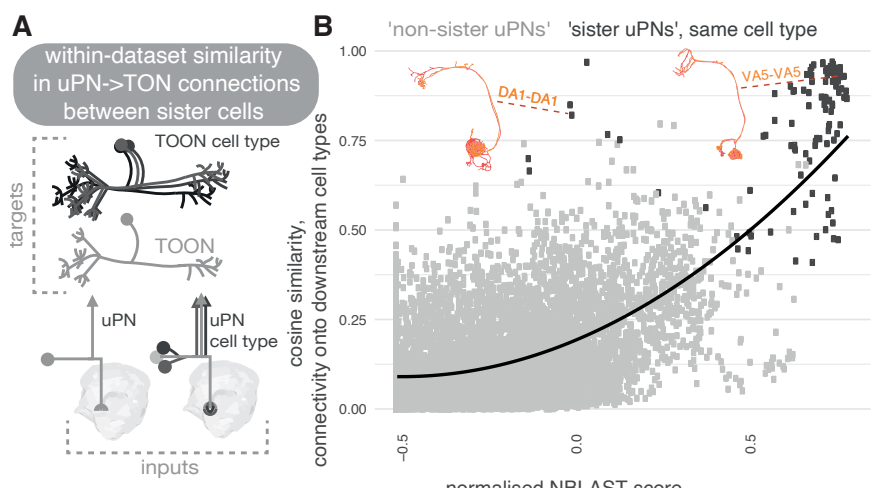

normalised NBLAST score morphological similarity

D

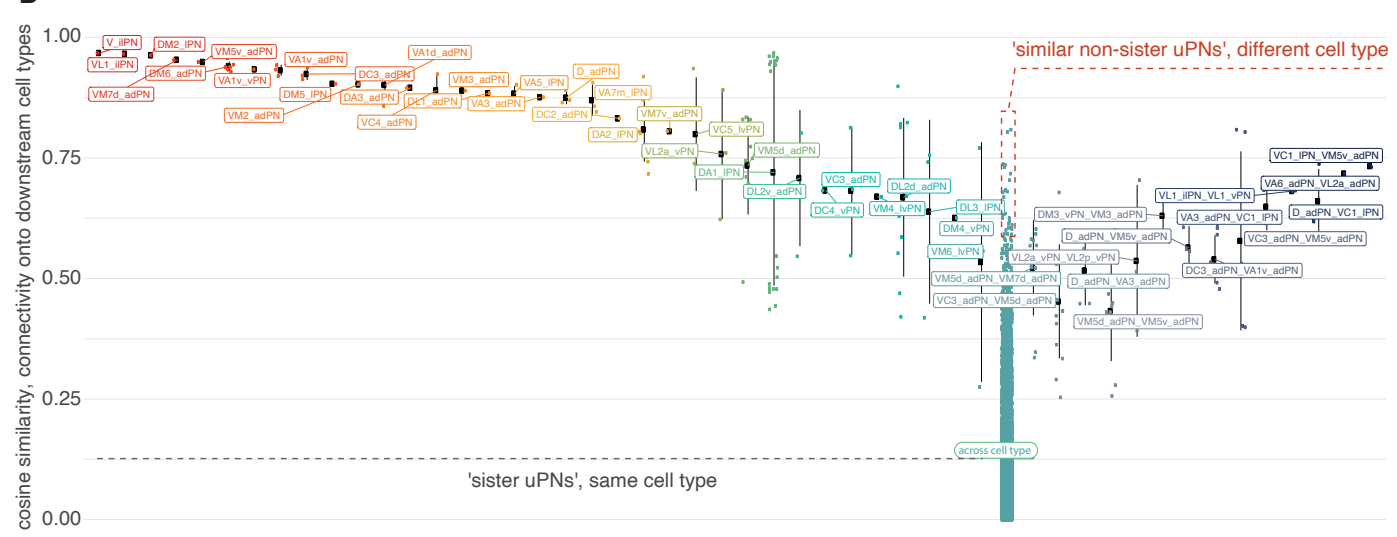

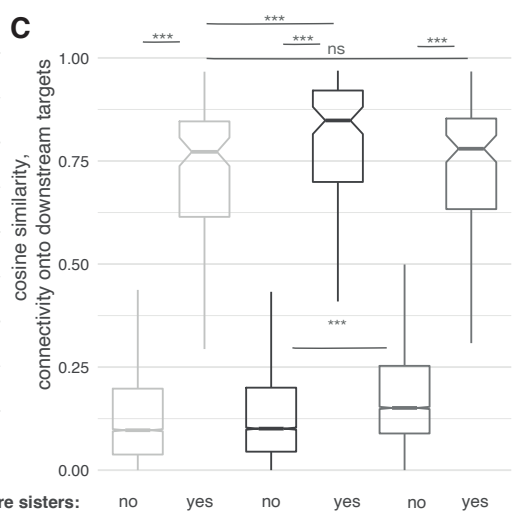

uPNs are sisters: TOON targets are:

single single yes no yes non-

Figure 11. Within-data set connectivity similarity for key olfactory cell types. A The synaptic targets of uPNs (left) and uPN cell types (right) can be thought of as both individual downstream cells (lower) as well as cell types (upper). B For each pair of uPNs, the cosine similarity for their outputs onto downstream cell types is compared against their morphological similarity. The UPN-uPN pairs where both neurons are from the same cell type, 'sisters', shown in dark grey, otherwise in light grey. C The cosine similarity in the downstream target pool for sister and non-sister uPN pairs is compared. Targets can either be considered as separate cells (light grey, leftmost boxplots) or pooled by cell type (dark grey, middle boxplots). Shuffled data, for which cell type labels were shuffled for neurons downstream of each UPN to produce random small out-of-cell-type groupings of cells, shown in mid grey (rightmost box plots). Non-sister TOONs are shuffled pairs of TOONs from different cell types. There are 113 different sister PN-PN comparisons, and 9157 non-sister PN-PN comparisons, from our pool of 136 uniglomerular PNs. D The cosine similarity between connections to downstream cell. Left, all reconstructed LHNs types, for UPN-uPN pairs. Pairs shown are from the same cell type (left) or different cell types, where at least one comparison has a similarity of above $>0.6$. Significance values, Wilcoxon test: $* * *: p<=0.001$.

Figure 11-Figure supplement 1. Similarity in connectivity up and downstream of olfactory neurons. 
do not, though both their structural and functional connectivity seem stereotyped even if they are different from one another (Jeanne et al., 2018; Fişek and Wilson, 2014). In addition, functional connection strength integrates inhibitory and excitatory inputs from different ALPN classes, which might also confound our results. Indeed, the glomeruli for which we have some of the largest deviations from the hemibrain structural data are those with GABAergic uPNs (Figure 12-Figure supplement 3B).

\section{Integration of innate and learned olfactory pathways}

With the hemibrain data set, we can look at the extent to which MBONs directly connect to LHNs. We see that while most olfactory ALPN input is onto LHN dendrites, most MBON input is onto their axons (Figure 13A,C,D). We quantify this using an ALPN-MBON axon-dendrite compartment separation score (see Methods) and find high compartmental segregation of inputs, with MBONs inputting onto LHN axons (though many cells have a score at or near zero as they receive little MBON innervation) (Figure 13-Figure Supplement 4). Many of those with negative scores are either neurons tangential to the $\mathrm{LH}$ or $\mathrm{LH}$ centrifugal neurons, whose MBON innervation is known to target their dendrites (Bates et al., 2020b). More than $20 \%$ of layer four LHN axons are targeted by a range of MBONs (Figure 13C): both cholinergic and GABAergic, and including MBONs implicated in both aversive and appetitive learning (Aso et al., 2014b). MBON connectivity to LHNs is sparse and only a few LHNs receive inputs from multiple MBONs (Figure 13E,F). With MBON $\rightarrow$ LHN connections being axo-axonic, there is the potential of them being reciprocal. However, there is very little output from LHNs onto MBON axons (Figure 13B), suggesting that MBONs might gate LHN activity, but not vice versa.

Next, we asked whether MBONs target the axons of LHNs that pool particular kinds of olfactory information. To examine this question, we performed a matrix multiplication between connectivity matrices for ALPN $\rightarrow$ LHN dendrite innervation, and MBON $\rightarrow$ LHN axon innervation, normalised by the LHN compartment's input synapse count, to generate a 'co-connectivity' score (Figure 13Figure Supplement 1C-D). From this, three coarse groups emerge: some MBON types seem to preferentially target 'putative food related' LHNs. These LHNs receive input from ALPNs that respond to mostly yeasty, fruity, plant matter and alcoholic fermentation-related odours. Another group preferably targets a separate set of LHNs, that themselves receive input from ALPNs involved in thermosensation, ethanol, $\mathrm{CO}_{2}$, aversive fruity odours and pheromones. The third pool of MBONs wire with neurons from both pools of ALPNs. About half the uPNs did not have a strong coconnectivity score with MBONs. To try and assess whether certain MBONs might play a role in the processing of particular odours, we multiplied the co-connectivity matrix by odour response data from a recent study (Badel et al., 2016). We did not see a striking separation, though all MBONs converge on TOONs that get appetitive fruity odours (e.g. ethyl butyrate) information from PNs, largely because these odours are well represented on the PN level, and less so highly specific odours that are less broadly encoded (Figure 13-Figure Supplement 1A), such as the bacterial odour geosmin.

In examining neurons downstream of MBONs, we found a cell type of 12 neurons which receives an unusually high proportion, up to $\sim 37 \%$, of their input connections from MBONs: LHAD1b2, cholinergic LH output neurons whose activation generates approach behaviour (Dolan et al., 2019; Frechter et al., 2019). Electrophysiological recording of these cells has shown them to act as a categoriser for 'rotting', amine-type odours (Frechter et al., 2019). Consistent with connectivity observed in FAFB (Bates et al., 2020b), we find now the full suite of excitatory, naively aversive and inhibitory appetitive MBONs that target LHAD1b2 axons, and the naively appetitive MBONs and specific ALPNs that target their dendrites (Figure 13-Figure Supplement 2A,B). We also observe LHAD1 b2 connections onto the dendrites of PAM DANs involved in appetitive learning, again consistent with work in FAFB (Otto et al., 2020) (Figure 13-Figure Supplement 2C). Together, this builds a model whereby naively appetitive information from the LH signals the presence of rotting 
bioRxiv preprint doi: https://doi.org/10.1101/2020.12.15.401257; this version posted May 14, 2021. The copyright holder for this preprint (which was not certified by peer review) is the author/funder, who has granted bioRxiv a license to display the preprint in perpetuity. It is made available under aCC-BY 4.0 International license.
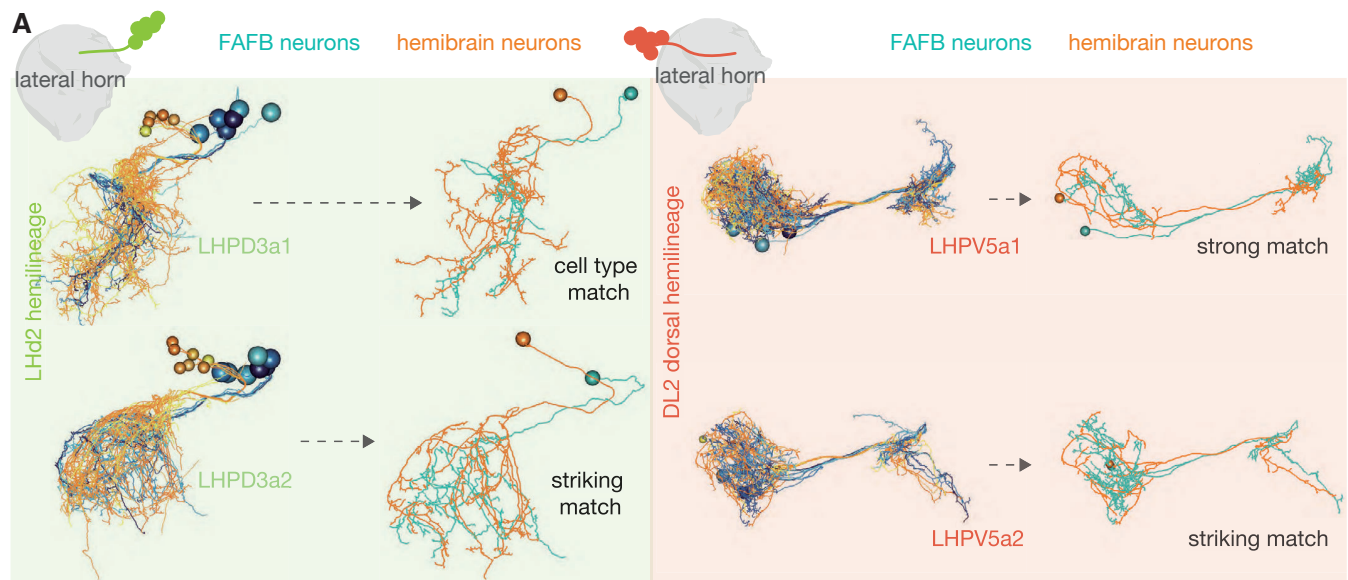

B
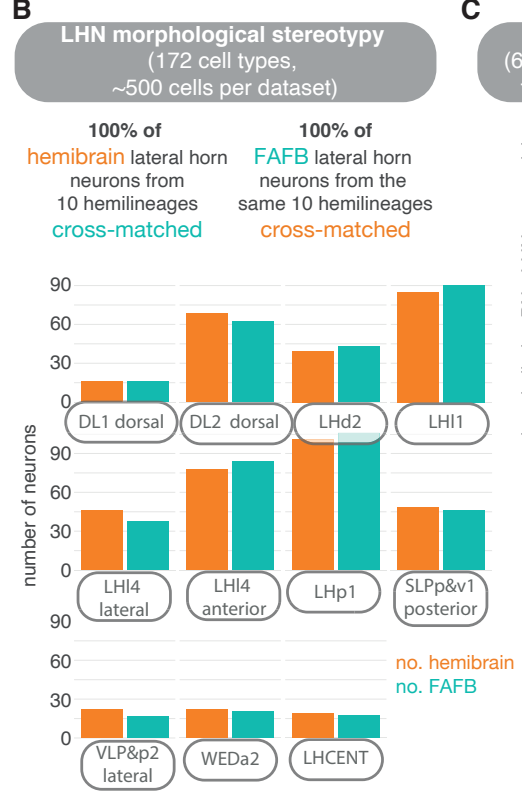

C

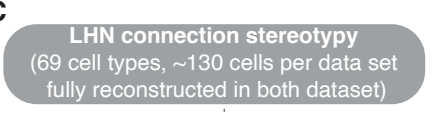

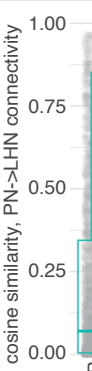
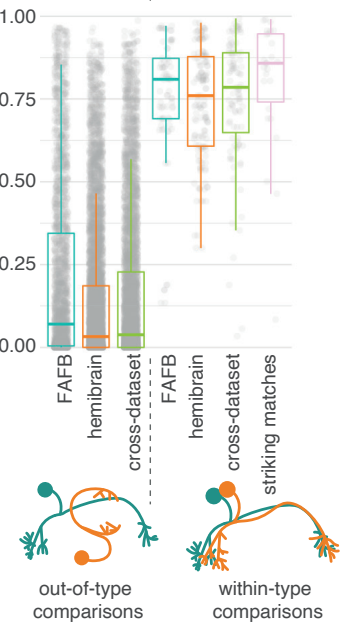

D

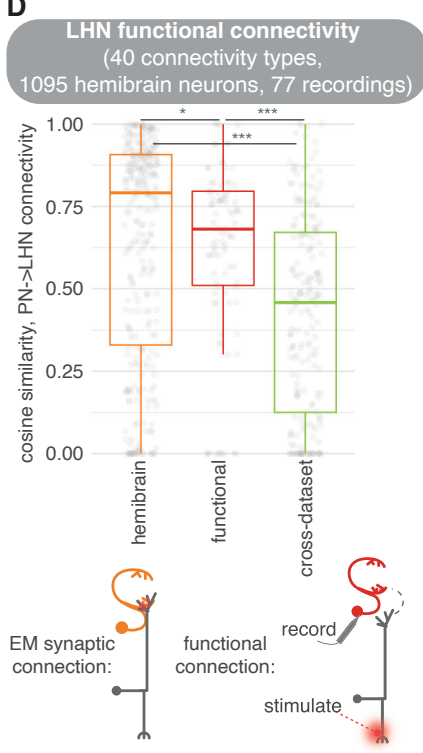

Figure 12. Stereotypy in morphology and connectivity between lateral horn neurons in the hemibrain, FAFB and functional data sets. A Cell types and individual neurons that have been cross-matched between data sets. Examples from the hemilineages LHd2 (i.e. the dorsal most cell body group in the LHd2 lineage clone, otherwise known as DPLm2 dorsal) and DL2 dorsal (otherwise known as CP3 dorsal). B We were able to cross-match $>600$ neurons across 10 hemilineages between the hemibrain and FAFB. C For neurons that had been fully synaptically reconstructed in FAFB, we calculate the cosine similarity for their ALPN $\rightarrow$ LHN connectivity vectors to hemibrain neurons, both out-of-cell-type (left) and within-cell-type (right), as well as between the two data sets. In pink, same-cell-type between data set comparisons are made for only our 'best' morphological matches; matches for which the two neurons look so similar they could be the 'same cell'. D Within-cell-type cosine similarity for ALPN $\rightarrow$ LHN connectivity for within the hemibrain data set, within the Jeanne et al. (2018) functional connectivity data set, and between members of the same cell type across data sets. Significance values, Student's T-test: ns: $\mathrm{p}>0.05 ; *: \mathrm{p}<=0.05 ; * *: \mathrm{p}<=0.01 ; * * *: \mathrm{p}<=0.001 ; * * * *: \mathrm{p}<=$ 0.0001

Figure 12-Figure supplement 1. Stereotypy in morphology between lateral horn neurons in the hemibrain and FAFB data sets.

Figure 12-Figure supplement 2. Stereotypy in connectivity between lateral horn neurons in the hemibrain and FAFB.

Figure 12-Figure supplement 3. Stereotypy in connectivity between lateral horn neurons in the hemibrain and a functional data set.

Figure 12-Figure supplement 4. Matching synaptically complete neurons between two EM data sets. 
bioRxiv preprint doi: https://doi.org/10.1101/2020.12.15.401257; this version posted May 14, 2021. The copyright holder for this preprint (which was not certified by peer review) is the author/funder, who has granted bioRxiv a license to display the preprint in perpetuity. It is made available under aCC-BY 4.0 International license.
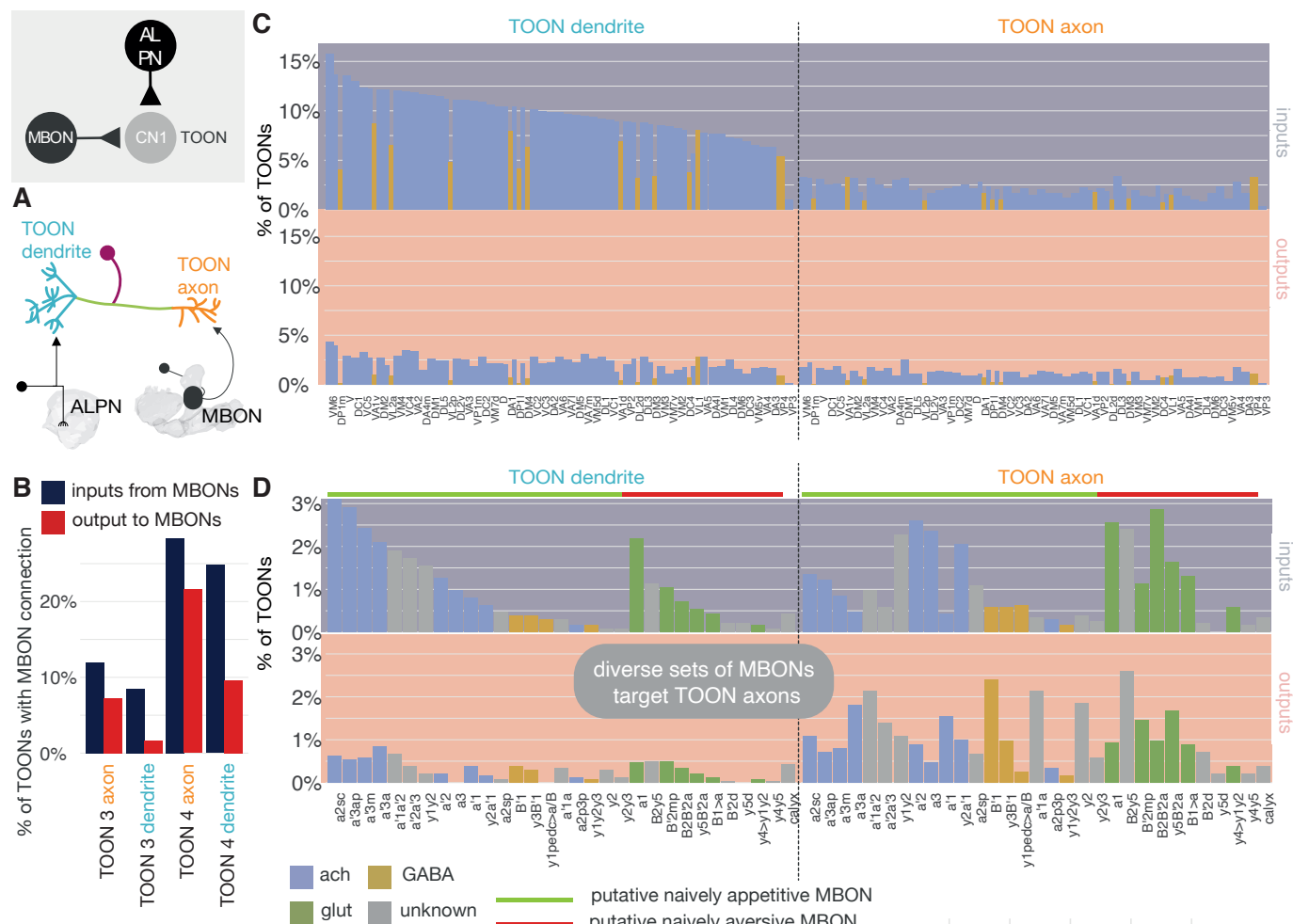

E

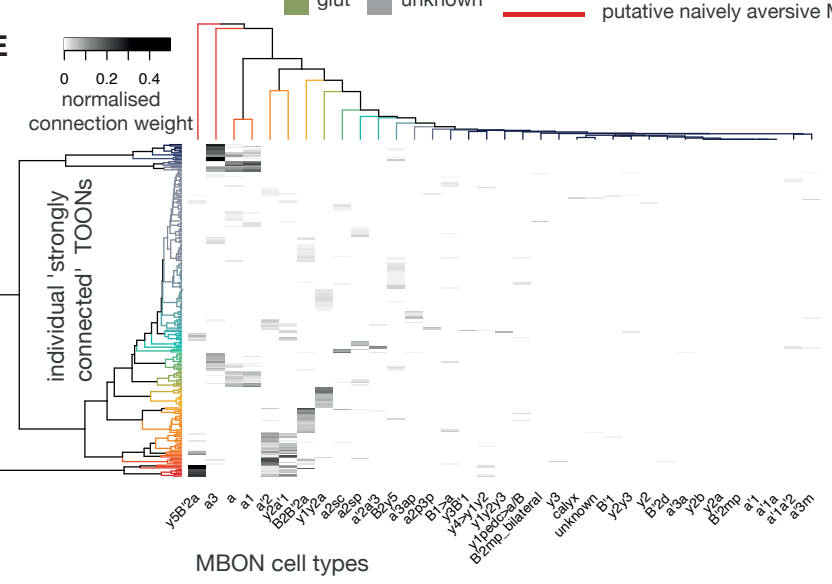

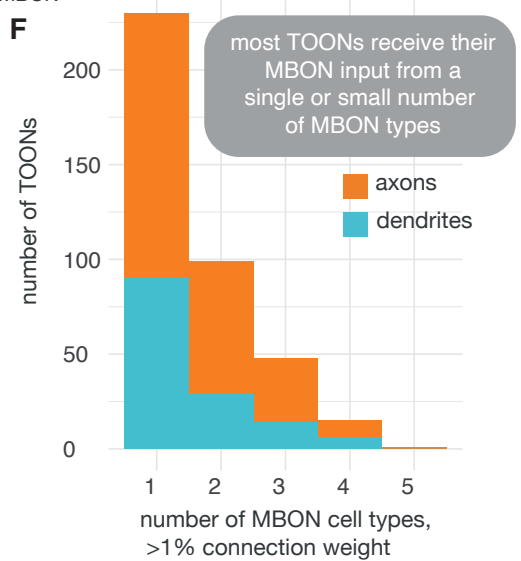

Figure 13. MBON innervation of lateral horn neurons. A Olfactory projection neurons and MBONs seem to target different ends of lateral horn output neurons. B The percentage of TOONs (2383 neurons in total) that receive a 'strong' connection from an MBON type (71 neurons in total) ( $>1 \%$ of their dendrite's/axon's input synapses). C Percentages are broken down by MBON cell type. D The percentage of TOONs that receive a 'strong' connection from a uPN type (136 neurons in total), broken down by type ( $>1 \%$ of their dendrite's/axon's input synapses). E A heatmap showing the normalised input of different MBONs onto TOONs' axons. F A histogram showing the number of downstream TOONs that receive input from different numbers of MBONs. A threshold of $>1 \%$ the input synapse count is used, axons and dendrites treated separately.

Figure 13-Figure supplement 1. Propagating known odour information to third-order olfactory neurons and mushroom body output neurons.

Figure 13-Figure supplement 2. An exemplar convergence cell type of the lateral horn and mushroom body.

Figure 13-Figure supplement 3. Convergence neurons of the lateral horn and mushroom body.

Figure 13-Figure supplement 4. A class-compartment separation score. 
fruit (Mansourian and Stensmyr, 2015). This activity is then bidirectionally gated by MBON input: expression of an aversive memory reduces the cholinergic drive to the axon, while an appetitive memory reduces glutamatergic inhibition, thereby potentiating the cell type's effect on its downstream targets. If the cell type fires, it could excite PAM DANs that feedback to create a long-term depression in MB compartments associated with naive aversion, i.e. appetitive learning.

The next level at which 'innate' information from the non-MB arm of the olfactory system and 'learned' information from the MB arm can converge, is in 'convergence' neurons (CN2) downstream of both of these neuropils. By looking at LHN cell types known to evoke either aversive or appetitive behaviour (Figure 13-Figure Supplement 3A) (Dolan et al., 2019), we see that downstream partners of appetitive LHNs are more likely to be innervated by MBONs than those of aversive LHNs (Figure 13-Figure Supplement 3C). CN2 neurons that receive at least $1 \%$ of their synaptic inputs from LHNs or from MBONs tend to get cholinergic input from naively appetitive MBONs and LHNs, and inhibitory input from naively aversive MBONs and LHNs (Figure 13-Figure Supplement 3B,D).

\section{Connections to the motor system}

Motor systems ultimately responsible for generating behaviour are located in the ventral nervous system and the suboesophageal zone (SEZ) and can, to some extent, function independently of the rest of brain (Berni et al., 2012; Hückesfeld et al., 2015; Egeth, 2011; Hampel et al., 2017). How olfactory circuits connect to and modulate these motor systems remains an open question. In general, higher brain circuits exert control over motor systems via descending neurons (DNs) (Lemon, 2008). In Drosophila, a recent light-level study identified $\sim 700$ DNs ( 350 per side of the brain) that connect the brain to the ventral nervous system (Namiki et al., 2018). We used existing neuPrint annotations and complemented them with DNs identified in the "FlyWire" segmentation of FAFB to compile a list of 345 confirmed DNs in the hemibrain data set (see supplemental files) (Dorkenwald et alo, 2020). Due to the truncation, the hemibrain volume does not contain many of the DNs in the SEZ ("DNg" in Namiki et al., 2018) and most of the DNs present descend from higher brain regions. Even without knowing their exact targets in the ventral nervous system, such DNs represent a common outlet for all higher brain circuits. We find only 11 DNs that appear to be "early" (i.e. layer 3 or 4) with respect to the olfactory system (Figure 14A,B). These early DNs typically receive diverse inputs including from ALPNs and lateral horn neurons (LHNs) (Figure 14C). We next asked whether individual DNs exhibit preferences with respect to which types of antennal lobe receptor neurons (ALRNs) they receive direct or indirect input from. To answer this, we re-ran the graph traversal model using only the ALRNs of a given type/glomerulus as seeds. This produced, for each DN, a vector describing the distances to 49 different ALRN types (we excluded some of the more severely truncated glomeruli). Using those vectors to calculate the lifetime kurtosis, we find both broad and sparse early DNs (Figure 14D). By contrast, DNs in layer 5 and above are generally broadly tuned and no longer exhibit a preference for specific ALRNs (data not shown). There do appear to be "shortcuts" between the thermo/hygrosensory and the motor system via early DNs that connect most directly to VP1-5 ALRNs. One might expect similar connections for biologically highly relevant odours such as the wasp pheromone Iridomyrmecin (Or49a/Or85f, DL4), Geosmin (Or56a, DA2) or the sex pherhomone cVA (Or67d, DA1) (Mansourian and Stensmyr, 2015; Stensmyr et al., 2012; Kurtovic et al., 2007; Kohl et al., 2013; Ebrahim et al., 2015). However, ORNs appear to only converge onto broadly tuned early DNs that show no clear preferences for specific odour scenes (Figure 14E and Figure 14-Figure Supplement 1). This suggests that thermo/hygrosensation employs labeled-line shortcuts whereas olfaction uses (higher-order) population coding to effect motor output.

\section{Discussion}

One of the most significant practical outcomes of our work are classifications for thousands of olfactory system neurons across the hemibrain data set, comprising a full inventory for a single brain 
bioRxiv preprint doi: https://doi.org/10.1101/2020.12.15.401257; this version posted May 14, 2021. The copyright holder for this preprint (which was not certified by peer review) is the author/funder, who has granted bioRxiv a license to display the preprint in perpetuity. It is made available under aCC-BY 4.0 International license.

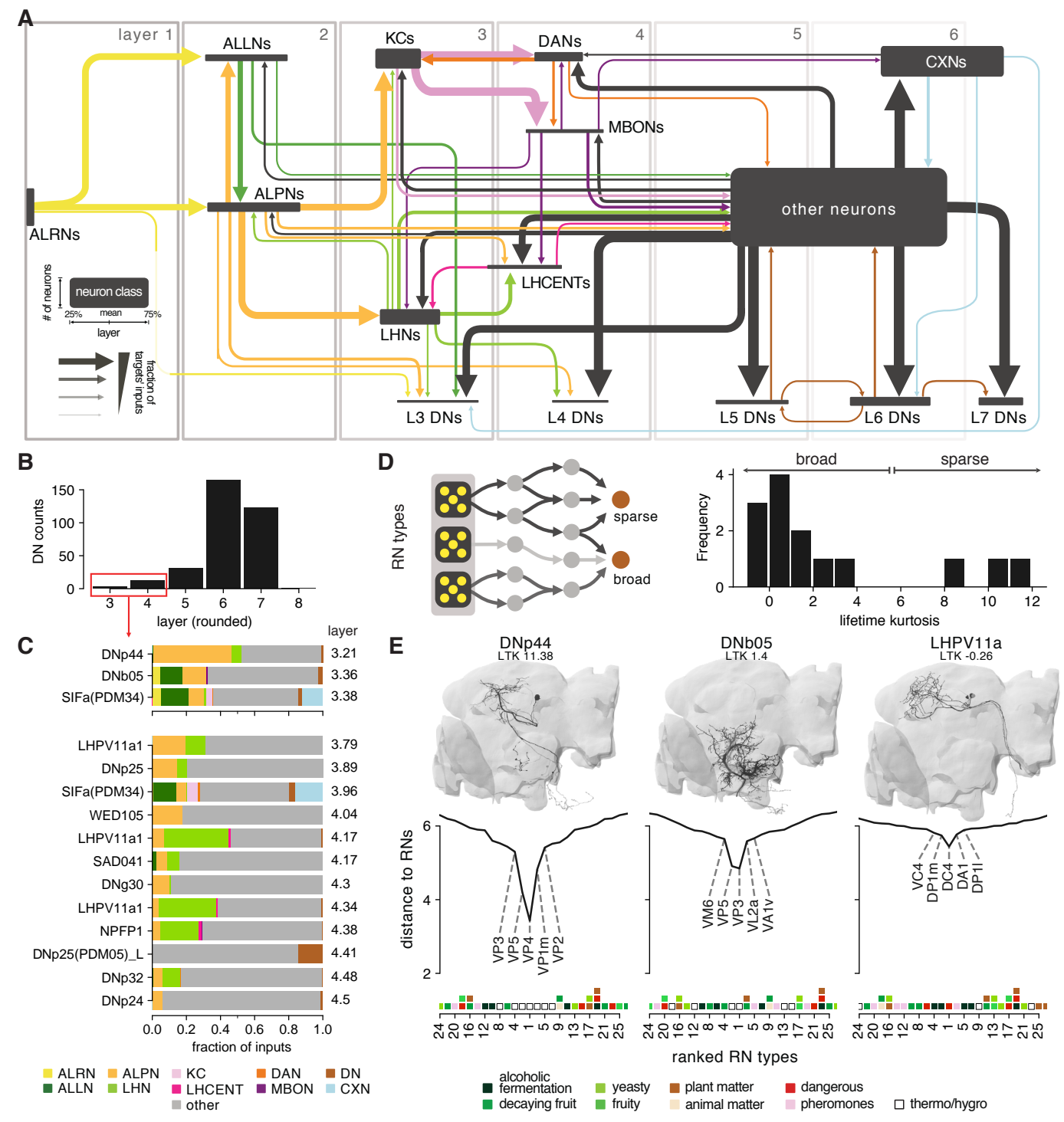

Figure 14. Connections between the olfactory system and descending neurons. A Summary of olfactory circuits organised by layers. Box heights and widths correspond to the number and layer of neurons represented, respectively; arrow widths correspond to fraction of the targets' inputs. See also legend in lower left. B The number of "early" (layer 3 and 4) descending neurons (DNs) is low. C Inputs to early DNs are diverse. Labels represent names in neuPrint. D Sparseness (lifetime kurtosis, LTK) of early DNs with respect to individual receptor neuron (ALRN) types. Most early DNs receive indirect inputs from a broad range of ALRNs. E Exemplary DNs and their connectivity to individual ALRN types. A low distance indicates a more direct connection between an ORNs or TRN/HRN type and the DN. Only the top 25 ALRN types shown. Hemibrain DNs are shown in black and their homologs in the FlyWire dataset as reference in grey. Heatmap shows glomeruli odour scenes.

Figure 14-Figure supplement 1. Extended data for Figure 14E.

hemisphere (see Supplemental Material). This includes the first full survey of antennal lobe local neurons (ALLNs), third-order olfactory neurons (TOONs) and lateral horn centrifugal neurons (LHCENTs), and complements a recent inventory of antennal lobe projection neurons (ALPNs) (Bates et al., 2020b) (Figure 1). We explore this data with a model that breaks down the olfactory system into layers. Layering had not previously been computable for higher-order neurons, and this analysis reveals interesting features even within the first three layers. Additionally, we have investigated high-level connectivity motifs between the neuron classes and cell types that we have defined and examined how stable our classifications are by asking whether we can find the same neurons, and 
in some cases the same connections, in a second connectomic data set.

\section{Cell type annotations across the first three orders of the olfactory system}

We have built open-source neuroinformatic tools in R and Python (see Methods) to read and summarise neuron data from the hemibrain data set efficiently. We have used these with morphological clustering tools, namely NBLAST (Costa et al., 2016), to break neurons into groups that we can validate against other neuron data, both from light microscopy (Chiang et al., 2011) and another EM data set (Zheng et al., 2018). In so doing, for the right hemisphere, we have classified all 2644 receptor neurons (ALRN, olfactory and thermo/hygrosensory) in all 58 antennal lobe (AL) glomeruli, as well as the 338 second-order projection neurons (uPNs and mPNs) and 196 antennal lobe local neurons (ALLNs), and 2300 third-order neurons outside of the mushroom body. We connect these olfactory neurons to known cell types, and for ALLNs (Figure Figure 6E) and lateral horn neurons (LHNs) we have expanded extant naming systems to cover hundreds of new morphologies (Figure Figure $8 \mathrm{~A}$ ). For the whole hemibrain data set of $\sim 25,000$ neurons we assign a putative olfactory layer (Figure Figure 2). We find that for layers 1-3, information is mostly propagated forward, for layers 4-6 there is much intra-layer cross-talk, and from 7 onwards information tends to propagate back to lower layers (Figure Figure 2G). In light of this new data, we have also re-evaluated the neurons targeted by recently published lateral horn split-GAL4 lines (Dolan et al., 2019) (Figure 8-Figure Supplement 2, Figure 8-Figure Supplement 3, Figure 8-Figure Supplement 4, Figure 8-Figure Supplement 5).

\section{Class-level connection motifs in the olfactory system}

We have found that connectivity with respect to first-order olfactory inputs, the ALRNs, differs depending on whether the axon enters the antennal lobe from the ipsi - or contralateral side of the brain (Figure 3). Although there have been functional indications of asymmetric information processing (Gaudry et al., 2013) no connectomic signature had been observed in adult Drosophila before, while in larva ORNs are unilateral. We identify a general principle that ipsilateral sensory input has stronger feedforward connections to the ALPNs that convey information to higher centres, while contralateral ALRNs are biased to form connections with antennal lobe local neurons. We also show specific connectivity motifs such as the extreme bias for contralateral sensory input of the broadly innervating bilateral il3LN6 neurons, which appear to be the adult analogue of the larval 'Keystone' (Berck et al., 2016) ALLNs (Figure Figure 5B,E). We see that many sparse ALLNs innervating a small number of glomeruli interact specifically with thermo/hygrosensory circuits; although this is consistent with a model in which these 7 glomeruli form a specialised subsystem, there are local interactions with other glomeruli so they are not completely isolated. Furthermore, some ALLN cell types are segregated into axon and dendrite, which facilitates reciprocal interactions between, for example, the 'heating' glomerulus VP2 and the 'cooling' glomerulus VP3. The antennal lobe also receives feedback from superior brain regions and this primarily targets the ALLN network, as opposed to ALPN dendrites or ORN axons (Figure Figure 5G).

Amongst ALPNs, we see a second general rule: while uPNs mostly receive feedforward input, multiglomerular mPNs get a higher proportion of their input from lateral ALPN-ALPN connections and from ALLNs, meaning that in our analysis many emerge as layer 3 neurons (Figure Figure 2E,F). The uPNs provide most of the feedforward drive to the third-order olfactory neurons (TOONs). However, they provide decreasing levels of input to TOONs from layer 3 to layer 5 . They receive feedback to their axons from largely glutamatergic or GABAergic layer 3 TOONs (cells we once classed as LH local neurons) and LH centrifugal neurons. We expect these connections to inhibit UPN axons. The mPNs can short-circuit this progression, and provide roughly consistent amounts of input to all groups of TOONs, both at their dendrite and axons. Comparison with our recent work reveals that we had previously thought of layer 3 TOONs as 'local' neurons and layer 4+ LHNs as 'output' neurons (Figure Figure $8 \mathrm{E}$ ). As olfactory information filters through to layer 5+ TOONs, 
stronger connections are made to 'outputs' of the olfactory system, including dopaminergic neurons that can inform memory and descending neurons that contact premotor circuits (Figure Figure 10-Figure Supplement 1).

These output neurons can get strong but sparse input from a diversity of MBONs to their axons, acting as 'convergence level 1' (CN1) neurons that re-connect the non-MB and MB arms of the olfactory system (Figure Figure 13A-F). This MBON innervation is biased towards TOONs that receive input from certain ALPN groups, including those that encode food-like odours (Figure Figure 13G). Neurons downstream of TOONs can also receive MBON input; these are 'convergence level 2' (CN2) neurons. There are more CN2 neurons downstream of known appetitive TOONs than aversive ones (Figure Figure 13-Figure Supplement 1F). In general, CN2 neurons tend to get inhibitory inputs from naively aversive MBONs and TOONs, and excitatory input from naively appetitive MBONs and TOONs (Figure 13-Figure Supplement 3). Analogous innate-learned integration has been studied in the larva, also in connectome-informed experimentation (Eschbach et alo, 2020). The authors investigated a CN2 cell type and found it to be excited by appetitive LHNs and MBONs and inhibited by aversive MBONs. Naive MBON activity is likely to be relatively stereotyped between animals (Mittal et al., 2020). The hypothesis is that in naive animals, opposing MBON drive balances to produce a stereotyped 'innate' outcome; learning then shifts this balance to bias behaviour.

\section{Between-animal stereotypy in olfactory system neurons}

One of the most pressing questions for the field now is how stereotyped the fly brain actually is. This is critical for interpreting connectomes, but also a fundamental issue of biology across species all the way to mammals. We do not expect two fly connectomes to be exactly the same. However there is a palpable expectation that one would identify the same strong partners for a neuron of experimental interest or reveal a shared architecture of some circuit because many small cell types are faithfully reproduced between animals (Bates et al., 2019).

Here, we have found that all ALPN cell types from a complete survey in FAFB could be found in the hemibrain, with small variations in cell number that correlate with birth-order (Figure 6C and Figure 6-Figure Supplement 1A). More variation occurs in the number of larval-born secondary neurons than the primary neurons born in the embryo. There are several possible reasons for these differences, including the fact that in the larva, each of 21 olfactory glomeruli is defined by a single ORN and ALPN. Since missing one neuron would therefore eliminate a whole olfactory channel, there might be a strong drive to ensure numerical consistency.

Assessing cell type stereotypy of mPNs and ALLNs between hemibrain and FAFB is somewhat compromised by truncation of glomeruli in the hemibrain data set. However, examining morphologically far more diverse LHNs, we could find the same cell types across 10 hemilineages in similar numbers (Figure Figure 12-Figure Supplement 1).

Because LHNs also have reasonably stereotyped dendritic projections (Dolan et al., 2019), functional connections from ALPNs (Jeanne et al., 2018) and responses to odorants (Frechter et al., 2019), it is likely that ALPN-LHN contacts have intrinsic relevance to the animal. Conversely, olfactory ALPN-KC contacts have minimal intrinsic meaning and exhibit near-random wiring (Eichler et al., 2017; Zheng et al., 2020; Caron et al., 2013) although connection biases may enable associative memory to focus on certain parts of olfactory space (Zheng et al., 2020). ALPN connectivity onto third-order neurons in the 'non-MB' path through the olfactory system appears to be reasonably stereotyped, as suggested by the strong morphological stereotypy among these higher-order neurons (Figure Figure 12). Structural connectivity from the hemibrain does not necessarily capture functional connections assayed by physiology. Encouragingly, however, recent work with a retrograde genetic system for finding neurons that input onto genetically targetable cells found 6/7 glomerular connections to LHPD2a1/b1 neurons of above 10 synapses in FAFB, and 8/9 for LHAV1a1 (Cachero et al., 2020). 
735

736

737

738

739

740

741

742

743

744

745

746

747

748

749

750

751

752

753

754

755

756

757

758

759

760

761

762

763

764

765

766

767

768

769

770

771

772

773

774

775

776

777

778

779

780

781

782

\section{Conclusion}

Our study (together with the work of Li et al. (2020) on the mushroom body) provides an annotated guide to the complete olfactory system of the adult fly. We believe that it will be invaluable in driving future work in this important model system for development, information processing and behaviour. Our microcircuit analysis already raised specific hypotheses about brain functions including stereo processing of odours, higher order feedback controlling sensory processing and the logic of integration downstream of the two main higher olfactory centres.

The tools and analytic strategies that we have developed should enable many future analyses of the hemibrain dataset as well as in progress and planned datasets for the male and female central nervous system. For example the layer analysis could usefully be carried out across sensory modalities to quantify multisensory integration. They also provide a quantitative basis for comparative connectomics studies across datasets, for which we provide initial comparisons at two different levels of the olfactory system. Finally, these strategies and the circuit principles that they uncover provide a platform for connectomics approaches to larger brains that will surely follow (Abbott et al., 2020).

\section{Acknowledgments}

This work was supported by a Wellcome Trust Collaborative Award (203261/Z/16/Z) to G.S.X.E.J., and G.M.R.; an ERC Consolidator grant (649111) and core support from the MRC (MC-U105188491) to G.S.X.E.J.; NIH BRAIN Initiative grant 1RF1 MH120679-01 to Davi Bock and G.S.X.E.J.; a Boehringer Ingelheim Fonds PhD Fellowship and a Herchel Smith Studentship to A.S.B.; NIH R01DC008174 (to Rachel Wilson) and an F31 fellowship (DC016196) to A.B.-M; and by the Howard Hughes Medical Institute. We thank the FlyEM team and their collaborators for pre-publication access to the hemibrain data set and reconstructions. Development and administration of the FAFB tracing environment, analysis tools and G.S.X.E.J, T.S. and P.S. were funded in part by NIH BRAIN Initiative grant 1RF1MH120679-01 to Davi Bock and G.S.X.E.J., with software development effort and administrative support provided by Tom Kazimiers (Kazmos GmbH) and Eric Perlman (Yikes LLC). We are also grateful to the Seung and Murthy labs for access to the flywire.ai reconstruction community. We thank Kei Ito, Masayoshi Ito and Shin-ya Takemura for the examination and discussion of neuron types and names in the antennal lobe and lateral horn, and Rachel Wilson for those in the antennal lobe. We also thank Romain Franconville for his contributions to our $\mathrm{R}$ package neuprintr. We thank Karen Menuz, Darya Task, Veit Grabe, Chris Potter and Silke Sachse for discussions on posterior antennal lobe glomeruli and receptor neuron identity. Finally, we thank Liqun Luo, Kei Ito, Rachel Wilson, Thomas Riemensperger and Andrew Lin for comments on the manuscript.

\section{Methods}

\section{Data and tool availability}

Hemibrain version 1.1 and 1.2 data is available via neuPrint (https://neuprint.janelia.org/) (Clements et alo, 2020; Scheffer et al., 2020). New FAFB tracing data presented in this study will be made available through the public CATMAID instance hosted by Virtual Fly Brain (https://fafb.catmaid. virtualflybrain.org/) upon publication. Previously published FAFB data is already available on the site.

Analyses were performed in R and in Python using open source packages. As part of this paper we have developed various new packages to fetch, process and analyse hemibrain data and integrated them with existing neuroanatomy libraries (Bates et al., 2020a). Table 1 gives an overview of the main software resources used. The packages used for specific analyses will be identified in each section of our methods.

Where appropriate, we have added short tutorials to the documentation of above packages demonstrating some of the analyses performed in this paper. We also provide example code snippets directly related to the analyses in this paper at https://github.com/flyconnectome/2020hemibrain 
Table 1. $R$ and Python packages used and developed in this study.

\begin{tabular}{|c|c|c|c|c|}
\hline & Language & Name & Github repository & Description \\
\hline \multirow{9}{*}{$\begin{array}{l}\text { by the } \\
\text { authors }\end{array}$} & $\mathrm{R}$ & neuprintr & natverse/neuprintr & Query data from neuPrint \\
\hline & $\mathrm{R}$ & hemibrainr & natverse/hemibrainr & $\begin{array}{l}\text { Analyse hemibrain data and } \\
\text { metadata }\end{array}$ \\
\hline & $\mathrm{R}$ & catmaid & natverse/rcatmaid & $\begin{array}{l}\text { Query CATMAID data (e.g. for } \\
\text { FAFB) }\end{array}$ \\
\hline & $\mathrm{R}$ & \multicolumn{2}{|c|}{ nat.jrcbrainsnatverse/nat.jrcbrains } & $\begin{array}{l}\text { Map between brain templates } \\
\text { (inc hemibrain \& FAFB) }\end{array}$ \\
\hline & $\mathrm{R}$ & nat.nblast & natverse/nat.nblast & Morphological comparison \\
\hline & Python & navis & schlegelp/navis & $\begin{array}{l}\text { Query and process neuron } \\
\text { data }\end{array}$ \\
\hline & Python & $\begin{array}{l}\text { navis- } \\
\text { flybrains }\end{array}$ & schlegelp/navis-flybrains & $\begin{array}{l}\text { Map between brain templates } \\
\text { (inc hemibrain \& FAFB) }\end{array}$ \\
\hline & Python & pymaid & schlegelp/pymaid & $\begin{array}{l}\text { Query CATMAID data (e.g. for } \\
\text { FAFB) }\end{array}$ \\
\hline & Python & fafbseg & flyconnectome/fafbseg-py & $\begin{array}{l}\text { Work with autosegmented } \\
\text { FAFB data (e.g. FlyWire) }\end{array}$ \\
\hline $\begin{array}{l}\text { third } \\
\text { party }\end{array}$ & Python & $\begin{array}{l}\text { neuprint- } \\
\text { python }\end{array}$ & $\begin{array}{l}\text { connectome- } \\
\text { neuprint/neuprint-python }\end{array}$ & $\begin{array}{l}\text { Query data from neuPrint, } \\
\text { developed by Stuart Berg } \\
\text { (Janelia Research Campus) }\end{array}$ \\
\hline
\end{tabular}

\section{Neuronal reconstructions in the hemibrain data set}

The hemibrain connectome (Scheffer et al., 2020) has been largely automatically reconstructed using flood-filling networks (Januszewski et al., 2018) from data acquired by focused ion-beam milling scanning EM (FIB-SEM) (Knott et al., 2008; Xu et al., 2017), followed by manual proofreading. Pre - (T-bars) and postsynapses were identified completely automatically. Significantly, the dense labelling allows estimating completion status as fraction of postsynapses successfully mapped to a neuron. For this first iteration of the hemibrain data set, the completion rate varies between $85 \%$ and $16 \%$ across neuropils. Notably, the lateral horn currently has one of the lowest completion rates with only $\sim 18 \%$ of postsynapses connected mapped to a neuron. We have therefore employed focused semi-manual review of identified neurons in the hemibrain for higher-fidelity connectivity comparison (no manual assessment of synapses). The data can be accessed via the neuPrint connectome analysis service (https://neuprint.janelia.org/) (Clements et al., 2020). We built additional software tools to pull, process and analyse these data for R (as part of the natverse ecosystem) (Bates et al., 2020a) and Python (see table above). Neurons can be read from neuPrint and processed (e.g. split into axon and dendrite) with the package hemibrainr using the function hemibrain_read_neurons.

\section{Neuronal reconstructions in the FAFB data set}

Unlike the hemibrain, the FAFB image volume comprises an entire female fly brain (Zheng et al., 2018). Two public segmentations of FAFB exist from Google (Li et al., 2019) and the Seung lab (https://flywire.ai/) (Dorkenwald et al., 2020). However, unlike for the hemibrain data set, these segmentations have not yet been proof-read by humans (at least not at scale). To date, most of the neuronal reconstruction in FAFB has been manual, using CATMAID (Saalfeld et al., 2009; Schneider-Mizell et al., 2016). We estimate that $\sim 7 \%$ of the brain's total neuronal cable, and $<1 \%$ 
of its connectivity, has been reconstructed in FAFB by a consortium of 27 laboratories worldwide using CATMAID. For data presented in this work, we have combined coarse morphologies extracted and proof-read from the FlyWire and Google segmentation with detailed manual reconstructions and synapse annotation. We have built software tools to pull, process and analyse these data from CATMAID and FlyWire in R (part of the natverse ecosystem) and Python.

\section{Processing of neuron skeletons and synapse data}

Raw skeleton and predicted synapse information from the hemibrain project may have a number of associated issues. Synapses, for example, are sometimes assigned to a neuron's soma or cell body fibre; these are incorrect automatic synapse detections. Autapses are often seen, but the majority of these cases are false-positives (the neuPrint web interface filters those by default). A single neuron may also have multiple skeletons associated with it that need to be connected. In addition, these skeletons are typically not rooted to their base - i.e. the soma if available or, in case of truncated neurons without a soma, the severed cell body fibre. A correctly rooted skeleton is important for some forms of analysis, including axon-dendrite splitting (Schneider-Mizell et al., 2016).

We wrote custom code to deal with these issues, as well as split neurons into their axon and dendrite. The correct root of a neuron was identified using an interactive pipeline and expert review (hemibrain_somas). We re-rooted all neurons in the data set (hemibrain_reroot), removed incorrect synapses at somata, along cell body fibres and along primary dendrites (hemibrain_remove_bad_synapses), healed split skeletons, employed a graph-theoretic algorithm to split neurons into axon and dendrites (hemibrain_flow_centrality) and implemented interactive pipelines for users to correct erroneous splits and soma placements. This has enabled us to build putative connectivity edge lists including neuron compartment information (hemibrain_extract_synapses). We have made our code and manipulated data available in our R package hemibrainr.

\section{Matching neurons between data sets}

Hemibrain neurons were matched to those from FAFB, as well as light level reconstructions (e.g. hemilineage models, see Wong et al. (2013); Lovick et al. (2013), stochastic labelling data (Dolan et $a l_{.}, 2019$ ) and images of neuron clones (Yu et al., 2013; Ito et al., 2013) by bridging these data into the same brain space (Bogovic et al., 2020; Bates et al., 2020a) and then using NBLAST (Costa et al., 2016) to calculate neuron-neuron morphology similarity scores.

Neurons were bridged using the R nat.jrcbrains package (https://github.com/natverse/ nat.jrcbrains) and nat.templatebrains::xform_brain function or the Python package navis (navis.xform_brain) in combination with navis-flybrains (https://github.com/schlegelp/navis-flybrains), both of which wrap light-EM bridging registrations reported in Bogovic et al. (2020). Prior to NBLAST (using nat.nblast or navis), EM skeletons were scaled to units of microns, arbour was resampled to $1 \mu \mathrm{m}$ step size and then converted to vector cloud dotprops format with $\mathrm{k}=5$ neighbours. To ensure that skeletons from the two EM data sets could be fairly compared, we performed certain post-processing steps such as pruning away terminal twigs of less than $2-5 \mu \mathrm{m}$ (nat: : prune_twigs/navis.prune_twigs) or restricting the arbour for all neurons to the hemibrain volume (hemibrainr: : hemibrain_cut) (even if tracing existed outside of this volume for FAFB neurons).

For TOON matching, human experts then visually compared potential matches (with function hemibrain_matching) and qualitatively assessed them as 'good', a near-exact match between the two data sets; 'medium', match definitely represents neurons of the same cell type; and 'poor', neurons are probably the same cell type but under-tracing, registration issues or biological variability made the expert uncertain. We have made our matching pipeline code and matches available in our $\mathrm{R}$ package hemibrainr. Matches are available in the package hemibrainr as hemibrain_matches. 
855

856

857

858

859

860

861

862

\section{Neurotransmitter assignment}

We know the transmitter expression of a few hundred olfactory system neurons based mainly on immunohistochemistry results from the literature (Tanaka et al., 2012b; Wilson and Laurent, 2005; Liang et al., 2013; Lai et al., 2008; Dolan et al., 2019; Aso et al., 2014a; Okada et al., 2009; Tanaka et al., 2012a). To guess at the transmitter expression of related neurons, we hypothesised that if brain neurons share a hemilineage they will share their fast-acting transmitter expression, as has been seen in the adult ventral nerve cord (Lacin et al., 2019). If neuron 1 belongs to the same hemilineage as neuron 2, for which there is data to suggest its neurotransmitter expression, neuron 1 is assumed to express the same neurotransmitter.

\section{Antennal lobe glomeruli}

The antennal lobe is composed of 58 neuropils called glomeruli. Each glomerulus is a region where a specific type of olfactory or thermo/hygrosensory receptor neurons (ALRNs) synapses onto local and projection neurons, ALLNs and ALPNs, respectively. There are 7 identified thermo/hygrosensory glomeruli: VP1d, VP1l, VP1m (Marin et al., 2020), VP2, VP3 (Stocker et al., 1990), VP4 (Silbering et al., 2011; Frank et al., 2017; Knecht et al., 2017) and 51 olfactory glomeruli (Bates et al., 2020a).

\section{Truncated glomeruli}

Based on a qualitative assessment, a number of glomeruli (DA4I, DA4m, DM5, VA2, VC5, VM1, VM2, VM3) are substantially (>25\%) and 11 (D, DA2, DA3, VA1d, VA6, VA7m, VM6, VM4, VM5d, VM5v, VM7d) are partially $(<25 \%)$ truncated in the hemibrain. The truncation is due to the proximity of these glomeruli to the 'hot-knife' sections and to the boundary line in the imaging sample (medial and anterior antennal lobe regions).

\section{Renaming posterior AL glomeruli}

Our glomerular identification in Bates et al. (2020b) was principally based on previously reported projection neurons (ALPNs) associated with a single glomerulus (i.e. "uniglomerular PNs"). Using PNs provides more points of reference (position of dendrites, lineage, axonal projections) than the relative positions of sensory receptor neuron (ALRN) axon terminals. While this approach works for most glomeruli, some of the posterior glomeruli have had conflicting reports in the literature:

- VC3 has been treated as a single glomerulus (e.g. Yu et al. (2010)) as well as two separate glomeruli, VC3m and VC3I (e.g. Tanaka et al. (2012a); Laissue et al. (1999))

- the VM6 PN has been referred to as VC5 (Tanaka et al., 2012a) and VM6+VP1 (Yu et al., 2010)

In collaboration with Karen Menuz (University of Connecticut), Darya Task and Chris Potter (John Hopkins University), Veit Grabe and Silke Sachse (Max Planck Institute for Chemical Ecology), we now consolidate these accounts with extant literature on sensory receptors (see also below table). As a result of this, three glomeruli were renamed compared with hemibrain v1.1/v1.2 and Bates et al. (2020b):

- VC3I $\rightarrow$ VC3

- VC3m $\rightarrow$ VC5

$\cdot$ VC5 $\rightarrow$ VM6

ALRNs of the VM6 glomerulus further split into three distinct subpopulations - VM6V, VM6m and VM6I - with different receptors and origins ((Task et al., 2020)) (Figure 3-Figure Supplement 2B). This is likely part of the reason for confusion in the past. Because these subpopulations appear to be indiscernible from the perspective of the downstream network (Figure 3-Figure Supplement $2 \mathrm{~B}, \mathrm{C}$ ), we decided to refer to the PNs that cover the combination of $\mathrm{VM} 6 \mathrm{~V}$, VM6m and VM6I as "VM6" uPNs. Following this reasoning, we still refer to the antennal lobe as containing 58 glomeruli. Please see Table 2 for a summary and supporting references. 
Table 2. Names of posterior glomeruli across datasets and publications, and supporting reference for names used in this study.

\begin{tabular}{|c|c|c|c|c|c|c|}
\hline glomerulus & $\begin{array}{c}\text { hemibrain } \\
\text { v1.1+1.2 }\end{array}$ & $\begin{array}{l}\text { Bates } \\
\text { et al. } \\
(2020)\end{array}$ & $\begin{array}{c}\text { Tanaka } \\
\text { et al. } \\
(2012\end{array}$ & $\begin{array}{c}\text { Yu et al. } \\
\text { (2010) }\end{array}$ & receptor & $\begin{array}{l}\text { supporting } \\
\text { references }\end{array}$ \\
\hline VC5 & VC3m & VC3m & VC3m & - & Ir41a & $\begin{array}{l}\text { Silbering et al. } \\
\text { (2011); Task et al. } \\
\text { (2020); Hussain } \\
\text { et al. (2016); } \\
\text { Min et al. (2013); } \\
\text { Chai et al. (2019) }\end{array}$ \\
\hline VC3 & VC3I & VC3I & VC3I & VC3 & Or35a & $\begin{array}{l}\text { Couto et al. } \\
\text { (2005); Grabe } \\
\text { et al. (2016); } \\
\text { Silbering et al. } \\
\text { (2011); Task et al. } \\
\text { (2020); Min et al. } \\
(2013)\end{array}$ \\
\hline VM6 $(v+m+l)$ & VC5 & VC5 & VC5 & VM6+VP1 & Rh50/Amt & $\begin{array}{l}\text { Endo et al. } \\
\text { (2007); Li et al. } \\
\text { (2016); Chai et al. } \\
\text { (2019); Vulpe } \\
\text { et al. (2021); } \\
\text { Task et al. (2020) }\end{array}$ \\
\hline
\end{tabular}

These corrections affect names ("instances") and types of ALRNs and ALPNs. Changes will be merged into the hemibrain with the release of version 1.3. All neurons can still be unambiguously identified and tracked across versions of the dataset via their body IDs.

AL glomeruli meshes

The boundary between glomeruli can be defined either using presynapses of ALRNs or the corresponding postsynapses of uniglomerular ALPNs (UPNs). Hence we generated both ALRN - and ALPN-based glomeruli meshes. These are available in the package hemibrainr as hemibrain_al.surf and in the supplemental data.

In brief, we used the location of synapses (either dendritic postsynapses of identified uPNs or axonal presynapses of ALRNs) to produce a Gaussian kernel density estimate (KDE) for each glomerulus. We then divided the entire AL into isotropic 480nm voxels and used the KDEs' point density functions (pdf) to assign each voxel to its most likely glomerulus. Voxels with a belowthreshold probability to belong to any glomerulus (e.g. tracts) were discarded. The voxel data was postprocessed (binary erosion, fill holes) before being converted to meshes using a marching cubes algorithm. All above steps were performed in Python using scipy (https://www.scipy.org) and scikit-learn (https://scikit-learn.org). Sample code can be found at https://github.com/flyconnectome/ 2020hemibrain_examples. Finally, the meshes were inspected and manually fixed if required using Blender3d (https://www.blender.org). For the ALPN-based glomeruli meshes, we used the location of dendritic postsynapses of all the uniglomerular projection neurons (UPNs) - except for glomeruli VP3, VP5, VP1d, VP1I which do not have clear-cut uPNs and where we used the presynaptic locations of corresponding ALRNs. For the ALRN-based meshes we used locations of ALRN presynapses. Here, VM2 was excluded because of too few RNs identified for this glomerulus. Also note that for the ALRN-based meshes we used the VM6 ORN subtypes to generate separate meshes 
for VM6v, VM6m and VM6I.

\section{Cell type annotation}

Annotations are available via neuPrint and as part of our $\mathrm{R}$ package hemibrainr. These are available in the package hemibrainr with the function hemibrain_get_meta.

\section{Antennal lobe receptor neurons}

Antennal lobe receptor neurons (ALRNs, 2643) were identified by morphology and by connectivity to projection neurons. Types were named by the glomerulus they innervate. Soma side was assigned to each ALRN from non-truncated glomeruli whenever possible, based on visual inspection of the path of the neurite towards the nerve entry point.

The number of ALRNs in the 39 whole glomeruli is 1680. For 8 types (DC3, VA1V, VA3, VA4, VA5, VA7I, VC2, VC4), although the glomeruli are whole, the majority of ALRNs are fragmented, preventing the assignment of a soma side. For VM6 ALRNs, the glomeruli truncation prevented us from assigning every VM6 ALRN to one of the 3 populations (12 unassigned). For that reason, in certain instances, we still refer to VM6 ALRNs as one group.

Particularly in truncated glomeruli and glomeruli with fragmented ALRNs, there are many smaller, and fragmented bodies for which it is not possible to say if they represent a unique ALRN, or if they will merge to another body. Although we have tried to identify these fragments we cannot be sure that the total number of ALRN bodies is an accurate representation of the number of ALRNs.

In addition to the 2644 ALRNs that we were able to classify, there were 10 that presented issues. Two could be identified as ALRNs but their glomerular arborisation was missing, therefore a type could not be assigned (ids 2197880387, 1852093746, not listed in Supplementary File). Three typed ORNs were excluded because they were pending fixes that altered their connectivity (ids 1951059936, 2071974816, 5812995304). We also found 5 outlier ORNs with axon terminals not confined to one glomerulus (either 2 glomeruli in one hemisphere, different glomeruli between hemispheres or innervating the antennal lobe hub (ids 1760080402, 1855835989, 2229278366, 2041285497, 5813071357).

To assess potential subdivisions of ALRN populations within each glomerulus (Figure 3-Figure Supplement 2), we used a modified version of the synapse-based morphological clustering in Schlegel et al. (2016) coined syNBLAST (implemented in our Python library navis).

\section{Antennal lobe local neurons}

Candidate neurons (4973) were first identified as any neuron that had at least $5 \%$ of its pre - or post-synapses in the AL. From these we excluded the already typed ALPNs (338) and ALRNs (2653), resulting in a candidate list (307) of antennal lobe local neurons (ALLNs). Among these only 197 could be typed in accordance with their lineage, morphology and connectivity. The remaining 110 ALLNs are too fragmented to classify and were not used further. Only the ALLNs from the right hemisphere (196) were included in the analysis.

Lineages were identified on the basis of soma and cell body fibre location, partially shared with ALPNs. Next, major groups were assigned in accordance with the previously described neurite morphologies (Chou et al., 2010). Due to truncated glomeruli in the data set, we decided to not distinguish between ALLNs innervating all but a few glomeruli vs most glomeruli; thus both groups are classified as broad ALLNs. The 74 cell types were assigned based on the major morphology class, presence/absence of a bilateral projection, glomerular innervation patterns and neurite density. The ALLN types were named by concatenating lineage, ID number/capital letter combination and a small letter, in case of strong connectivity differences. The first 6 ID numbers match the previously identified ALLN types in Tanaka et al. 2012, and the following are newly identified types, in decreasing order of arbour size. 
Antennal lobe projection neurons

Uniglomerular ALPNs (UPNs) were identified by morphology and classified according to our recent complete inventory from the FAFB data set by matching neurons with the help of NBLAST (Bates et al., 2020b). Multiglomerular ALPNs (mPNs) not been comprehensively typed in past studies. Therefore, mPNs types for hemibrain v1.1/v1.2 were determined in coordination with Kei Ito, Masayoshi Ito and Shin-ya Takemura using a combination of morphological and connectivity clustering. These v1.1/v1.2 mPN types were deliberately very fine-grained to facilitate potential changes (e.g. merges) future releases. See also the paragraph on ALPN analyses below.

\section{Non-MB olfactory third-order neurons}

Non-MB olfactory third-order olfactory neurons (TOONs) were defined as neurons downstream of ALPN axons outside of the MB calyx. They must receive $1 \%$ of their synaptic input (or else 10 connections) from an olfactory ALPN, or otherwise $10 \%$ of their input (or else 100 connections) from any combination of olfactory ALPNs. This search yields 2383 identifiable, and mainly complete, neuron morphologies. TOONs comprise a range of neuron classes, including a small number of second and third-order neurons of the gustatory, mechanosensory and visual systems, as well as dopaminergic neurons of the mushroom body, descending neurons to the ventral nervous system and, most prominently, neurons of the lateral horn.

\section{Lateral horn neurons}

Lateral horn neurons (LHNs) were defined as a subset of TOONs that have at least 10 pre - or postsynapses in the LH volume (as defined in the hemibrain). We named these cells by extending the LHN naming scheme from Frechter et al. (2019), except for cell types with more prominent names already in use in the literature. Neurons were first divided into their hemilineages, indicated by the path of their cell body fibres, e.g. DPLm2 (Lovick et al., 2013). Hemilineage matches were made to both FAFB and light-level data in order to verify their composition. To simplify the naming of neurons, hemilineages and primary neurons (those cells born in the embryo, which do not fasciculate strongly with secondary hemilineages in the adult brain) were grouped into similar-looking groups, e.g. PV5 (posterior-ventral to the LH, 5). Next, neurons within each hemilineage were grouped into coarse morphological sets, termed 'anatomy groups', e.g. PV5a. Within each anatomy group, LHNs were broken into morphological cell types using NBLAST, followed by manual curation, e.g. PV5a1. Partial reconstructions in FAFB, concatenated using automatically reconstructed neuron fragments

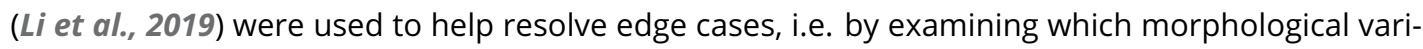
ations appeared consistent between data sets. Neurons were further subdivided into connectivity types (i.e. 'cell type_letter') using CBLAST (Scheffer et al., 2020), e.g. LHPV5a1_a. With so many new types being added, our expansion of the Frechter et al. (2019) LH naming system incurred some changes. We have tried to keep names used in main sequence figures in our previous publications (Dolan et al., 2019; Frechter et al., 2019; Bates et al., 2020b) but some have changed as, for example, the hemibrain data has revealed that neurons originate from a different hemilineage or neurons we had once considered to be of the same cell type have different connectivity profiles. Code for these analyses can be found in our R data package, Ihns and hemibrainr.

\section{Descending neurons}

The hemibrain v1.1/v1.2 data set includes cell type information for 109 descending neurons (DNs) (Namiki et al., 2018), 88 with somata on the right hand side of the brain. Given that the hemibrain volume does not include the neck connective, ambiguous or previously unknown DNs are difficult to identify. We sought to identify as many DNs as possible without explicitly defining the cell types (many of which are not previously reported in the literature). We used several data sources to help identify DNs including manual and automated tracing in FAFB (Zheng et al., 2018; Li et al., 2019) and the neuronbridge search tool (https://neuronbridge.janelia.org/, https://github.com/JaneliaSciComp/ neuronbridge, (Meissner et al., 2020; Otsuna et al., 2018), also see our R package neuronbridger). 
The single most comprehensive source of information is the recent FlyWire segmentation of the FAFB volume (https://flywire.ai/) (Dorkenwald et alo, 2020) where we reconstructed neurons that descend from the brain through the neck connective. These FAFB DNs were cross-matched against all hemibrain neurons using NBLAST and subsequent manual curation. This enabled us to identify an additional 236 hemibrain hemibrain neurons as DNs (see Supplementary Files). A detailed cell typing of these DNs based on combining both data sets will be presented in a future manuscript.

\section{Graph traversal model}

To sort hemibrain neurons into layers with respect to the olfactory system we employ a simple probabilistic graph traversal model. The model starts with a given pool of neurons - receptor neurons (ALRNs) in our case - as seeds. It then pulls in neurons directly downstream of those neurons already in the pool. This process is repeated until all neurons in the graph have been "traversed" and we keep track of at which step each neuron was visited. Here, the probability of a not-yet-traversed neuron to be added to the pool depends on the fraction of the inputs it receives from neurons already in the pool. We use a linear function to determine the probability $P_{i j}$ of a traversal from neuron $i$ to $j$ :

$$
P_{i j}= \begin{cases}\frac{w_{i j}}{\left(\sum_{k} w_{k j}\right) * 0.3} & \text { if } P_{i j} \leq 1 \\ 1 & \text { if } P_{i j}>1,\end{cases}
$$

where $w_{i j}$ is the number of synaptic connections from $i$ to $j$. In simple terms: if the connection from neuron $i$ makes up 30\% or more of neuron $j^{\prime}$ s inputs, there is a 100\% chance of it being traversed. Each connection from a neuron already in the pool to a neuron outside the pool has an independent chance to be traversed. The threshold of $30 \%$ was determined empirically such that known neuron classes like ALLNs and ALPNs are assigned to the intuitively "correct" layer.

The graph traversal was repeated 10,000 times for the global models (Figure 2 and Figure 2Figure Supplement 1) and 5,000 per type for the by-RN-type analysis (Figure 14). Layers were then produced from the mean across all runs. The code for the traversal model is part of navis (https: //github.com/schlegelp/navis).

To generate the graph, we used all hemibrain v1.2 neurons with either a type annotation or status label "Traced" or "Roughly traced". We then took the edges between those neurons and removed (a) single-synapse connections to reduce noise and (b) connections between Kenyon cells which are considered false positives (Li et al., 2020). This produced a graph encompassing 12.6M chemical synapses across $1.7 \mathrm{M}$ edges between $24.6 \mathrm{k}$ neurons. Outputs of the model as used in this paper are available in the package hemibrainr as hemibrain_olfactory_layers.

\section{Class-compartment separation score}

This score is inspired by the synapse segregation index used in (Schneider-Mizell et al., 2016). ALPN innervation of a dendrite is first normalised by the total amount of innervation by ALPNs ( $d$.pn) and $\operatorname{MBONs}($ d.mbon):

$$
\text { d.total }=\text { d.mbon }+d \cdot p n
$$

$$
D=d . p n / d . t o t a l
$$

A dendrite segregation index is then calculated as:

$$
d . s i=-\left(D * \log _{10}(D)-(1-D) * \log _{10}(1-D)\right)
$$

Where $D$ is the proportion of dendritic innervation by ALPNs, divided by the total dendritic innervation by MBONs and ALPNs. The axon segregation index (a.si) is calculated for the axon of the same neuron. Then the entropy is taken as: 


$$
\begin{gathered}
e=(1 /(\text { d.total }+ \text { a.total }) *((\text { a.si } * \text { a.total })+(\text { d.si } * \text { d.total })) \\
P N=(\text { d.pn }+ \text { a.pn }) /(\text { d.total }+ \text { a.total }) \\
c=-\left(P N * \log _{10}(P N)+(1-P N)\right) \\
\text { segregation.score }=1-(e / c)
\end{gathered}
$$

\section{Antennal lobe receptor neuron analyses}

ALRN analysis included only those ALRNs for which a glomerular type has been assigned and it excluded glomeruli that are truncated (see 'Antennal lobe glomeruli'). Additionally, any analysis that relied on soma side excluded the 8 types that have whole glomeruli but have truncated ALRNs (DC3, VA1v, VA3, VA4, VA5, VA7I, VC2, VC4). Only bilateral ORNs were used for laterality comparisons, as only 1 of 7 TRN/HRN types is bilateral.

In connectivity plots, the category 'other' includes any neuron that has been identified, but is not an ALRN, ALPN or ALLN. 'Unknown' refers to un-annotated bodies; this might include potential ALRN fragments that cannot be identified.

ALRN presynaptic density was calculated using skeletons and presynapses subsetted to the relevant ALRN-based glomerulus mesh.

\section{Antennal lobe projection neuron analyses}

\section{Across-dataset morphological clustering}

For clustering ALPNs across data sets (hemibrain vs FAFB right vs FAFB left) we first transformed their skeletons from their respective template brains to the JRC2018F space. FAFB left ALPNs were additionally mirrored to the right (Bogovic et al., 2020; Bates et al., 2020a). We then used NBLAST to produce morphological similarity scores between ALPNs of the same (hemi-)lineage (Costa et al., 2016). For NBLASTs between hemibrain and FAFB ALPNs, the FAFB ALPNs were first pruned to the hemibrain volume such that they were similarly truncated. The pairwise NBLAST scores were generated from the minimum between the forward (query $\rightarrow$ target) and reverse (query $\leftarrow$ target) scores.

Next, we used the NBLAST scores to - for each ALPN - find the best matches among the ALPNs in the other two data sets. Conceptually, unique ALPNs should exhibit a clear 1:1:1 matching where the best across-dataset match is always reciprocal. For ALPN types with multiple representatives we expect that individuals can not be tracked across dataset because matches are not necessarily reciprocal. We used a graph representation of this network of top matches to produce clusters (Figure 6-Figure Supplement 1B). These initial clusters still contained incorrect merges due to a small number of "pathological" ALPNs (e.g. from developmental aberrations) which introduce incorrect edges to the graph. To compensate for such cases, we used all pairwise scores (not just the top NBLAST scores) to refine the clusters by finding the minimal cut(s) required to break clusters such that the worst within-cluster score was $>=0.4$ (Figure 6-Figure Supplement 1C). This value was determined empirically using the known uPN types as landmarks. Without additional manual intervention, this approach correctly reproduced all "canonical" (i.e. repeatedly described across multiple studies) UPN types. We note though that in some cases this unsupervised clustering still requires manual curation. We point out some exemplary cases in Figure 6-Figure Supplement 1FJ. For example, M_adPNm4's exhibit features of uniglomerular VC3 adPNs and as a result are incorrectly co-clustered with them. Likewise, a single VC5 IVPN invades the VM4 glomerulus and is therefore co-clustered with the already rather similar looking VM4 IVPNs. In such cases, connectivity information could potentially be used to inform the refinement of the initial clusters. 
1100

1101

1102

1103

1104

1105

1106

1107

1108

1109

1110

1111

1112

1113

1114

1115

1116

1117

1118

1119

1120

1121

1122

1123

1124

1125

1126

1127

1128

1129

1130

1131

1132

1133

1134

1135

1136

1137

1138

1139

1140

1141

1142

1143

1144

1145

1146

\section{Connectivity}

Analyses of ALPN connectivity excluded glomeruli that are truncated (see 'Antennal lobe glomeruli'). Additionally, any analysis that relied on ALRN soma side (i.e. ipsilateral ALRNs versus contralateral) excluded the 8 glomeruli that are whole but have truncated ALRNs (DC3, VA1V, VA3, VA4, VA5, VA7I, $V C 2$, VC4). In connectivity plots, the category 'other' includes any neuron that has been identified, but is not an ALRN, ALPN or ALLN. 'Unknown' refers to un-annotated bodies; this might include potential RN fragments that cannot be identified.

\section{Antennal lobe local neuron analyses}

The main theme of the ALLN analysis is to quantify the differences across ALLN types (based on morphology) in innervation (synapses across glomeruli, co-innervation, intra-glomerular morphology) and connectivity motifs. For all of the ALLN analysis, glomerular meshes based on the ALPNbased glomeruli were used.

\section{Synaptic distribution across glomeruli}

The main goal of this analysis was to understand how synapses are distributed across the glomeruli, for the ALLN types. First, for each morphological type, we constructed a matrix with columns representing neurons and rows representing glomeruli. Each element in this matrix has the number of synapses of the specific neuron in the corresponding glomerulus. Synapses per neuron were fetched using the neuprint-python package (Python, https://github.com/connectome-neuprint/ neuprint-python). Second, for each neuron, glomerular identities were collapsed and sorted by descending order. Third, each column (neuron) was normalised from a range of 0 to 1 using the minmax scaler from the scikit-learn (Python, https://scikit-learn.org/) package. Fourth, the cumulative sum per column was computed. The resulting matrix is composed of each column (neuron) where synaptic score is ordered in a cumulative way.

\section{Glomerular co-innervation}

The main goal of this analysis was to identify pairs of glomeruli that are strongly co-innervated by different ALLN types. For defining co-innervation, the number of synapses in the specific glomeruli from the specific neuron would be used. First, for each morphological type, we constructed a matrix where columns represented neurons and rows represented glomeruli. Each element in this matrix reflected the number of synapses of that neuron in that specific glomerulus. Synapses per neuron were fetched using the neuprint-python package. Second, the possible combinations of pairs of glomeruli (that are un-cut) was computed: $39 \mathrm{C}_{2}$ or 741 total pairs. Third, for each combination pair the synapses that are co-occurring within a neuron were calculated, resulting in a matrix of dimensions combination pairs (741) by number of neurons of specific ALLN type. Fourth, co-occurring synapses per pair were summed, resulting in a vector of length combinations. This represented the ground truth of co-occurring synapses. Fifth, after computing the matrix from step 3 , we shuffled every row independently (i.e. choosing a neuron and shuffling across the pairs of glomeruli). Sixth, we then performed step 4 with this shuffled matrix and repeated steps 5 and 6 for $20 \mathrm{k}$ times. This output represented the shuffled synapses. Seventh, for each pair of glomeruli, we computed the proportion of shuffled synapses (within a specific pair of glomeruli) that are higher than the ground truth; this conveys the likelihood of the ground truth being non-random and hence it is the uncorrected p-value. Lastly, we corrected the p-value for multiple comparisons using the package statsmodels (Python, https://www.statsmodels.org/), using the holm-sidak procedure with a family wise error rate of 0.05 . The pairs with significant $p$-values following the correction represent the pairs of glomeruli that are strongly co-innervated by the specific ALLN type.

\section{Connectivity}

The main goal of this analysis was to identify how different ALLN types are connected to olfactory ALRNs, uPNs, mPNs, and thermo/hygrohygrosensory ALPNs. The input and output synapses be- 
tween ALLNs and other categories were fetched using the neuprint-python package. ALLNs were categorised into a combination of morphological type (sparse, etc) and lineage type ( $v$, etc).

\section{Intra-glomerular morphology}

The main goal of this analysis was to identify how intra-glomerular innervation patterns vary across different ALLN types. First, taking each whole glomerulus in turn, we pruned the arbors for each ALLN within that glomerulus using the navis package (Python, https://github.com/schlegelp/navis/). From the pruned ALLNs we excluded any with less than 80 micrometres of cable length. Second, we calculated the distance between all pairs of ALLNs within that specific glomerulus. This was done as follows: first, for each ALLN pair, for each node we took the 5 nearest nodes in the opposite ALLN using the KDTree from the scipy package (Python, https://www.scipy.org/) and further computed the mean distance. Second, the same procedure was then repeated for all nodes on both sets of ALLNs, producing mean distances per node per ALLN. Lastly, we collapsed the ids of the neurons and computed the mean of the top 10\% (largest) of the mean distances. This was considered to be the mean intraglomerular distance between the ALLNs for that specific glomerulus.

\section{Input-Output segregation}

The main goals of this analysis were 1) to identify how different ALLN morphological classes vary in the amount of synaptic input and output across different glomeruli and 2) to compare the same with UPNs and ALRNs. First, for each type, we constructed a presynaptic matrix where columns represented neurons and rows represented glomeruli. Each element in this matrix reflected the number of presynaptic connectors of that neuron in that specific glomerulus. Connectors per neuron were fetched using the neuprint-python package. Similarly, we constructed a postsynaptic matrix, where each element reflected the number of postsynapses of that neuron in that specific glomerulus. Second, we performed postprocessing on both the presynaptic and postsynaptic matrix. For each neuron, we ranked glomeruli in descending order by synapse number and then removed those glomeruli accounting for the bottom $5 \%$ of the synapses. Third, we computed the difference (Input-Output segregation) by subtracting presynaptic connectors from the postsynapses per neuron. Here we ignored glomeruli where both presynaptic connectors and postsynapses are zero. Fourth, we collapsed the glomerular identities and sorted all neurons by the difference (InputOutput segregation). Finally, we computed the mean across the neurons. We gave positive ranks to values above 0 (more input) and negative ranks to values below 0 (more output).

Clustering of ALLNs by the ratio of their axonal output or dendritic input per glomerulus The main goal of this analysis (Figure 4-Figure Supplement 1G) was to identify how different ALLN types are polarised across different glomeruli (axon-dendrite split developed using the algorithm from Schneider-Mizell et al. (2016)). First, we selected only those ALLNs (76) that have a axodendritic segregation index of $>0.1$, i.e. they are polarised. Second, for each ALLN we computed the axon and dendritic compartment using the flow-centrality algorithm developed in SchneiderMizell et al. (2016). Third, for each glomerulus and for each ALLN we computed the fraction of dendritic inputs (input synapses located in the dendritic compartment inside the specific glomerulus) to the total dendritic inputs (input synapses located in the dendritic compartment across all glomeruli) and fraction of axonic outputs (output synapses located in the axonic compartment inside the specific glomerulus) to the total axonic outputs (output synapses located in the axonic compartment across all glomeruli). Fourth, we computed a score defined by the fraction of axonic output - the fraction of dendritic input. The higher the score, the greater the ALLN's bias for axonically outputting in a glomerulus, over receiving dendritic input. Fifth, we computed the mean of these scores for different ALLN types across the different glomeruli. Finally, we applied the clustering algorithm (using hierarchical clustering based on Ward's distance using functions from base R) to these scores. 
1194

1195

\section{Supplemental data}

We have made our code, with examples, and detailed data available in our R package hemibrainr. Here we provide core data. Please see Table 3 for a description of the meta data contained in the supplemental files.

\section{Supplemental file 1}

Layers assigned by the probabilistic graph traversal model. bodyId refers to neurons' unique ID in neuPrint. layer_mean contains the mean layer after 10,000 iterations of the main model (Figure 2). layer_olf_mean and layer_th_mean contain the mean layers from running the traversal model with ORNs and THN/HRNs, respectively (Figure 2-Figure Supplement 2).

S1_hemibrain_neuron_layers.csv

\section{Supplemental file 2}

Sensory meta-information related to each glomerulus. Columns: glomerulus (canonical name for one of the 51 olfactory +7 thermo/hygrosensory antennal lobe glomeruli), laterality (whether the glomerulus receives bilateral or only unilateral innervation from ALRNs), expected_cit (a citation that describes the expected number of RNs in this glomerulus), expected_RN_female_1h (number of expected RNs in one hemisphere), expected_RN_female_SD (standard deviation in the expected number of RNs), missing (qualitative assessment of glomeruli truncation), RN_frag (if the RNs in that glomerulus are fragmented), receptor (the OR or IR expressed by cognate ALRNs, (Bates et al., 2020b; Task et al., 2020)), odour_scenes (the general 'odour scene(s)' which this glomerulus may help signal, (Mansourian and Stensmyr, 2015; Bates et al., 2020b)), key_ligand (the ligand that excites the cognate ALRN or receptor the most, based on pooled data from multiple studies, Münch and Galizia (2016)), valence (the presumed valence of this odour channel, Badel et al. (2016)). Exists as hemibrain_glomeruli_summary in our R package hemibrainr.

S2_hemibrain_olfactory_information.csv

\section{Supplemental file 3}

File listing all identified antennal lobe receptor neurons (ALRNs) in the hemibrain, including information shown in neuPrint. See above for column explanations. Exists as rn. info in our R package hemibrainr.

S3_hemibrain_ALRN_meta.csv

\section{Supplemental file 4}

All the hemibrain neurons we have classed as antennal lobe local neurons (ALLNs). See above for column explanations. Exists as alln. info in our R package hemibrainr.

S4_hemibrain_ALLN_meta.csv

\section{Supplemental file 5}

All the hemibrain neurons we have classed as antennal lobe projection neurons (ALPNs). See above for column explanations. In addition, across_dataset_cluster refers to the clustering with left and right FAFB PNs; is_canonical indicates whether that ALPN is one of the well studied "canonical" uPNs. Exists as pn. info in our R package hemibrainr.

S5_hemibrain_ALPN_meta.csv 
1233

1234

1235

1236

1237

1238

1239

\section{Supplemental file 6}

All the hemibrain neurons we have classed as third-order olfactory neurons (TOONs) including lateral horn neurons (LHNs), as well as wedge projection neurons (WEDPNs), lateral horn centrifugal neurons (LHCENT) and other projection neuron classes (Figure 1). See above for column explanations. Exists as ton.info in our R package hemibrainr.

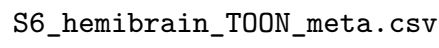

\section{Supplemental file 7}

All the hemibrain neurons we have classed as neurons that descend to the ventral nervous system (DNs). See above for column explanations. Exists as dn. info in our $\mathrm{R}$ package hemibrainr. S8_hemibrain_DN_meta.csv

\section{Supplemental file 8}

The root point in hemibrain voxel space, for each hemibrain neuron. This is either the location of the soma, or the tip of a severed cell body fibre tract, where possible. Exists as hemibrain_somas in our R package hemibrainr.

S8_hemibrain_root_points.csv

\section{Supplemental file 9}

The start points for different neuron compartments. Nodes downstream of this position in the 3D structure of the neuron indicated with bodyid, belong to the compartment type designated by Label. A product of running flow_centrality on hemibrain neurons, exists as hemibrain_splitpoints in our R package hemibrainr.

S9_hemibrain_compartment_startpoints.csv

\section{Supplemental file 10}

3D triangle mesh for the hemibrain surface as a .obj file. This mesh was generated by first merging individual ROI meshes from neuPrint and then filling the gaps in between in a semi-manual process. It also exists as hemibrain. surf in our R package hemibrainr.

$$
\text { S10_hemibrain_raw.obj }
$$

\section{Supplemental file 11}

3D meshes of 51 olfactory +7 thermo/hygrosensory antennal lobe glomeruli for the hemibrain volume, generated from ALRN presynapses. These meshes follow the subdivision of VM6 and hence contain 60 meshes in total.

Note that hemibrain coordinate system has the anterior-posterior axis aligned with the $Y$ axis (rather than the $\mathrm{Z}$ axis, which is more commonly observed).

S11_hemibrain_AL_glomeruli_meshes_RN-based.zip

\section{Supplemental file 12}

3D meshes of 51 olfactory +7 thermo/hygrosensory antennal lobe glomeruli for the hemibrain volume, generated from ALPN postsynapses.

Note that hemibrain coordinate system has the anterior-posterior axis aligned with the $Y$ axis (rather than the $Z$ axis, which is more commonly observed).

These meshes are also available as hemibrain_al .surf in our R package hemibrainr. 
bioRxiv preprint doi: https://doi.org/10.1101/2020.12.15.401257; this version posted May 14,2021 . The copyright holder for this preprint (which was not certified by peer review) is the author/funder, who has granted bioRxiv a license to display the preprint in perpetuity. It is made available under aCC-BY 4.0 International license.

1272

S12_hemibrain_AL_glomeruli_meshes_PN-based.zip 
bioRxiv preprint doi: https://doi.org/10.1101/2020.12.15.401257; this version posted May 14, 2021. The copyright holder for this preprint (which was not certified by peer review) is the author/funder, who has granted bioRxiv a license to display the preprint in perpetuity. It is made available under aCC-BY 4.0 International license.

1273

1274

1275

1276

1277

1278

1279

1280

1281

1282

1283

1284

1285

1286

1287

1288

1289

1290

1291

1292

1293

1294

1295

1296

1297

1298

1299

1300

1301

1302

1303

1304

1305

1306

1307

1308

1309

1310

1311

1312

1313

1314

1315

1316

1317

1318

1319

1320

1321

\section{References}

Abbott LF, Bock DD, Callaway EM, Denk W, Dulac C, Fairhall AL, Fiete I, Harris KM, Helmstaedter M, Jain V, Kasthuri N, LeCun Y, Lichtman JW, Littlewood PB, Luo L, Maunsell JHR, Reid RC, Rosen BR, Rubin GM, Sejnowski TJ, et al. The Mind of a Mouse. Cell. 2020 9; 182(6):1372-1376. doi: 10.1016/j.cell.2020.08.010.

Agarwal G, Isacoff E. Specializations of a pheromonal glomerulus in the Drosophila olfactory system. J Neurophysiol. 2011 4; 105:1711-21. doi: 10.1152/jn.00591.2010.

Amin H, Lin AC. Neuronal mechanisms underlying innate and learned olfactory processing in Drosophila. Curr Opin Insect Sci. 2019 6; 36:9-17. doi: 10.1016/j.cois.2019.06.003.

Aso Y, Hattori D, Yu Y, M JR, lyer NA, Ngo TTB, Heather D, Abbott LF, Axel R, Tanimoto H, Rubin GM. The neuronal architecture of the mushroom body provides a logic for associative learning. eLife. 2014 12; 3:e04577. doi: 10.7554/eLife.04577.

Aso Y, Sitaraman D, Ichinose T, Kaun KR, Vogt K, Belliart-Guérin G, Plaçais PY, Robie AA, Nobuhiro Y, Schnaitmann C, Rowell WJ, Johnston RM, Ngo TTB, Chen N, Wyatt K, Nitabach MN, Heberlein U, Thomas P, Branson $\mathrm{KM}$, Tanimoto $\mathrm{H}$, et al. Mushroom body output neurons encode valence and guide memory-based action selection in Drosophila. eLife. 2014 12; 3:e04580. doi: 10.7554/eLife.04580.

Badel L, Ohta K, Tsuchimoto Y, Kazama H. Decoding of Context-Dependent Olfactory Behavior in Drosophila. Neuron. 2016 7; 91:155-67. doi: 10.1016/j.neuron.2016.05.022.

Bates AS, Janssens J, SXE JG, Aerts S. Neuronal cell types in the fly: single-cell anatomy meets single-cell genomics. Curr Opin Neurobiol. 2019 6; 56:125-134. doi: 10.1016/j.conb.2018.12.012.

Bates A, Manton J, Jagannathan S, Costa M, Schlegel P, Rohlfing T, Jefferis G. The natverse, a versatile toolbox for combining and analysing neuroanatomical data. eLife. 2020 4; 9:e53350. doi: 10.7554/eLife.53350.

Bates A, Schlegel P, Roberts R, Drummond N, Tamimi I, Turnbull R, Zhao X, Marin E, Popovici P, Dhawan S, Jamasb A, Javier A, Serratosa CL, Li F, Rubin G, Waddell S, Bock D, Costa M, Jefferis G. Complete Connectomic Reconstruction of Olfactory Projection Neurons in the Fly Brain. Curr Biol. 2020 8; 30:3183-3199.e6. doi: 10.1016/j.cub.2020.06.042.

Berck ME, Khandelwal A, Claus L, Hernandez-Nunez L, Si G, Tabone CJ, Li F, Truman JW, Fetter RD, Matthieu L, Samuel ADT, Cardona A. The wiring diagram of a glomerular olfactory system. eLife. 2016 5; 5:e14859. doi: 10.7554/eLife.14859.

Berni J, Pulver SR, Griffith LC, Bate M. Autonomous Circuitry for Substrate Exploration in Freely Moving Drosophila Larvae. Curr Biol. 2012 10; 22(20):1861-1870. doi: 10.1016/j.cub.2012.07.048.

Bogovic JA, Otsuna H, Heinrich L, Ito M, Jeter J, Meissner G, Nern A, Colonell J, Malkesman O, Ito K, Saalfeld S. An unbiased template of the Drosophila brain and ventral nerve cord. PLOS ONE. 2020 Dec; 15(12):e0236495. doi: 10.1371/journal.pone.0236495.

Boto T, Stahl A, Zhang X, Louis T, Tomchik S. Independent Contributions of Discrete Dopaminergic Circuits to Cellular Plasticity, Memory Strength, and Valence in Drosophila. Cell Rep. 2019 5; 27:2014-2021.e2. doi: 10.1016/j.celrep.2019.04.069.

Bräcker LB, Siju KP, Varela N, Aso Y, Zhang M, Hein I, Vasconcelos ML, Kadow ICG. Essential Role of the Mushroom Body in Context-Dependent CO2 Avoidance in Drosophila. Curr Biol. 2013 7; 23(13):1228-1234. doi: 10.1016/j.cub.2013.05.029.

Budelli G, Ni L, Berciu C, van GL, Knecht Z, Chang E, Kaminski B, Silbering A, Samuel A, Klein M, Benton R, Nicastro D, Garrity P. Ionotropic Receptors Specify the Morphogenesis of Phasic Sensors Controlling Rapid Thermal Preference in Drosophila. Neuron. 2019 2; 101:738-747.e3. doi: 10.1016/j.neuron.2018.12.022.

Cachero S, Gkantia M, Bates AS, Frechter S, Blackie L, McCarthy A, Sutcliffe B, Strano A, Aso Y, Jefferis GSXE. BAcTrace, a tool for retrograde tracing of neuronal circuits in Drosophila. Nat Methods. 2020 Nov; 17(12):12541261. doi: 10.1038/s41592-020-00989-1.

Caron SJC, Ruta V, Abbott LF, Richard A. Random convergence of olfactory inputs in the Drosophila mushroom body. Nature. 2013 5; 497(7447):113-117. doi: 10.1038/nature12063.

Chai PC, Cruchet S, Wigger L, Benton R. Sensory neuron lineage mapping and manipulation in the Drosophila olfactory system. Nat Commun. 2019 2; 10(1):643. doi: 10.1038/s41467-019-08345-4. 
1322

1323

1324

1325

1326

1327

1328

1329

1330

1331

1332

1333

1334

1335

1336

1337

1338

1339

1340

1341

1342

1343

1344

1345

1346

1347

1348

1349

1350

1351

1352

1353

1354

1355

1356

1357

1358

1359

1360

1361

1362

1363

1364

1365

1366

1367

1368

1369

Chiang AS, Lin CY, Chuang CC, Chang HM, Hsieh CH, Yeh CW, Shih CT, Wu J, Wang GT, Yung-Chang C, Wu CC, Chen GY, Ching YT, Lee PC, Lin CY, Lin HH, Chia-Chou W, Hsu HW, Huang YA, Chen JY, et al. Threedimensional reconstruction of brain-wide wiring networks in Drosophila at single-cell resolution. Curr Biol. 2011 1; 21(1):1-11. doi: 10.1016/j.cub.2010.11.056.

Chou YH, Spletter ML, Yaksi E, S LJC, Wilson RI, Luo L. Diversity and wiring variability of olfactory local interneurons in the Drosophila antennal lobe. Nat Neurosci. 2010 4; 13(4):439-449. doi: 10.1038/nn.2489.

Clements J, Dolafi T, Umayam L, Neubarth NL, Berg S, Scheffer LK, Plaza SM. neuPrint: Analysis Tools for EM Connectomics. bioRxiv. 2020 1; doi: 10.1101/2020.01.16.909465.

Coates K, Calle-Schuler S, Helmick L, Knotts V, Martik B, Salman F, Warner L, Valla S, Bock D, Dacks A. The Wiring Logic of an Identified Serotonergic Neuron That Spans Sensory Networks. J Neurosci. 2020 8; 40:6309-6327. doi: 10.1523/JNEUROSCI.0552-20.2020.

Costa M, Manton J, Ostrovsky A, Prohaska S, Jefferis G. NBLAST: Rapid, Sensitive Comparison of Neuronal Structure and Construction of Neuron Family Databases. Neuron. 2016 7; 91:293-311. doi: 10.1016/j.neuron.2016.06.012.

Couto A, Alenius M, Dickson BJ. Molecular, anatomical, and functional organization of the Drosophila olfactory system. Curr Biol. 2005 9; 15(17):1535-1547. doi: 10.1016/j.cub.2005.07.034.

Dolan MJ, Belliart-Guérin G, Shakeel BA, Frechter S, Aurélie LSA, Aso Y, Roberts RJV, Schlegel P, Wong A, Hammad A, Davi B, Rubin GM, Preat T, Pierre-Yves P, Jefferis GSXE. Communication from Learned to Innate Olfactory Processing Centers Is Required for Memory Retrieval in Drosophila. Neuron. 2018 9; 100(3):651-668.e8. doi: 10.1016/j.neuron.2018.08.037.

Dolan MJ, Frechter S, Bates AS, Dan C, Huoviala P, Jv RR, Schlegel P, Dhawan S, Tabano R, Dionne H, Christoforou C, Close K, Sutcliffe B, Giuliani B, Li F, Costa M, Ihrke G, Meissner GW, Bock DD, Aso Y, et al. Neurogenetic dissection of the Drosophila lateral horn reveals major outputs, diverse behavioural functions, and interactions with the mushroom body. eLife. 2019 5; 8:e43079. doi: 10.7554/eLife.43079.

Dorkenwald S, McKellar C, Macrina T, Kemnitz N, Lee K, Lu R, Wu J, Popovych S, Mitchell E, Nehoran B, Jia Z, Bae JA, Mu S, Ih D, Castro M, Ogedengbe O, Halageri A, Ashwood Z, Zung J, Brittain D, et al. FlyWire: Online community for whole-brain connectomics. bioRxiv. 2020 8; doi: 10.1101/2020.08.30.274225.

Dubnau J, Grady L, Kitamoto T, Tully T. Disruption of neurotransmission in Drosophila mushroom body blocks retrieval but not acquisition of memory. Nature. 2001 5; 411(6836):476-480. doi: 10.1038/35078077.

Dweck HKM, Ebrahim SAM, Thoma M, Mohamed AAM, Keesey IW, Trona F, Lavista-Llanos S, Svatoš A, Sachse $\mathrm{S}$, Knaden M, Hansson BS. Pheromones mediating copulation and attraction in Drosophila. Proceedings of the National Academy of Sciences. 2015 May; 112(21):E2829-E2835. doi: 10.1073/pnas.1504527112.

Ebrahim SAM, Dweck HKM, Stökl J, Hofferberth JE, Trona F, Weniger K, Rybak J, Seki Y, Stensmyr MC, Sachse S, Hansson BS, Knaden M. Drosophila Avoids Parasitoids by Sensing Their Semiochemicals via a Dedicated Olfactory Circuit. PLoS Biol. 2015 12; 13(12):e1002318. doi: 10.1371/journal.pbio.1002318.

Eckstein N, Bates AS, Du M, Hartenstein V, Jefferis GSXE, Funke J. Neurotransmitter Classification from Electron Microscopy Images at Synaptic Sites in Drosophila. bioRxiv. 2020 6; doi: 10.1101/2020.06.12.148775.

Egeth M. Behavioral Responses to Light by Headless Anesthetized Drosophila Melanogaster. Perception. 2011 1; 40(2):247-248. doi: 10.1068/p6850.

Eichler K, Li F, Litwin-Kumar A, Youngser P, Andrade I, Schneider-Mizell CM, Saumweber T, Huser A, Eschbach C, Gerber B, Fetter RD, Truman JW, Priebe CE, Abbott LF, Thum AS, Marta Z, Cardona A. The complete connectome of a learning and memory centre in an insect brain. Nature. 2017 8; 548(7666):175-182. doi: 10.1038/nature23455.

Endo K, Aoki T, Yoda Y, Kimura KI, Hama C. Notch signal organizes the Drosophila olfactory circuitry by diversifying the sensory neuronal lineages. Nat Neurosci. 2007 2; 10(2):153-160. doi: 10.1038/nn1832.

Eschbach C, Fushiki A, Winding M, Afonso B, Andrade IV, Cocanougher BT, Eichler K, Gepner R, Si G, ValdesAleman J, Gershow M, Jefferis GS, Truman JW, Fetter RD, Samuel A, Cardona A, Zlatic M. Circuits for integrating learnt and innate valences in the fly brain. bioRxiv. 2020 4; doi: doi.org/10.1101/2020.04.23.058339. 
bioRxiv preprint doi: https://doi.org/10.1101/2020.12.15.401257; this version posted May 14, 2021. The copyright holder for this preprint (which was not certified by peer review) is the author/funder, who has granted bioRxiv a license to display the preprint in perpetuity. It is made available under aCC-BY 4.0 International license.

Felsenberg J, Jacob PF, Walker T, Barnstedt O, Edmondson-Stait AJ, W PM, Otto N, Schlegel P, Sharifi N, Perisse E, Smith CS, Lauritzen JS, Costa M, Jefferis GSXE, Bock DD, Waddell S. Integration of Parallel Opposing Memories Underlies Memory Extinction. Cell. 2018 10; 175(3):709-722.e15. doi: 10.1016/j.cell.2018.08.021.

Fişek M, Wilson RI. Stereotyped connectivity and computations in higher-order olfactory neurons. Nat Neurosci. 2014 2; 17(2):280-288. doi: 10.1038/nn.3613.

Fishilevich E, Vosshall LB. Genetic and functional subdivision of the Drosophila antennal lobe. Curr Biol. 2005 9; 15(17):1548-1553. doi: 10.1016/j.cub.2005.07.066.

Frank DD, Enjin A, Jouandet GC, Zaharieva EE, Para A, Stensmyr MC, Gallio M. Early Integration of Temperature and Humidity Stimuli in the Drosophila Brain. Curr Biol. 2017 8; 27(15):2381-2388.e4. doi: 10.1016/j.cub.2017.06.077.

Frechter S, Bates AS, Tootoonian S, Dolan MJ, Manton J, Jamasb AR, Kohl J, Bock D, Jefferis G. Functional and anatomical specificity in a higher olfactory centre. eLife. 2019 5; 8:e44590. doi: 10.7554/eLife.44590.

Gallio M, Ofstad T, Macpherson L, Wang J, Zuker C. The coding of temperature in the Drosophila brain. Cell. 2011 2; 144:614-24. doi: 10.1016/j.cell.2011.01.028.

Gaudry Q, Hong E, Kain J, de BB, Wilson R. Asymmetric neurotransmitter release enables rapid odour lateralization in Drosophila. Nature. 2013 1; 493:424-428. doi: 10.1038/nature11747.

Grabe V, Baschwitz A, Dweck HKM, Lavista-Llanos S, Hansson BS, Sachse S. Elucidating the Neuronal Architecture of Olfactory Glomeruli in the Drosophila Antennal Lobe. Cell Rep. 2016 9; 16(12):3401-3413. doi: 10.1016/j.celrep.2016.08.063.

Grabe V, Sachse S. Fundamental principles of the olfactory code. Biosystems. 2018 2; 164:94-101. doi: 10.1016/j.biosystems.2017.10.010.

Groschner LN, Miesenböck G. Mechanisms of Sensory Discrimination: Insights from Drosophila Olfaction. Annu Rev Biophys. 2019 5; 48:209-229. doi: 10.1146/annurev-biophys-052118-115655.

Hampel S, McKellar C, Simpson J, Seeds A. Simultaneous activation of parallel sensory pathways promotes a grooming sequence in Drosophila. eLife. 2017 9; 6:e28804. doi: 10.7554/eLife.28804.

Hampel S, Eichler K, Yamada D, Bock DD, Kamikouchi A, Seeds AM. Distinct subpopulations of mechanosensory chordotonal organ neurons elicit grooming of the fruit fly antennae. eLife. 2020 10; 9:e59976. doi: 10.7554/elife.59976.

Heimbeck G, Bugnon V, Gendre N, Keller A, Stocker RF. A central neural circuit for experience-independent olfactory and courtship behavior in Drosophila melanogaster. Proc Natl Acad Sci U S A. 2001 12; 98(26):1533615341. doi: 10.1073/pnas.011314898.

Heisenberg M. Mushroom body memoir: from maps to models. Nat Rev Neurosci. 2003 4; 4:266-75. doi: 10.1038/nrn1074.

Horne J, Langille C, McLin S, Wiederman M, Lu Z, Xu C, Plaza S, Scheffer L, Hess H, Meinertzhagen I. A resource for the Drosophila antennal lobe provided by the connectome of glomerulus VA1v. eLife. 2018 11; 7:e37550. doi: 10.7554/eLife.37550.

Hsu C, Bhandawat V. Organization of descending neurons in Drosophila melanogaster. Sci Rep. 2016 2; 6:20259. doi: 10.1038/srep20259.

Hulse BK, Haberkern H, Franconville R, Turner-Evans DB, Takemura S, Wolff T, Noorman M, Dreher M, Dan C, Parekh R, Hermundstad AM, Rubin GM, Jayaraman V. A connectome of the Drosophila central complex reveals network motifs suitable for flexible navigation and context-dependent action selection. bioRxiv. 2020 12; doi: 10.1101/2020.12.08.413955.

Huoviala P, Dolan MJ, Love FM, Frechter S, Roberts RJV, Mitrevica Z, Schlegel P, Bates AS, Yoshinori A, Rodrigues T, Cornwall H, Marcus S, Bock D, Rubin GM, Costa M, Gregory S X. Neural circuit basis of aversive odour processing in Drosophila from sensory input to descending output. bioRxiv. 2018 8; doi: https://doi.org/10.1101/394403.

Hussain A, Zhang M, Üçpunar HK, Svensson T, Quillery E, Gompel N, Ignell R, Grunwald Kadow IC. Ionotropic Chemosensory Receptors Mediate the Taste and Smell of Polyamines. PLoS Biol. 2016 5; 14(5):e1002454. doi: 10.1371/journal.pbio.1002454. 
Hückesfeld S, Schoofs A, Schlegel P, Miroschnikow A, Pankratz M. Localization of Motor Neurons and Central Pattern Generators for Motor Patterns Underlying Feeding Behavior in Drosophila Larvae. PLoS One. 2015; 10:e0135011. doi: 10.1371/journal.pone.0135011.

Ito M, Masuda N, Shinomiya K, Endo K, Ito K. Systematic analysis of neural projections reveals clonal composition of the Drosophila brain. Curr Biol. 2013 4; 23(8):644-655. doi: 10.1016/j.cub.2013.03.015.

Januszewski M, Kornfeld J, Li P, Pope A, Blakely T, Lindsey L, Maitin-Shepard J, Tyka M, Denk W, Jain V. Highprecision automated reconstruction of neurons with flood-filling networks. Nat Methods. 2018 8; 15:605-610. doi: 10.1038/s41592-018-0049-4.

Jeanne J, Fişek M, Wilson R. The Organization of Projections from Olfactory Glomeruli onto Higher-Order Neurons. Neuron. 2018 6; 98:1198-1213.e6. doi: 10.1016/j.neuron.2018.05.011.

Jefferis GSXE, Potter CJ, Chan AM, Marin EC, Rohlfing T, Maurer CR, Luo L. Comprehensive Maps of Drosophila Higher Olfactory Centers: Spatially Segregated Fruit and Pheromone Representation. Cell. 2007 3; 128(6):1187-1203. doi: 10.1016/j.cell.2007.01.040.

Kadow ICG. State-dependent plasticity of innate behavior in fruit flies. Curr Opin Neurob. 2019 2; 54:60-65. doi: 10.1016/j.conb.2018.08.014.

Knecht Z, Silbering A, Cruz J, Yang L, Croset V, Benton R, Garrity P. Ionotropic Receptor-dependent moist and dry cells control hygrosensation in Drosophila. eLife. 2017 6; 6:e26654. doi: 10.7554/eLife.26654.

Knott G, Marchman H, Wall D, Lich B. Serial section scanning electron microscopy of adult brain tissue using focused ion beam milling. J Neurosci. 2008 3; 28:2959-64. doi: 10.1523/JNEUROSCI.3189-07.2008.

Kohl J, Ostrovsky AD, Frechter S, Jefferis GSXE. A Bidirectional Circuit Switch Reroutes Pheromone Signals in Male and Female Brains. Cell. 2013 12; 155(7):160-23. doi: 10.1016/j.cell.2013.11.025.

Krashes M, Keene A, Leung B, Armstrong J, Waddell S. Sequential use of mushroom body neuron subsets during Drosophila odor memory processing. Neuron. 2007 1; 53:103-15. doi: 10.1016/j.neuron.2006.11.021.

Kurtovic A, Widmer A, Dickson BJ. A single class of olfactory neurons mediates behavioural responses to a Drosophila sex pheromone. Nature. 2007 Mar; 446(7135):542-546. doi: 10.1038/nature05672.

Lacin H, Chen H, Long X, Singer R, Lee T, Truman J. Neurotransmitter identity is acquired in a lineage-restricted manner in the Drosophila CNS. eLife. 2019 3; 8:e43701. doi: 10.7554/eLife.43701.

Lai SL, Awasaki T, Ito K, Lee T. Clonal analysis of Drosophila antennal lobe neurons: diverse neuronal architectures in the lateral neuroblast lineage. Development. 2008 9; 135(17):2883-2893. doi: 10.1242/dev.024380.

Laissue PP, Reiter C, Hiesinger PR, Halter S, Fischbach KF, Stocker RF. Three-dimensional reconstruction of the antennal lobe in Drosophila melanogaster. J Comp Neurol. 1999 3; 405(4):543-552. doi: 10.1002/(SICI)10969861(19990322)405:41\%3C543::AID-CNE71\%3E3.0.CO;2-A.

Lemon RN. Descending Pathways in Motor Control. Annu Rev Neurosci. 2008 7; 31(1):195-218. doi: 10.1146/annurev.neuro.31.060407.125547.

Li F, Lindsey J, Marin E, Otto N, Dreher M, Dempsey G, Stark I, Bates A, Pleijzier M, Schlegel P, Nern A, Takemura S, Eckstein N, Yang T, Francis A, Braun A, Parekh R, Costa M, Scheffer L, Aso Y, et al. The connectome of the adult Drosophila mushroom body provides insights into function. elife. 2020 12; 9:e62576. doi: 10.7554/eLife.62576.

Li PH, Lindsey LF, Januszewski M, Zheng Z, Bates AS, Taisz I, Tyka M, Nichols M, Li F, Perlman E, Maitin-Shepard J, Blakely T, Leavitt L, Jefferis GSXE, Bock D, Jain V. Automated Reconstruction of a Serial-Section EM Drosophila Brain with Flood-Filling Networks and Local Realignment. bioRxiv. 2019 4; doi: 10.1101/605634.

Li Q, Barish S, Okuwa S, Maciejewski A, Brandt AT, Reinhold D, Jones CD, Volkan PC. A Functionally Conserved Gene Regulatory Network Module Governing Olfactory Neuron Diversity. PLOS Genetics. 2016 1; 12(1):e1005780. doi: 10.1371/journal.pgen.1005780.

Liang L, Li Y, Potter C, Yizhar O, Deisseroth K, Tsien R, Luo L. GABAergic projection neurons route selective olfactory inputs to specific higher-order neurons. Neuron. 2013 9; 79:917-31. doi: 10.1016/j.neuron.2013.06.014.

Lovick J, Ngo K, Omoto J, Wong D, Nguyen J, Hartenstein V. Postembryonic lineages of the Drosophila brain: I. Development of the lineage-associated fiber tracts. Dev Biol. 2013 12; 384:228-57. doi: 10.1016/j.ydbio.2013.07.008. 
bioRxiv preprint doi: https://doi.org/10.1101/2020.12.15.401257; this version posted May 14, 2021. The copyright holder for this preprint (which was not certified by peer review) is the author/funder, who has granted bioRxiv a license to display the preprint in perpetuity. It is made available under aCC-BY 4.0 International license.

Mansourian S, Stensmyr MC. The chemical ecology of the fly. Curr Opin Neurobiol. 2015 10; 34:95-102. doi: 10.1016/j.conb.2015.02.006.

Marin EC, Büld L, Theiss M, Sarkissian T, Roberts RJV, Turnbull R, Tamimi IFM, Pleijzier MW, Laursen WJ, Drummond N, Schlegel P, Bates AS, Li F, Landgraf M, Costa M, Bock DD, Garrity PA, Jefferis GSXE. Connectomics analysis reveals first, second, and third order thermosensory and hygrosensory neurons in the adult Drosophila brain. Curr Biol. 2020; 30(16):3167-3182.e4. doi: 10.1016/j.cub.2020.06.028.

McGuire SE, Le PT, Davis RL. The role of Drosophila mushroom body signaling in olfactory memory. Science. 2001 8; 293(5533):1330-1333. doi: 10.1371/journal.pgen.1008963.

Meissner GW, Dorman Z, Nern A, Forster K, Gibney T, Jeter J, Johnson L, He Y, Lee K, Melton B, Yarbrough B, Clements J, Goina C, Otsuna H, Rokicki K, Svirskas RR, Aso Y, Card GM, Dickson BJ, Ehrhardt E, et al. An image resource of subdivided Drosophila GAL4-driver expression patterns for neuron-level searches. bioRxiv. 2020 5; doi: 10.1101/2020.05.29.080473.

Min S, Ai M, Shin Sa, Suh GSB. Dedicated olfactory neurons mediating attraction behavior to ammonia and amines in Drosophila. Proc Natl Acad Sci U S A. 2013; 110(14):E1321-9. doi: 10.1073/pnas.1215680110.

Miroschnikow A, Schlegel P, Schoofs A, Hueckesfeld S, Li F, Schneider-Mizell CM, Fetter RD, Truman JW, Cardona A, Pankratz MJ. Convergence of monosynaptic and polysynaptic sensory paths onto common motor outputs in a Drosophila feeding connectome. eLife. 2018 12; 7:e40247. doi: 10.7554/elife.40247.

Mittal A, Gupta D, Singh A, Lin A, Gupta N. Multiple network properties overcome random connectivity to enable stereotypic sensory responses. Nat Commun. 2020 2; 11:1023. doi: 10.1038/s41467-020-14836-6.

Mohamed AAM, Retzke T, Das Chakraborty S, Fabian B, Hansson BS, Knaden M, Sachse S. Odor mixtures of opposing valence unveil inter-glomerular crosstalk in the Drosophila antennal lobe. Nat Commun. 2019 3; 10(1):1201. doi: 10.1038/s41467-019-09069-1.

Münch D, Galizia C. DoOR 2.0-Comprehensive Mapping of Drosophila melanogaster Odorant Responses. Sci Rep. 2016 2; 6:21841. doi: 10.1038/srep21841.

Namiki S, Dickinson MH, Wong AM, Korff W, Card GM. The functional organization of descending sensorymotor pathways in Drosophila. eLife. 2018 6; 7:e34272. doi: 10.7554/elife.34272.

Nern A, Pfeiffer B, Rubin G. Optimized tools for multicolor stochastic labeling reveal diverse stereotyped cell arrangements in the fly visual system. Proc Natl Acad Sci U S A. 2015 6; 112:E2967-76. doi: 10.1073/pnas.1506763112.

Ni L, Bronk P, Chang E, Lowell A, Flam J, Panzano V, Theobald D, Griffith L, Garrity P. A gustatory receptor paralogue controls rapid warmth avoidance in Drosophila. Nature. 2013 8; 500:580-4. doi: 10.1038/nature12390.

Ohyama T, Schneider-Mizell CM, Fetter RD, Aleman JV, Franconville R, Rivera-Alba M, Mensh BD, Branson KM, Simpson JH, Truman JW, Cardona A, Zlatic M. A multilevel multimodal circuit enhances action selection in Drosophila. Nature. 2015; 520(7549):633-639. doi: 10.1038/nature14297.

Okada R, Awasaki T, Ito K. Gamma-aminobutyric acid (GABA)-mediated neural connections in the Drosophila antennal lobe. J Comp Neurol. 2009 5; 514:74-91. doi: 10.1002/cne.21971.

Olsen SR, Wilson RI. Cracking neural circuits in a tiny brain: new approaches for understanding the neural circuitry of Drosophila. Trends Neurosci. 2008 10; 31(10):512-520. doi: 10.1016/j.tins.2008.07.006.

Otsuna H, Ito M, Kawase T. Color depth MIP mask search: a new tool to expedite Split-GAL4 creation. bioRxiv. 2018 5; doi: 10.1101/318006.

Otto N, Pleijzier M, Morgan I, Edmondson-Stait A, Heinz K, Stark I, Dempsey G, Ito M, Kapoor I, Hsu J, Schlegel P, Bates A, Feng L, Costa M, Ito K, Bock D, Rubin G, Jefferis G, Waddell S. Input Connectivity Reveals Additional Heterogeneity of Dopaminergic Reinforcement in Drosophila. Curr Biol. 2020 8; 30:3200-3211.e8. doi: 10.1016/j.cub.2020.05.077.

Parnas M, Lin AC, Huetteroth W, Miesenböck G. Odor discrimination in Drosophila: from neural population codes to behavior. Neuron. 2013 9; 79(5):932-944. doi: 10.1016/j.neuron.2013.08.006.

Root CM, Masuyama K, Green DS, Enell LE, Nässel DR, Lee CH, Wang JW. A Presynaptic Gain Control Mechanism Fine-Tunes Olfactory Behavior. Neuron. 2008 7; 59(2):311-321. doi: 10.1016/j.neuron.2008.07.003. 
bioRxiv preprint doi: https://doi.org/10.1101/2020.12.15.401257; this version posted May 14, 2021. The copyright holder for this preprint (which was not certified by peer review) is the author/funder, who has granted bioRxiv a license to display the preprint in perpetuity. It is made available under aCC-BY 4.0 International license.

1517

1518

1519

1520

1521

1522

1523

1524

1525

1526

1527

1528

1529

1530

1531

1532

1533

1534

1535

1536

1537

1538

1539

1540

1541

1542

1543

1544

1545

1546

1547

1548

1549

1550

1551

1552

1553

1554

1555

1556

1557

1558

1559

1560

1561

1562

1563

1564

1565

Ryan K, Lu Z, Meinertzhagen I. The CNS connectome of a tadpole larva of Ciona intestinalis (L.) highlights sidedness in the brain of a chordate sibling. eLife. 2016 12; 5:e16962. doi: 10.7554/eLife.16962.

Rybak J, Talarico G, Ruiz S, Arnold C, Cantera R, Hansson B. Synaptic circuitry of identified neurons in the antennal lobe of Drosophila melanogaster. J Comp Neurol. 2016 6; 524:1920-56. doi: 10.1002/cne.23966.

Saalfeld S, Cardona A, Hartenstein V, Tomancak P. CATMAID: collaborative annotation toolkit for massive amounts of image data. Bioinformatics. 2009 8; 25:1984-6. doi: 10.1093/bioinformatics/btp266.

Sayin S, De BJ, Siju K, Wosniack M, Lewis L, Frisch L, Gansen B, Schlegel P, Edmondson-Stait A, Sharifi N, Fisher C, Calle-Schuler S, Lauritzen J, Bock D, Costa M, Jefferis G, Gjorgjieva J, Grunwald KI. A Neural Circuit Arbitrates between Persistence and Withdrawal in Hungry Drosophila. Neuron. 2019 11; 104:544-558.e6. doi: 10.1016/j.neuron.2019.07.028.

Scheffer LK, Xu CS, Januszewski M, Lu Z, Takemura Sy, Hayworth KJ, Huang GB, Shinomiya K, Maitlin-Shepard J, Berg S, Clements J, Hubbard PM, Katz WT, Umayam L, Zhao T, Ackerman D, Blakely T, Bogovic J, Dolafi T, Kainmueller D, et al. A connectome and analysis of the adult Drosophila central brain. eLife. 2020 9; p. e57443. doi: 10.7554/eLife.57443.

Schlegel P, Texada MJ, Miroschnikow A, Schoofs A, Hückesfeld S, Marc P, Schneider-Mizell CM, Lacin H, Li F, Fetter RD, Truman JW, Cardona A, Pankratz MJ. Synaptic transmission parallels neuromodulation in a central food-intake circuit. eLife. 2016 11; 5:e16799. doi: 10.7554/eLife.16799.

Schneider-Mizell CM, Gerhard S, Longair M, Kazimiers T, Li F, Zwart MF, Champion A, Midgley FM, Fetter RD, Saalfeld S, Cardona A. Quantitative neuroanatomy for connectomics in Drosophila. eLife. 2016 3; 5:e12059. doi: 10.7554/eLife.12059.

Séjourné J, Plaçais PY, Yoshinori A, Siwanowicz I, Trannoy S, Thoma V, Tedjakumala SR, Rubin GM, Tchénio P, Ito $\mathrm{K}$, Isabel G, Tanimoto $\mathrm{H}$, Preat T. Mushroom body efferent neurons responsible for aversive olfactory memory retrieval in Drosophila. Nat Neurosci. 2011; 14(7):903-910. doi: 10.1038/nn.2846.

Seki Y, Rybak J, Wicher D, Sachse S, Hansson BS. Physiological and Morphological Characterization of Local Interneurons in the Drosophila Antennal Lobe. J Neurophys. 2010 8; 104(2):1007-1019. doi: 10.1152/jn.00249.2010.

Sen S, Biagini S, Reichert H, VijayRaghavan K. Orthodenticle is required for the development of olfactory projection neurons and local interneurons in Drosophila. Biology Open. 2014 7; 3(8):711-717. doi: 10.1242/bio.20148524.

Shang Y, Claridge-Chang A, Sjulson L, Pypaert M, Miesenböck G. Excitatory Local Circuits and Their Implications for Olfactory Processing in the Fly Antennal Lobe. Cell. 2007 2; 128(3):601-612. doi: 10.1016/j.cell.2006.12.034.

Silbering aF, Rytz R, Grosjean Y, Abuin L, Ramdya P, Jefferis GSXE, Benton R. Complementary Function and Integrated Wiring of the Evolutionarily Distinct Drosophila Olfactory Subsystems. J Neurosci. 2011; 31(38):1335713375. doi: 10.1523/JNEUROSCI.2360-11.2011.

Stensmyr MC, Dweck HKM, Farhan A, Ibba I, Strutz A, Mukunda L, Linz J, Grabe V, Steck K, Lavista-Llanos S, Wicher D, Sachse S, Knaden M, Becher PG, Seki Y, Hansson BS. A Conserved Dedicated Olfactory Circuit for Detecting Harmful Microbes in Drosophila. Cell. 2012 Dec; 151(6):1345-1357. doi: 10.1016/j.cell.2012.09.046.

Stocker RF, Lienhard MC, Borst A, Fischbach KF. Neuronal architecture of the antennal lobe in Drosophila melanogaster. Cell Tissue Res. 1990 10; 262(1):9-34. doi: 10.1016/j.celrep.2016.08.063.

Stocker RF. Drosophila as a focus in olfactory research: Mapping of olfactory sensilla by fine structure odor specificity, odorant receptor expression, and central connectivity. Microsc Res and Tech. 2001; 55(5):284-296. doi: 10.1002/jemt.1178.

Su CY, Menuz K, Carlson JR. Olfactory perception: receptors, cells, and circuits. Cell. 2009 10; 139(1):45-59. doi: 10.1016/j.cell.2009.09.015.

Tanaka N, Endo K, Ito K. Organization of antennal lobe-associated neurons in adult Drosophila melanogaster brain. J Comp Neurol. 2012 12; 520:4067-130. doi: 10.1002/cne.23142.

Tanaka NK, Suzuki E, Dye L, Ejima A, Stopfer M. Dye fills reveal additional olfactory tracts in the protocerebrum of wild-type Drosophila. J Comp Neurol. 2012 12; 520(18):4131-4140. doi: 10.1002/cne.23149. 
bioRxiv preprint doi: https://doi.org/10.1101/2020.12.15.401257; this version posted May 14, 2021. The copyright holder for this preprint (which was not certified by peer review) is the author/funder, who has granted bioRxiv a license to display the preprint in perpetuity. It is made available under aCC-BY 4.0 International license.

1566

1567

1568

1569

1570

1571

1572

1573

1574

1575

1576

1577

1578

1579

1580

1581

1582

1583

1584

1585

1586

1587

1588

1589

1590

1591

1592

1593

1594

1595

1596

1597

1598

1599

1600

1601

1602

1603

1604

Task D, Lin CC, Afify A, Li H, Vulpe A, Menuz K, Potter CJ. Widespread Polymodal Chemosensory Receptor Expression in Drosophila Olfactory Neurons. bioRxiv. 2020; doi: 10.1101/2020.11.07.355651.

Tobin WF, Wilson RI, Lee WCA. Wiring variations that enable and constrain neural computation in a sensory microcircuit. eLife. 2017 5; 6:e24838. doi: 10.7554/eLife.24838.

Truman JW, Bate M. Spatial and temporal patterns of neurogenesis in the central nervous system of Drosophila melanogaster. Dev Biol. 1988; 125(1):145-157. doi: 10.1016/0012-1606(88)90067-x.

Vosshall LB, Wong AM, Axel R. An olfactory sensory map in the fly brain. Cell. 2000 7; 102(2):147-159. doi: 10.1016/s0092-8674(00)00021-0.

Vosshall LB, Stocker RF. Molecular Architecture of Smell and Taste in Drosophila. Annu Rev Neurosci. 2007 Jul; 30(1):505-533. doi: 10.1146/annurev.neuro.30.051606.094306.

Vulpe A, Kim HS, Ballou S, Wu ST, Grabe V, Gonzales CN, Sachse S, Jeanne JM, Su CY, Menuz K. An ammonium transporter is a non-canonical olfactory receptor for ammonia. bioRxiv. 2021 3; doi: 10.1101/2021.03.31.437861.

White J, Southgate E, Thomson J, Brenner S. The structure of the nervous system of the nematode Caenorhabditis elegans. Philos Trans R Soc Lond B Biol Sci. 1986 11; 314:1-340. doi: 10.1098/rstb.1986.0056.

Wilson RI. Early olfactory processing in Drosophila: mechanisms and principles. Annu Rev Neurosci. 2013; 36:217-241. doi: 10.1146/annurev-neuro-062111-150533.

Wilson R, Laurent G. Role of GABAergic inhibition in shaping odor-evoked spatiotemporal patterns in the Drosophila antennal lobe. J Neurosci. 2005 10; 25:9069-79. doi: 10.1523/JNEUROSCI.2070-05.2005.

Wong DC, Lovick JK, Ngo KT, Borisuthirattana W, Omoto JJ, Hartenstein V. Postembryonic lineages of the Drosophila brain: II. Identification of lineage projection patterns based on MARCM clones. Developmental Biology. 2013 Dec; 384(2):258-289. doi: 10.1016/j.ydbio.2013.07.009.

Xu C, Hayworth K, Lu Z, Grob P, Hassan A, García-Cerdán J, Niyogi K, Nogales E, Weinberg R, Hess H. Enhanced FIB-SEM systems for large-volume 3D imaging. eLife. 2017 5; 6:e25916. doi: 10.7554/eLife.25916.

Yu D, Ponomarev A, Davis RL. Altered Representation of the Spatial Code for Odors after Olfactory Classical Conditioning. Neuron. 2004 5; 42(3):437-449. doi: 10.1016/s0896-6273(04)00217-x.

Yu HH, Awasaki T, Schroeder MD, Long F, Yang JS, He Y, Ding P, Kao JC, Wu GYY, Peng H, Myers G, Lee T. Clonal Development and Organization of the Adult Drosophila Central Brain. Curr Biol. 2013 4; 23(8):633-643. doi: 10.1016/j.cub.2013.02.057.

Yu HH, Kao CF, He Y, Ding P, Kao JC, Lee T. A complete developmental sequence of a Drosophila neuronal lineage as revealed by twin-spot MARCM. PLoS Biol. 2010 8; 8(8):e1000461. doi: 10.1371/journal.pbio.1000461.

Zhao B, Sun J, Zhang X, Mo H, Niu Y, Li Q, Wang L, Zhong Y. Long-term memory is formed immediately without the need for protein synthesis-dependent consolidation in Drosophila. Nat Commun. 2019 10; 10(1):4550. doi: 10.1038/s41467-019-12436-7.

Zheng Z, Lauritzen JS, Perlman E, Saalfeld S, Fetter RD, D BCD. A Complete Electron Microscopy Volume of the Brain of Adult Drosophila melanogaster. Cell. 2018; 174:1-14. doi: 10.1016/j.cell.2018.06.019.

Zheng Z, Li F, Fisher C, Ali IJ, Sharifi N, Calle-Schuler S, Hsu J, Masoodpanah N, Kmecova L, Kazimiers T, Perlman E, Nichols M, Li PH, Jain V, Bock DD. Structured sampling of olfactory input by the fly mushroom body. bioRxiv. 2020; doi: 10.1101/2020.04.17.047167. 
Table 3. Description of neuron metadata listed in supplemental files.

\begin{tabular}{|c|c|}
\hline column name & description \\
\hline bodyid & a unique identifier for a single hemibrain neuron \\
\hline pre & the number of presynapses (outputs) a neuron contains, each of these is polyadic \\
\hline post & the number of postsynapses (inputs) to the neuron \\
\hline upstream & the number of incoming connections to a neuron \\
\hline downstream & the number of outgoing connections from a neuron \\
\hline voxels & neuron size in voxels \\
\hline soma & whether the neuron has a soma in the hemibrain volume \\
\hline name & the name of this neuron, as read from neuPrint \\
\hline side & which brain hemisphere contains the neuron's soma \\
\hline connectivity.type & $\begin{array}{l}\text { a subset of neurons within a cell type that share similar connectivity, a connectivity type is dis- } \\
\text { tinguished from a cell type by an ending _letter unless there is only one connectivity type for the } \\
\text { cell type, defined using CBLAST (Scheffer et al., 2020) }\end{array}$ \\
\hline cell.type & $\begin{array}{l}\text { neurons of a shared morphology that take the same cell body fibre tract and come from the } \\
\text { same hemilineage (Bates et al., 2019) }\end{array}$ \\
\hline class & the greater anatomical group to which a neuron belongs, see Figure 1 \\
\hline cellBodyFiber & the cell body fibre for a neuron, as read from neuPrint (Scheffer et al., 2020) \\
\hline ItoLee_Hemilineage & $\begin{array}{l}\text { the hemilineage that we reckon this cell type belongs to, based on expert review of light level } \\
\text { data from the K. Ito and T. Lee groups (Yu et al., 2013, Ito et al., 2013) }\end{array}$ \\
\hline Hartenstein_Hemilineage & $\begin{array}{l}\text { the hemilineage that we reckon this cell type belongs to, based on expert review of light level } \\
\text { data from the V. Hartenstein group (Wong et al., 2013, Lovick et al., 2013) }\end{array}$ \\
\hline putative.classic.transmitter & $\begin{array}{l}\text { putative neurotransmitter based on what neurons in the hemilineage in question have been } \\
\text { shown to express, out of acetylcholine, GABA and/or glutamate }\end{array}$ \\
\hline putative.other.transmitter & potential second neurotransmitter \\
\hline FAFB.match & $\begin{array}{l}\text { the ID of the manual match from the FAFB data set, ID indicates a neuron reconstructed } \\
\text { in FAFBv14 CATMAID, many of these neurons will be available through Virtual Fly Brain, } \\
\text { https://v2.virtualflybrain.org/ }\end{array}$ \\
\hline FAFB.match.quality & $\begin{array}{l}\text { the matcher makers' qualitative assessment of how good this match is: a poor match could be } \\
\text { a neuron from a very similar cell type or a highly untraced neuron that may be the correct cell } \\
\text { type; an okay match should be a neuron that looks to be from the same morphological cell type } \\
\text { but there may be some discrepancies in its arbour; a good match is a neuron that corresponds } \\
\text { well between FAFB and the hemibrain data }\end{array}$ \\
\hline layer & probabilistic mean path length to neuron from ALRNs, depends on connection strengths \\
\hline layer.ct & the mean layer for cell type, rounded to the nearest whole number \\
\hline axon.outputs & number of outgoing connections from the neuron's predicted axon \\
\hline dend.outputs & number of outgoing connections from the neuron's predicted dendrite \\
\hline axon.inputs & number of incoming connections from the neuron's predicted axon \\
\hline dend.inputs & number of incoming connections from the neuron's predicted dendrite \\
\hline total.length & total cable length of the neuron in micrometres \\
\hline axon.length & total axon cable length of the neuron in micrometres \\
\hline dend.length & total dendrite cable length of the neuron in micrometres \\
\hline pd.length & total cable length of the primary dendrite 'linker' between axon and dendrite \\
\hline segregation_index & $\begin{array}{l}\text { a quantification of how polarised a neuron is, in terms of its segregation of inputs onto its pre- } \\
\text { dicted dendrite and outputs onto its axon, where } 0 \text { is no-polarisation and } 1 \text { is totally polarised } \\
\text { (Schneider-Mizell et al., 2016) }\end{array}$ \\
\hline notes & other notes from annotators \\
\hline
\end{tabular}


A

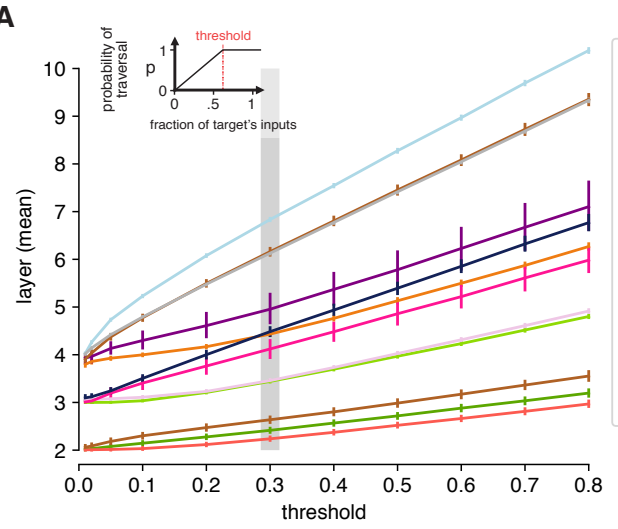

1605

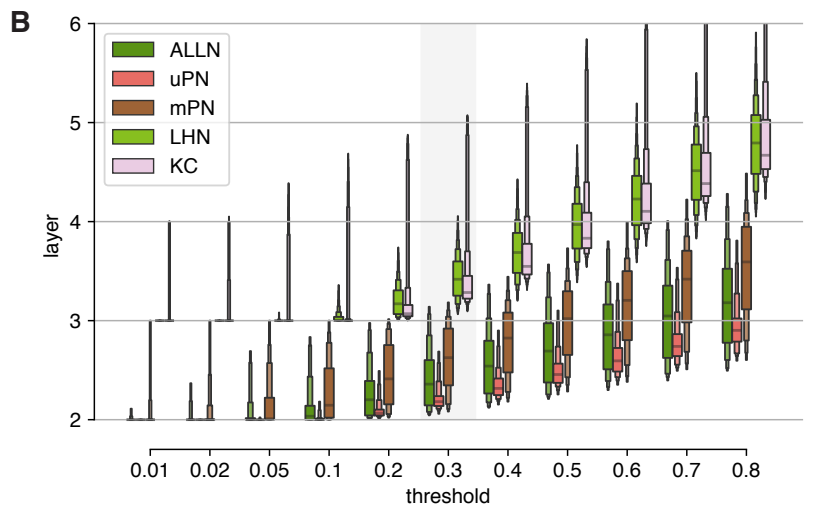

C

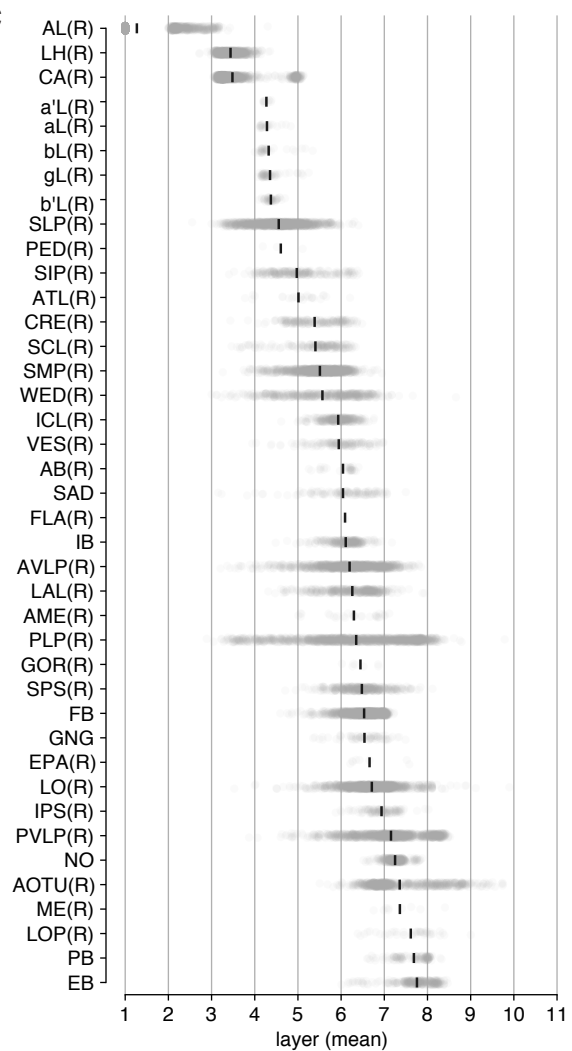

Figure 2-Figure supplement 1. Graph traversal model extended data. A Model parameterization: relative positions are stable across parameter space (with the exception of WEDPNs). Grey bar indicates threshold used for final model (0.3). Error bars represent S.E.M. B Final threshold was chosen using known neuron classes as landmarks. C Mean layer by neuropil. Each neuron is assigned a "primary" neuropil based on where it receives most of its inputs. 
A
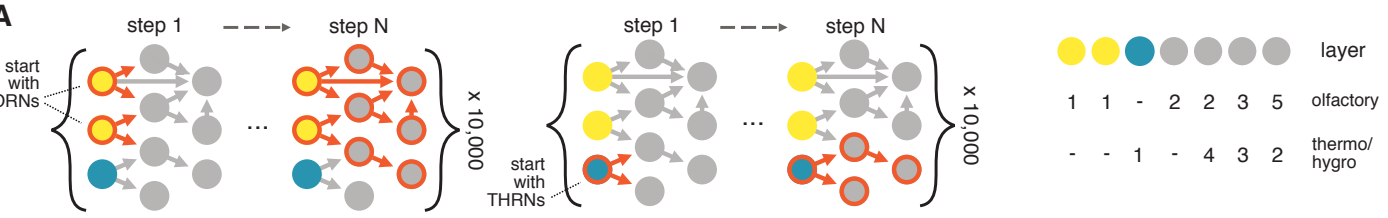

B

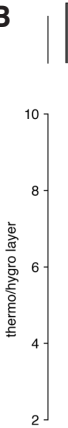

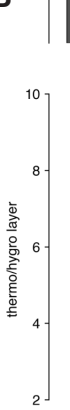
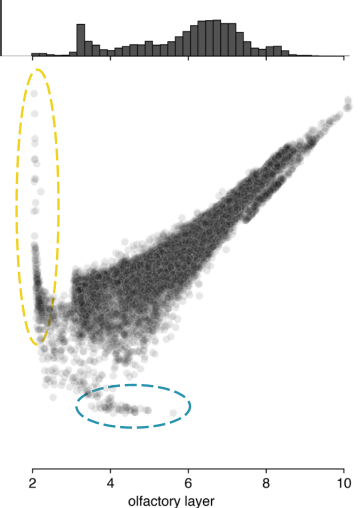

C

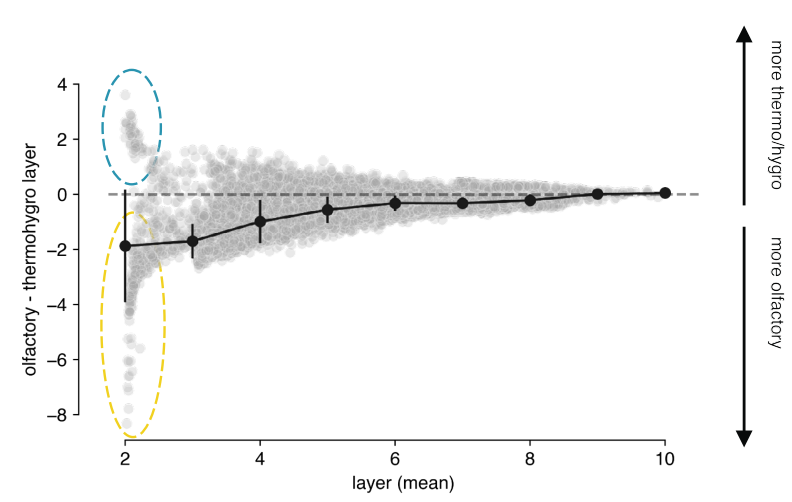

D
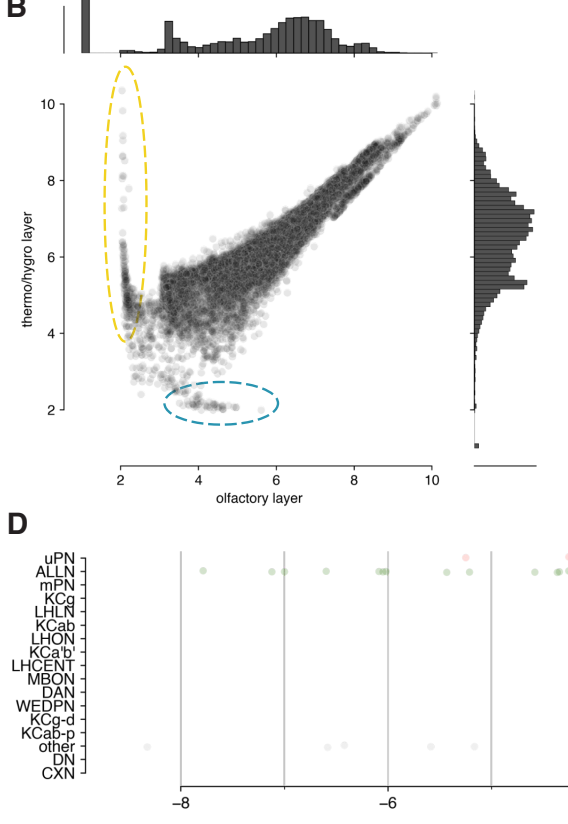

Figure 2-Figure supplement 2. Olfactory vs thermo/hygrosensory layers. A Separate models with olfactory receptor neurons (ORNs) or thermo/hygro-receptor neurons (TRNs/HRNs) as seeds were run to assign layers with respect to the olfactory or thermo/hygrosensory system. B, C Comparison of olfactory vs thermo/hygrosensory layer. Early on there are neurons that appear dedicated to either olfactory (yellow circle) or thermo/hygrosensory (blue circle) sensory information. This separation vanishes in higher layers. Error bars in C represent S.E.M. D Olfactory vs thermo/hygrosensory layer by neuron class. 
bioRxiv preprint doi: https://doi.org/10.1101/2020.12.15.401257; this version posted May 14, 2021. The copyright holder for this preprint (which was not certified by peer review) is the author/funder, who has granted bioRxiv a license to display the preprint in perpetuity. It is made available under aCC-BY 4.0 International license.

A

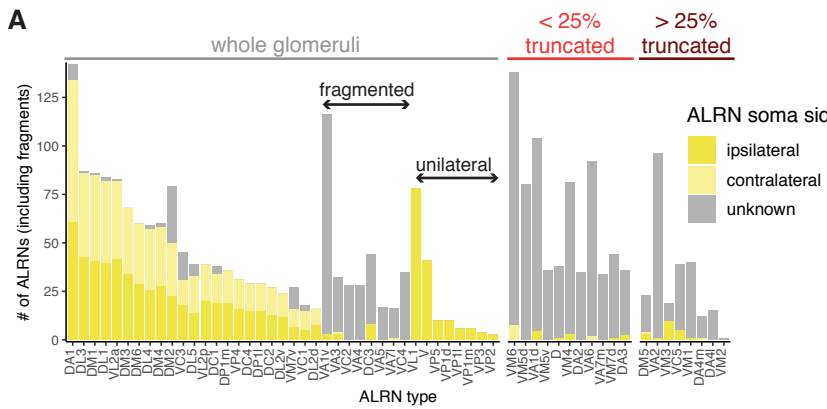

C

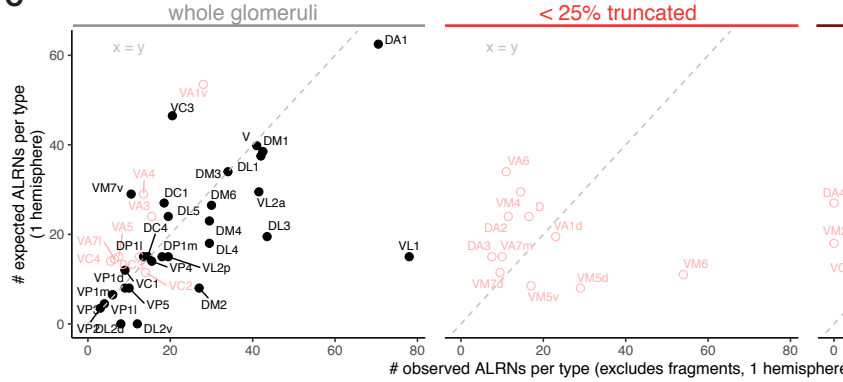

D

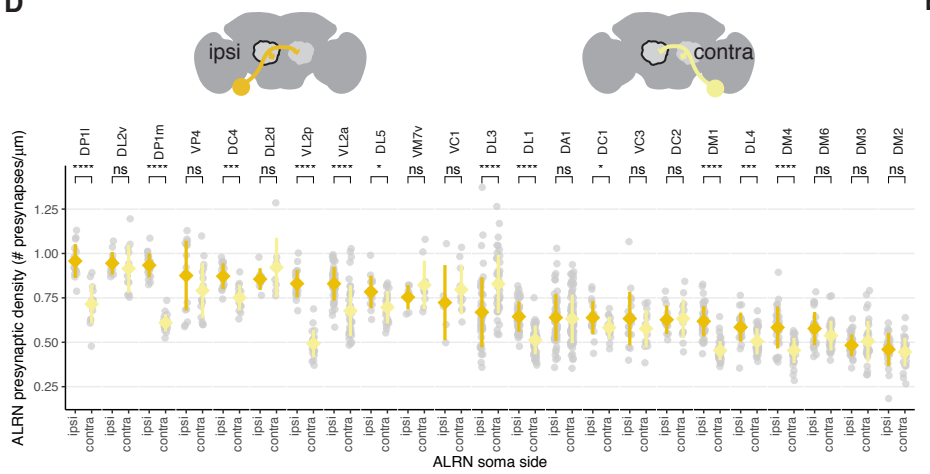

1607

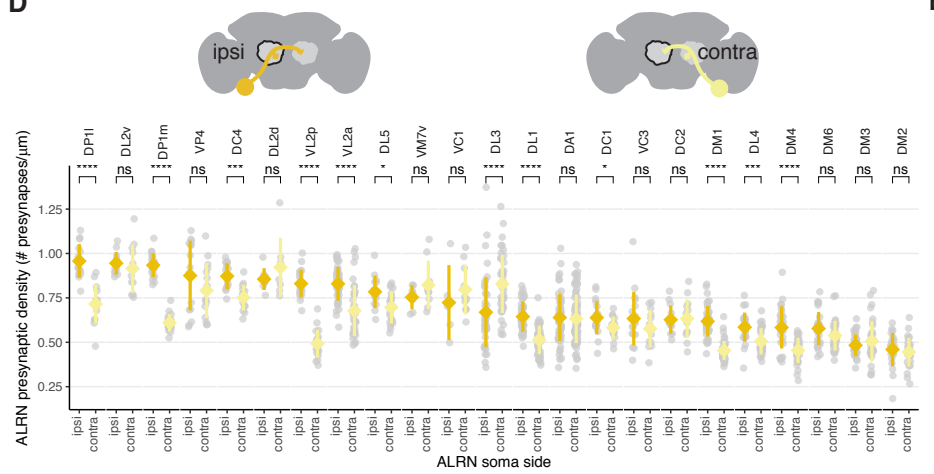

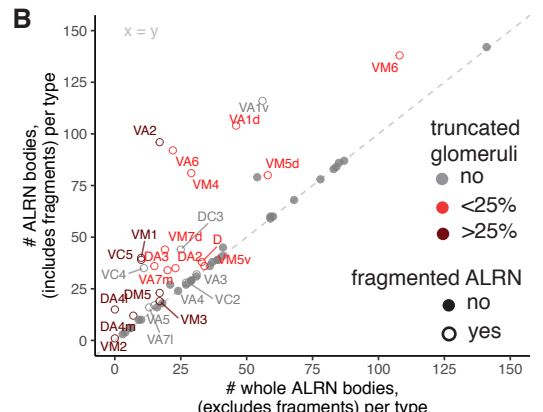

$>25 \%$ truncated

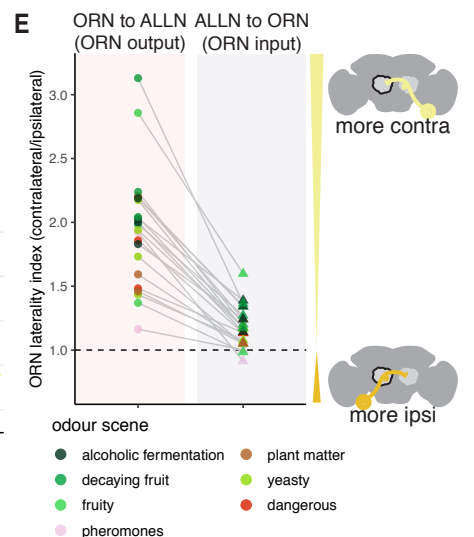

Figure 3-Figure supplement 1. Annotation of ALRN bodies and connectivity features. A Number of unique bodies classified as ALRNs per type and per soma side. Truncated glomeruli $(0,<25 \%$, $>25 \%$ ), fragmented ALRN types in whole glomeruli and unilateral ALRN types are indicated. B Relationship between the number of unique ALRN bodies (including fragments) and whole ALRN bodies (excluding fragments). C Comparison between the number of observed ALRNs (whole) and the expected number per type, in one hemisphere. In A, B and C VM6 ALRNs are plotted as one population, as not every body could be assigned to one of the 3 subpopulations because of the glomerulus truncation.D Presynaptic density for ipsilateral and contralateral ALRNs, per type. Types are ordered by mean ipsilateral density. E Laterality index for ORN and ALLN connectivity (ORN output and ORN input): fraction of contralateral ORN connectivity / fraction of ipsilateral ORN connectivity. Each ORN type is coloured by its functional relevance. Mean comparisons made by Wilcoxon two-sample tests. ns: $p>0.05 ; *$ : $<<=0.05 ; * *: p<=0.01 ; * * *: p<=0.001 ; * * * *: p<=0.0001$ 
bioRxiv preprint doi: https://doi.org/10.1101/2020.12.15.401257; this version posted May 14, 2021. The copyright holder for this preprint (which was not certified by peer review) is the author/funder, who has granted bioRxiv a license to display the preprint in perpetuity. It is made available under aCC-BY 4.0 International license.

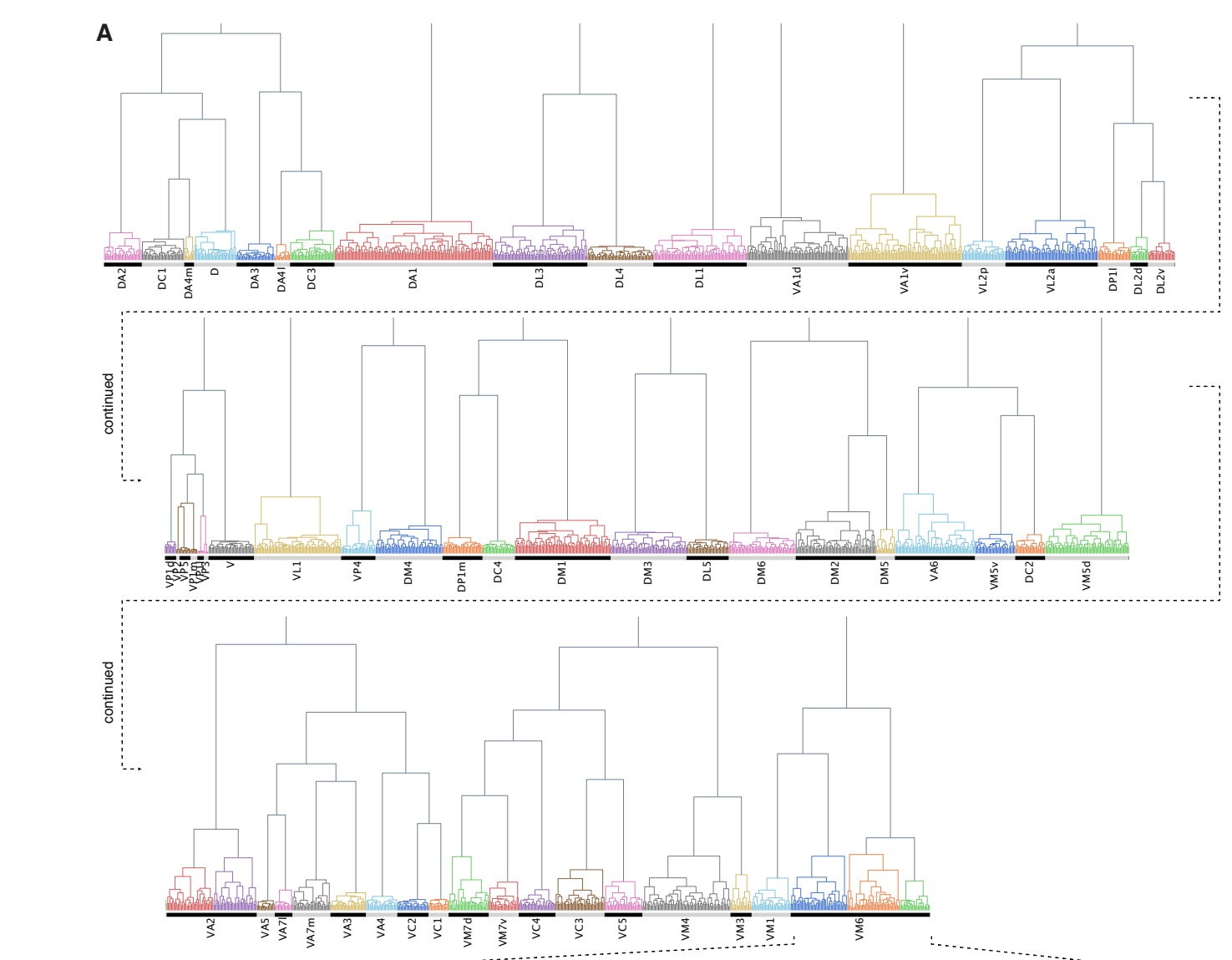

B

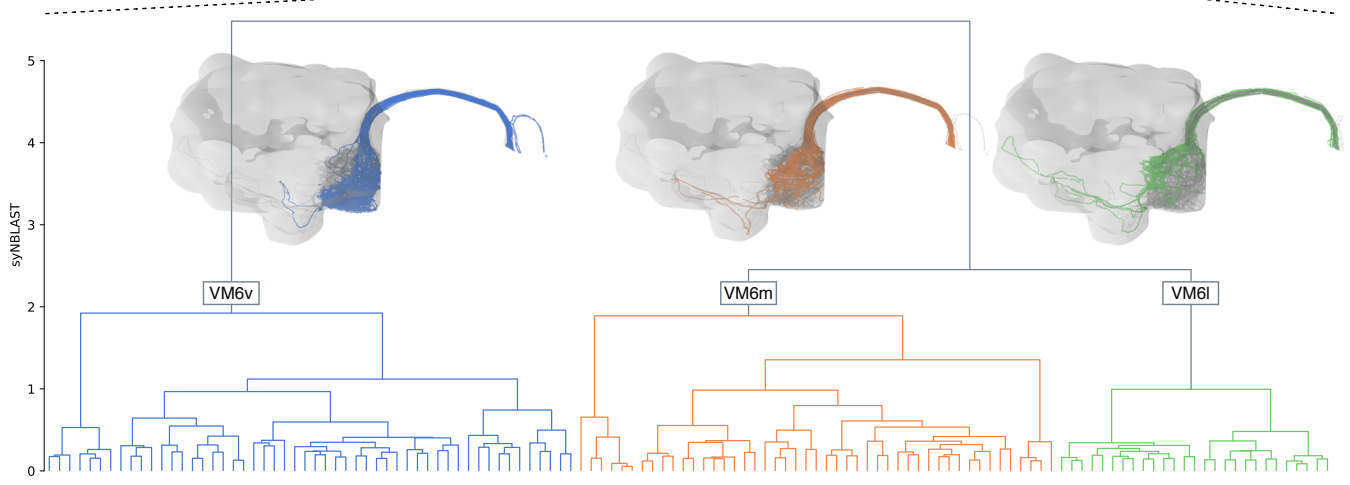

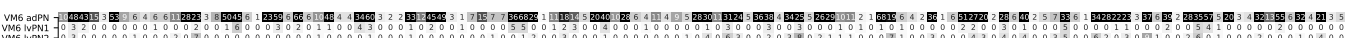

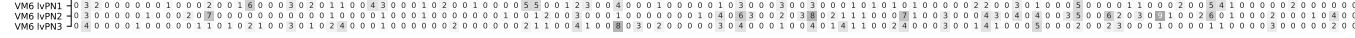

C

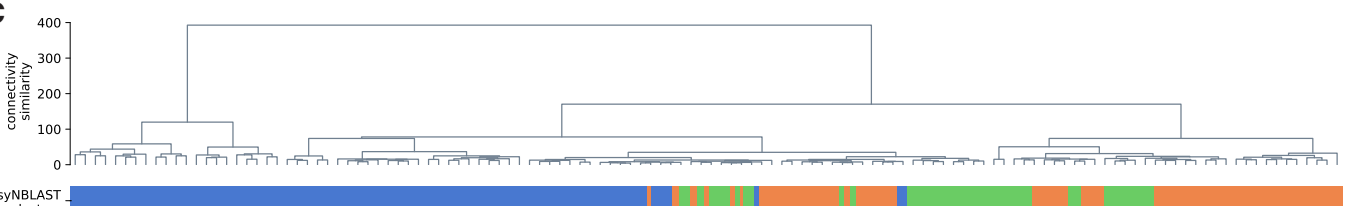

Figure 3-Figure supplement 2. ALRN clustering and subdivision of the VM6 glomerulus. A Synapse-based hierarchical clustering (syNBLAST) of all ALLRNs. B Zoom-in on VM6 ALRNs captures the partition into 3 sub-populations: VM6v, VM6m and VM6I. Heatmap shows connections of VM6 ALRNs onto uniglomerular VM6 ALPNs. C Clustering of VM6 ALRNs based on their downstream connectivity. Color bar at the bottom correspond to syNBLAST clusters in B. The connectivity-based clustering do not align with the subpopulations which suggests that information from the different types of VM6 ALRNs is co-processed by the downstream networks. 


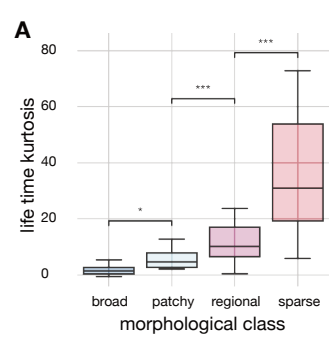

B V glomerulus example

$\mathbf{E}$

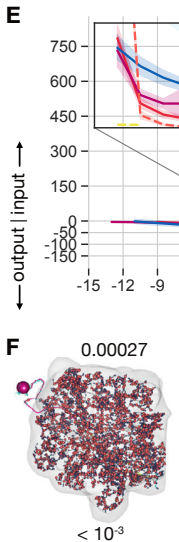

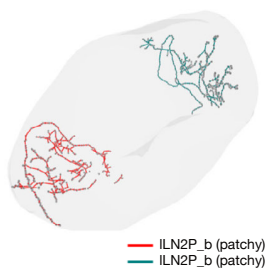

+ sparse rank of glomeruli

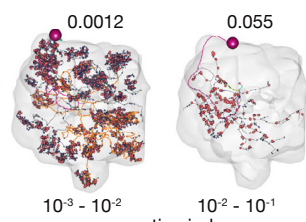

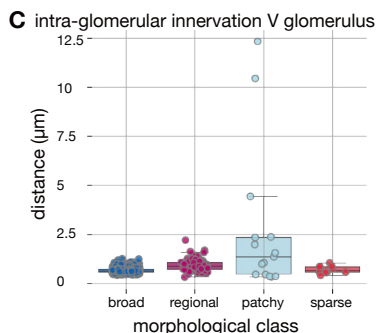

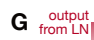

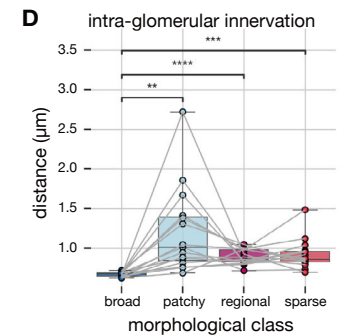

H
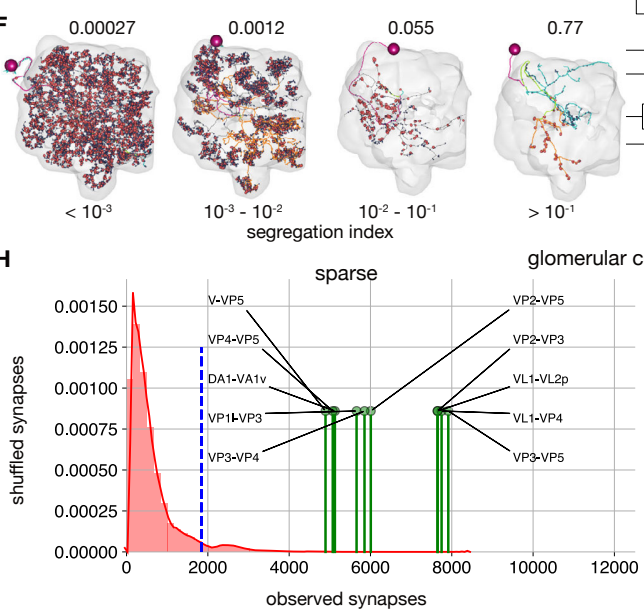

sparse

glomerular co-innervation

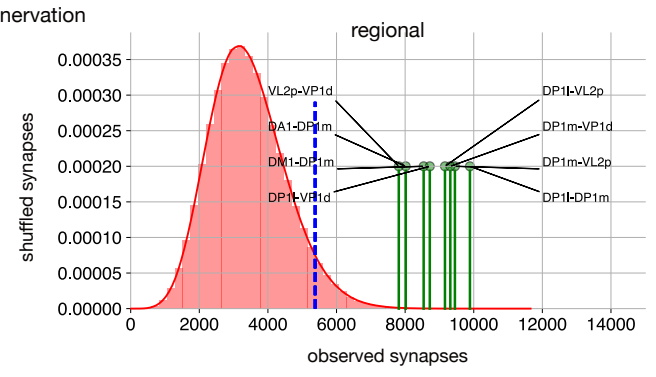

Figure 4-Figure supplement 1. ALLN glomerular innervation patterns. A Sparseness of different ALLNs by morphological lifetime kurtosis class based on glomerular innervation (number of synapses) to calculate the lifetime kurtosis. B Example of two patchy ALLNs that are restricted to different areas of the $V$ glomerulus. C Distances between ALLNs of the same morphology within the $\mathrm{V}$ glomerulus. D Distances between ALLNs of the same morphology in all glomeruli. $\mathbf{E}$ Inputoutput segregation by ALLN types. For each morphological class input and output synapses per glomerulus are plotted in rank order. The inset shows that regional and sparse ALLNs asymptote faster to 0 compared with broad and patchy ALLNs consistent with the selective nature of their inputs. The green line indicates glomerular rank at which at least two of the ALLN types asymptote to 0 . $\mathbf{F}$ Some ALLNs are polarised. The segregation index is a measure of how well they can be split into an axon and a dendrite; the higher the score, the more polarised the neuron.Images show splits for exemplary ALLNs across a range of segregation indices. G Heatmap showing, for all ALLN types with a segregation index above 0.1, their glomerular innervation. For each neuron, for each glomerulus, the proportion of dendritic input synapses is subtracted from the proportion of axonic output synapses in that glomerulus. Negative scores indicate dendritic input, positive ones axonic output. H Glomerular co-innervation per morphological class. Glomeruli that are frequently co-innervated are compared to the random distribution of synapses (in red). The blue dotted line represents the 95th percentile of the distribution of shuffled synapses. Co-innervation of significant pairs of glomeruli for sparse (left) and regional (right) ALLNs. 
bioRxiv preprint doi: https://doi.org/10.1101/2020.12.15.401257; this version posted May 14, 2021. The copyright holder for this preprint (which was not certified by peer review) is the author/funder, who has granted bioRxiv a license to display the preprint in perpetuity. It is made available under aCC-BY 4.0 International license.
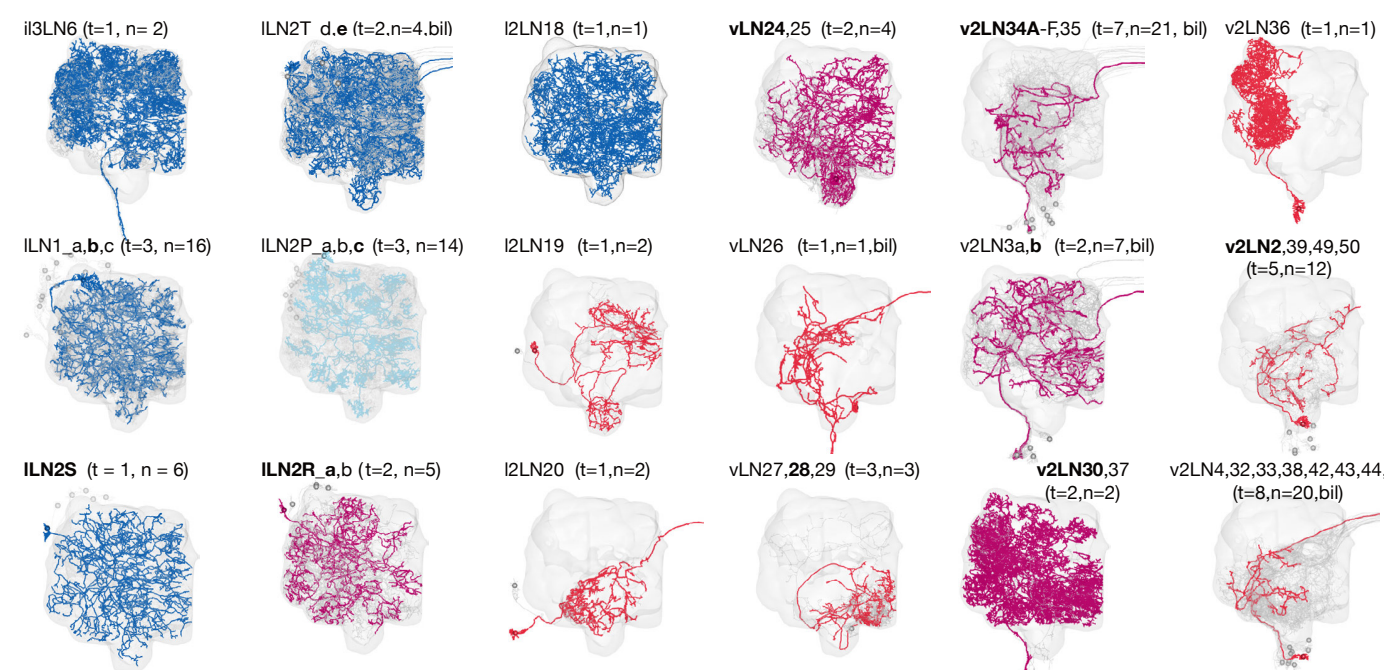

I2LN20 (t=1,n=2)
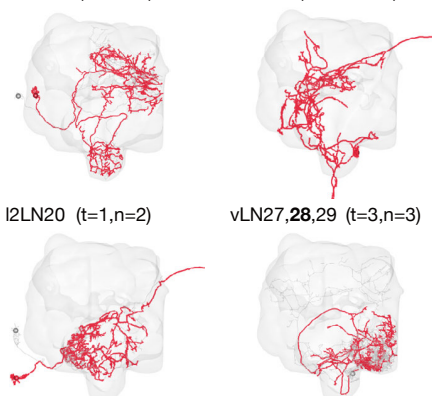

V2LN3a,b (t=2,n=7,bil)

V2LN2,39,49,50

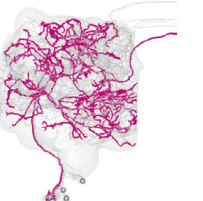
$(\mathrm{t}=5, \mathrm{n}=12)$
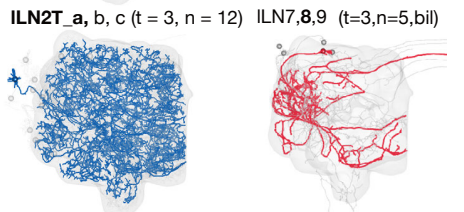

ILN10-17 (t=10,n=29)
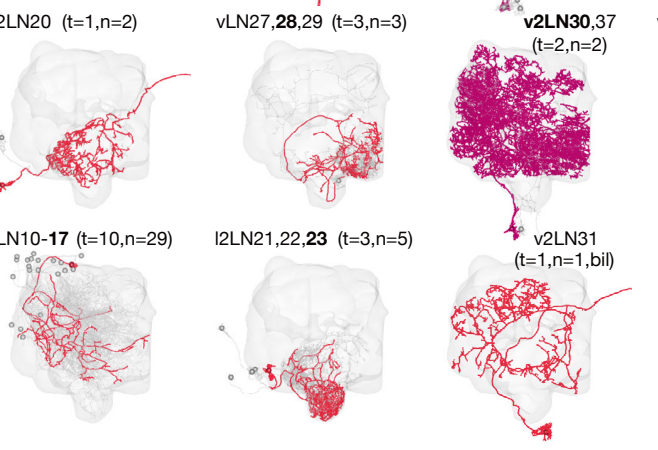

v2LN4,32,33,38,42,43,44,45 $(\mathrm{t}=8, \mathrm{n}=20$, bil)

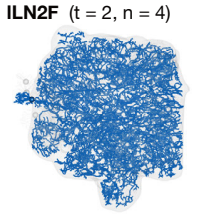

broad $\bullet$ regional patchy sparse $\mathrm{t}=$ number of types $\mathrm{n}=$ number of neurons

Figure 5-Figure supplement 1. Antennal lobe local neuron groups. ALLN types can be grouped into 25 anatomical groups that differ in their lineage, morphology, area of innervation and density of innervation. One neuron is plotted in colour as an example, the remaining are in grey. For groups with more than one type, the type of the coloured neuron is in bold. The group V2LN34AF, 35 includes regional and sparse types. Note that each of the ILN2T_d extends several neurites towards the midline. bil $=$ neurons project bilaterally. 
bioRxiv preprint doi: https://doi.org/10.1101/2020.12.15.401257; this version posted May 14,2021 . The copyright holder for this preprint (which was not certified by peer review) is the author/funder, who has granted bioRxiv a license to display the preprint in perpetuity. It is made available under aCC-BY 4.0 International license.

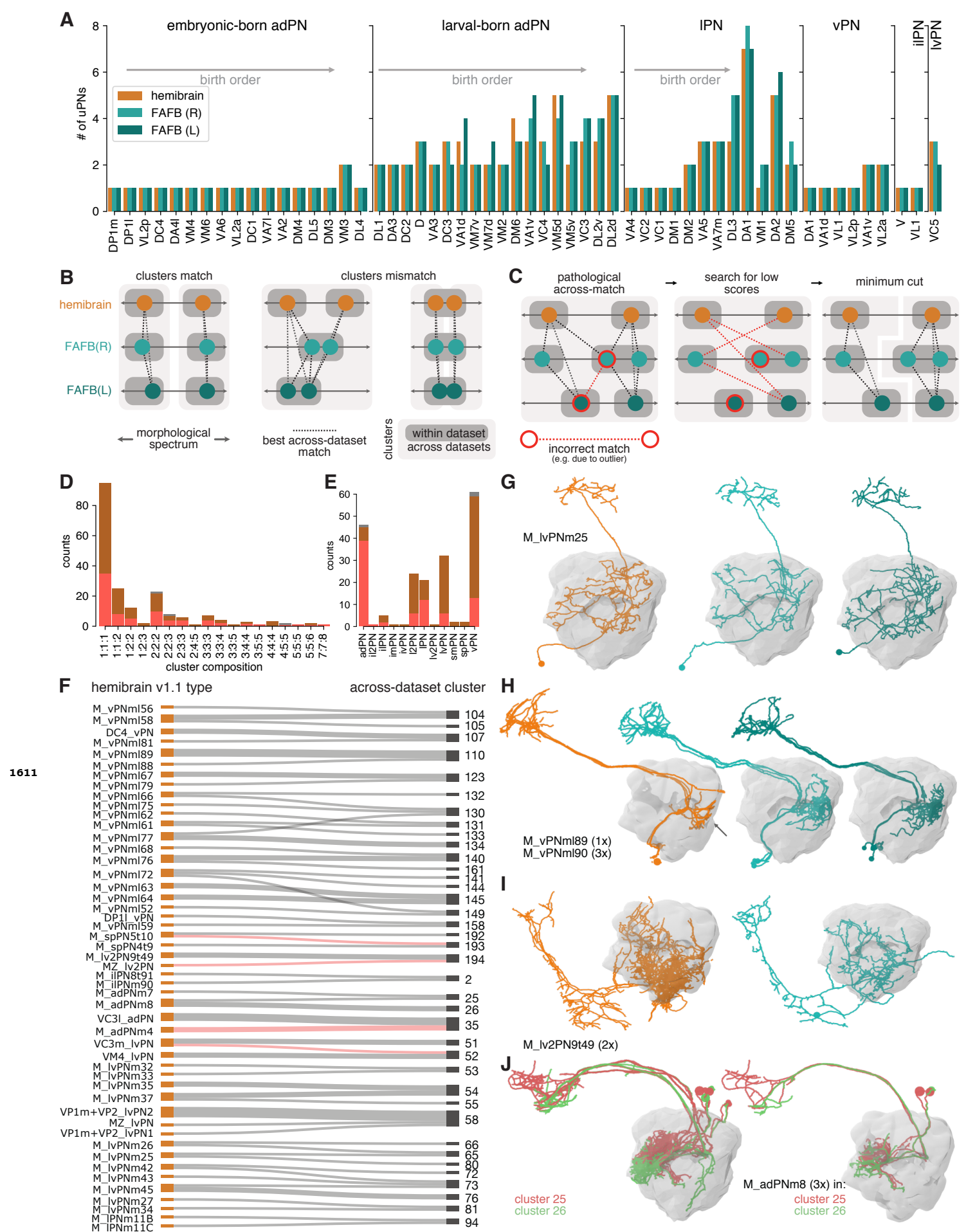

Figure 6-Figure supplement 1. Comparison of ALPNs across three hemispheres. A Counts for 56 uPN types across the three hemispheres. If known, order corresponds to order of birth. B Expanded explanation for across-dataset clustering. C Illustration of refinement of initial clusters to deal with incorrect "pathological" (red outline) across-dataset matches. D Cluster composition as number of neurons from the three datasets. $\mathbf{E}$ Number of clusters per ALPN lineage. $\mathbf{F}$ Flow diagram for hemibrain v1.1 types that are merged or shuffled in the across-dataset clusters. Across-dataset merges identified as wrong are highlighted in red (see I for example). G-J Illustrative examples. G Single mPN that can be tracked 1:1:1 across datasets. $\mathbf{H}$ Truncated (arrow) hemibrain mPNs matched to FAFB ALPNs. I mPN without a match in FAFB(L) caused an incorrect merge into cluster 194. J M_adPNm8 mPNs are split into across-dataset clusters 25 and 26. 
A

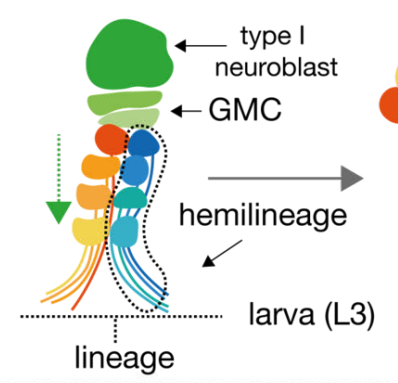

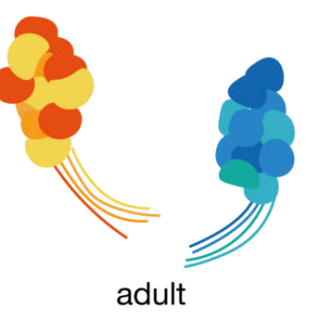

adult
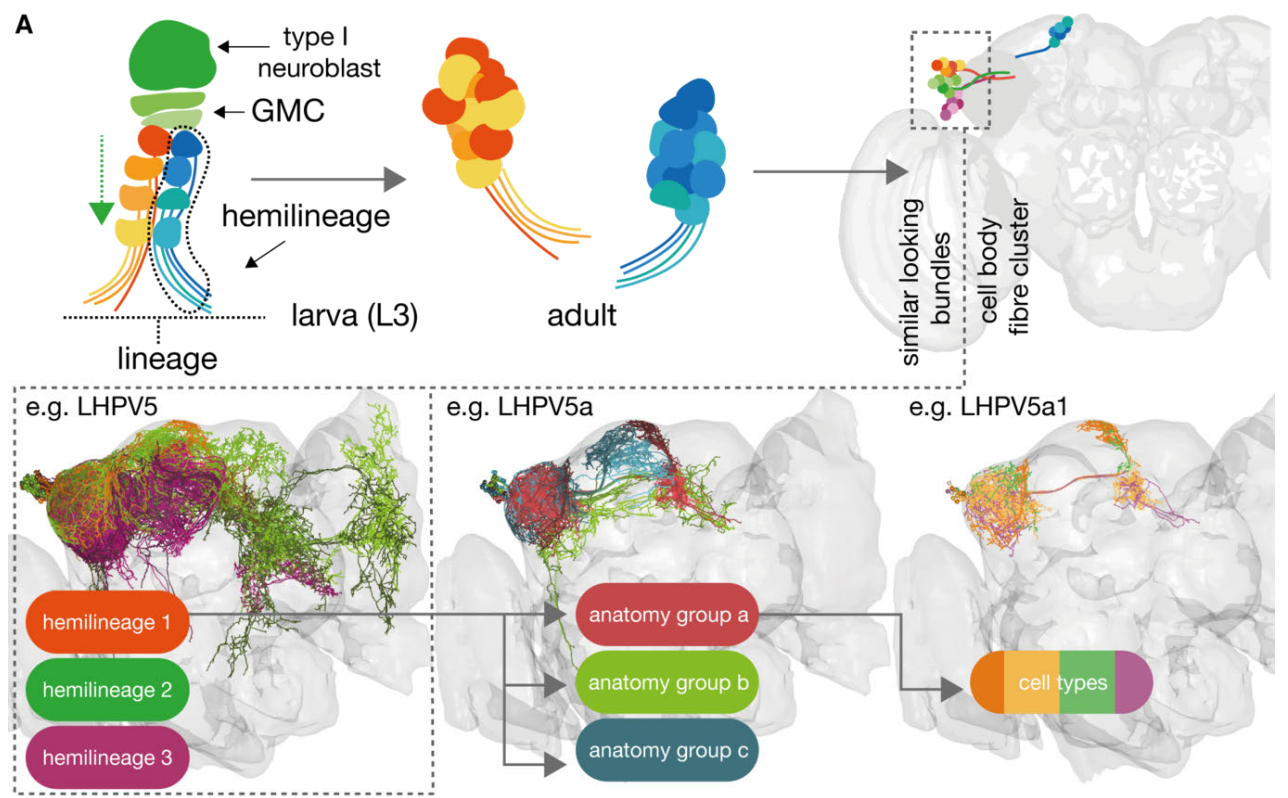

B hemilineage

1612
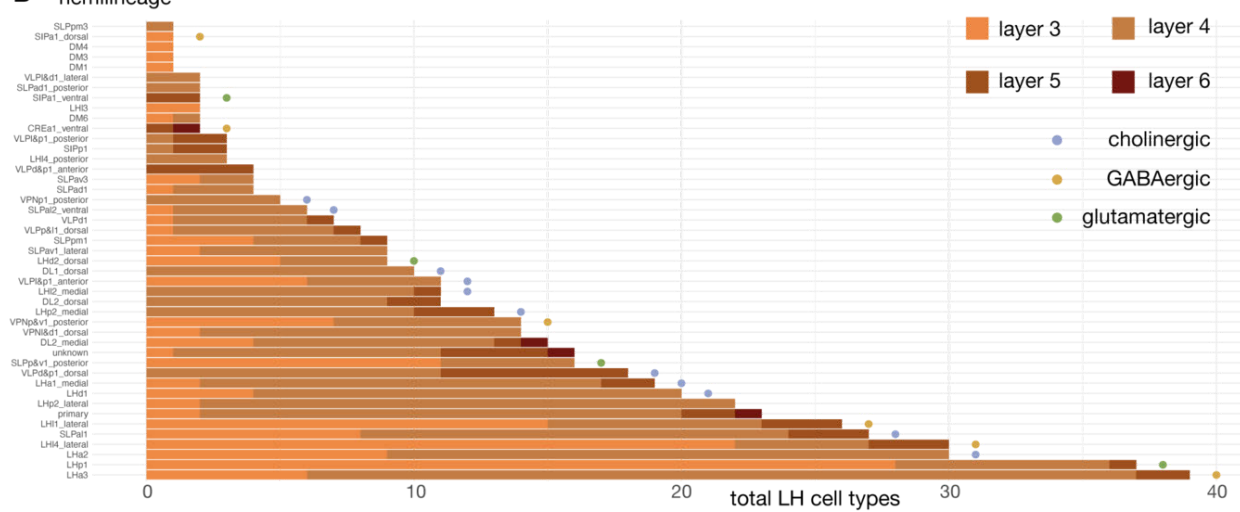

Figure 8-Figure supplement 1. Defining cell types for third-order olfactory neurons. The scheme by which we have named LHNs derives from the system we implemented in Frechter et al. (2019). A Similar-looking hemilineages are grouped together, neurons of similar coarse morphology are grouped together into 'anatomy groups' and each anatomy group is broken down into approximately isomorphic cell type (Bates et al., 2019). B The number of LHN cell types contributed by different hemilineages, which approximate cell body fibre tracts (Wong et al., 2013; Lovick et al., 2013). Names from the scheme by the K. Ito and T. Lee groups ( $Y u$ et al., 2013; Ito et al., 2013). Colours give a breakdown by their layer. Putative transmitter indicated by coloured circles. 

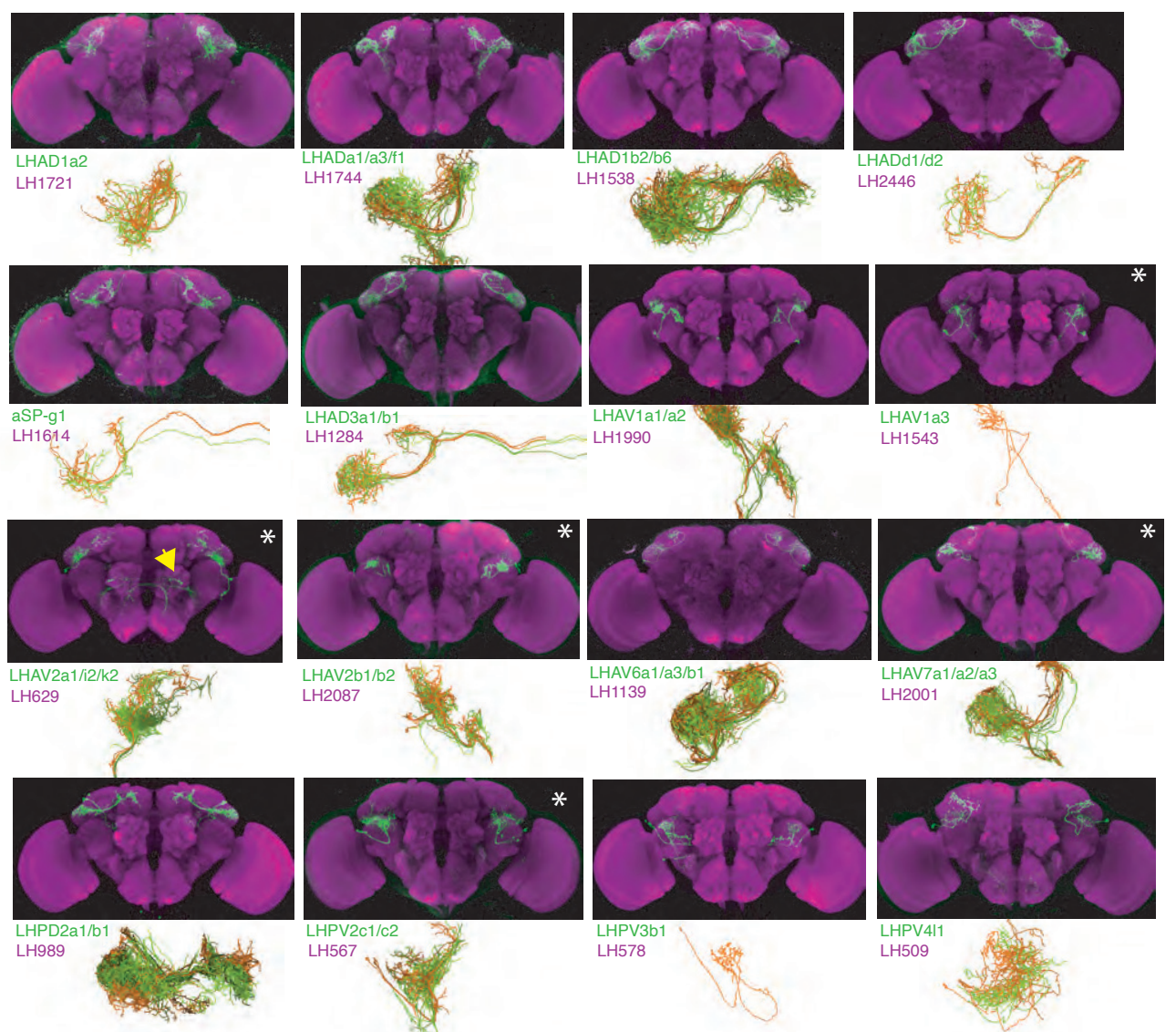

1613
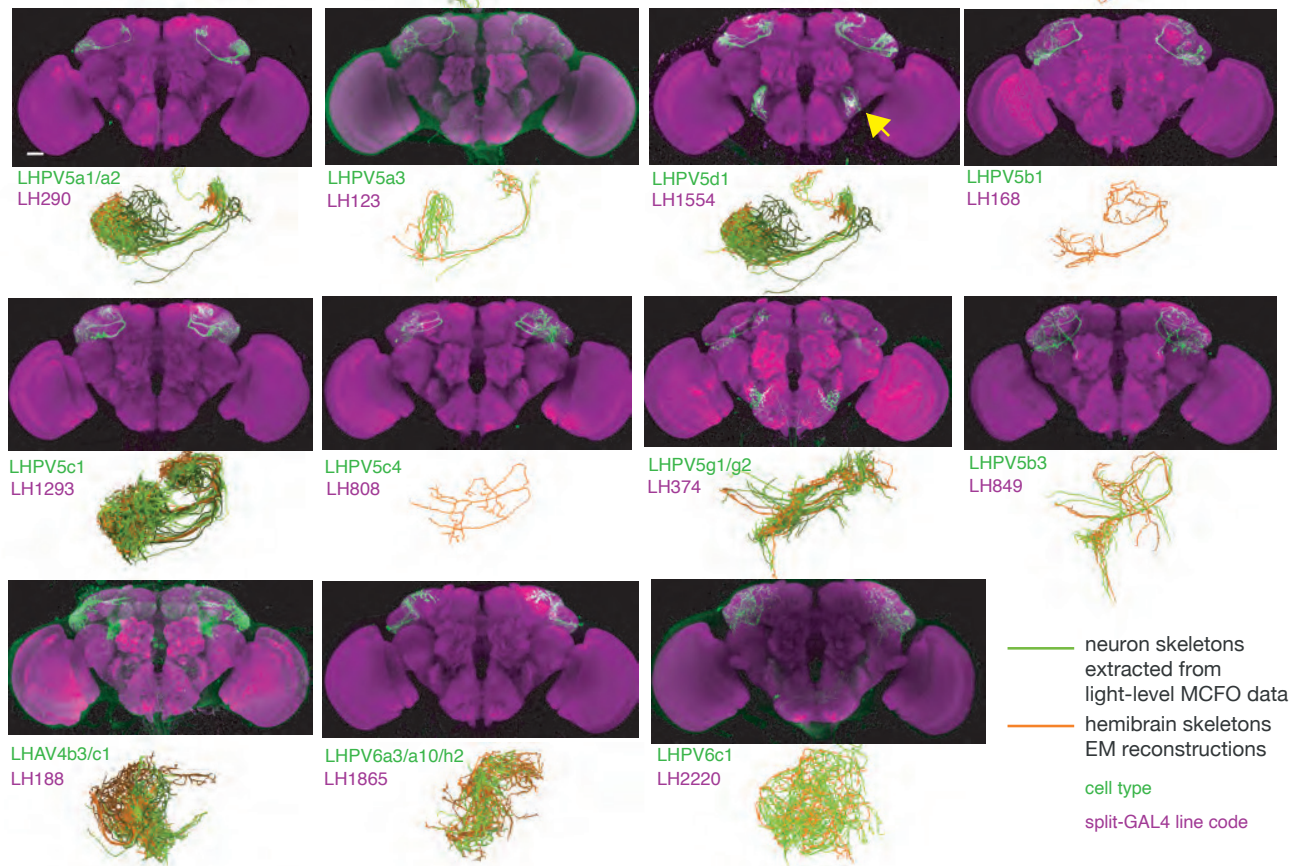

H849
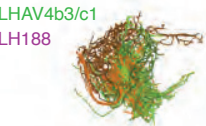

Figure 8-Figure supplement 2. Split-GAL4 lines for excitatory lateral horn output neurons.

Putative excitatory output neurons of the lateral horn for which there are targeted genetic reagents as well as EM reconstructions (Dolan et al., 2019; Bates et al., 2020b; Scheffer et al., 2020). Expression of split-GAL4 lines are visualised using UAS-csChrimson::mVenus in attP18 (green), with nc82 as a neuropil stain (magenta) (Dolan et al., 2019). Off-target expression in the brain for non-ideal lines labelled with a yellow arrow. See www.janelia.org/split-gal4 for image data. 


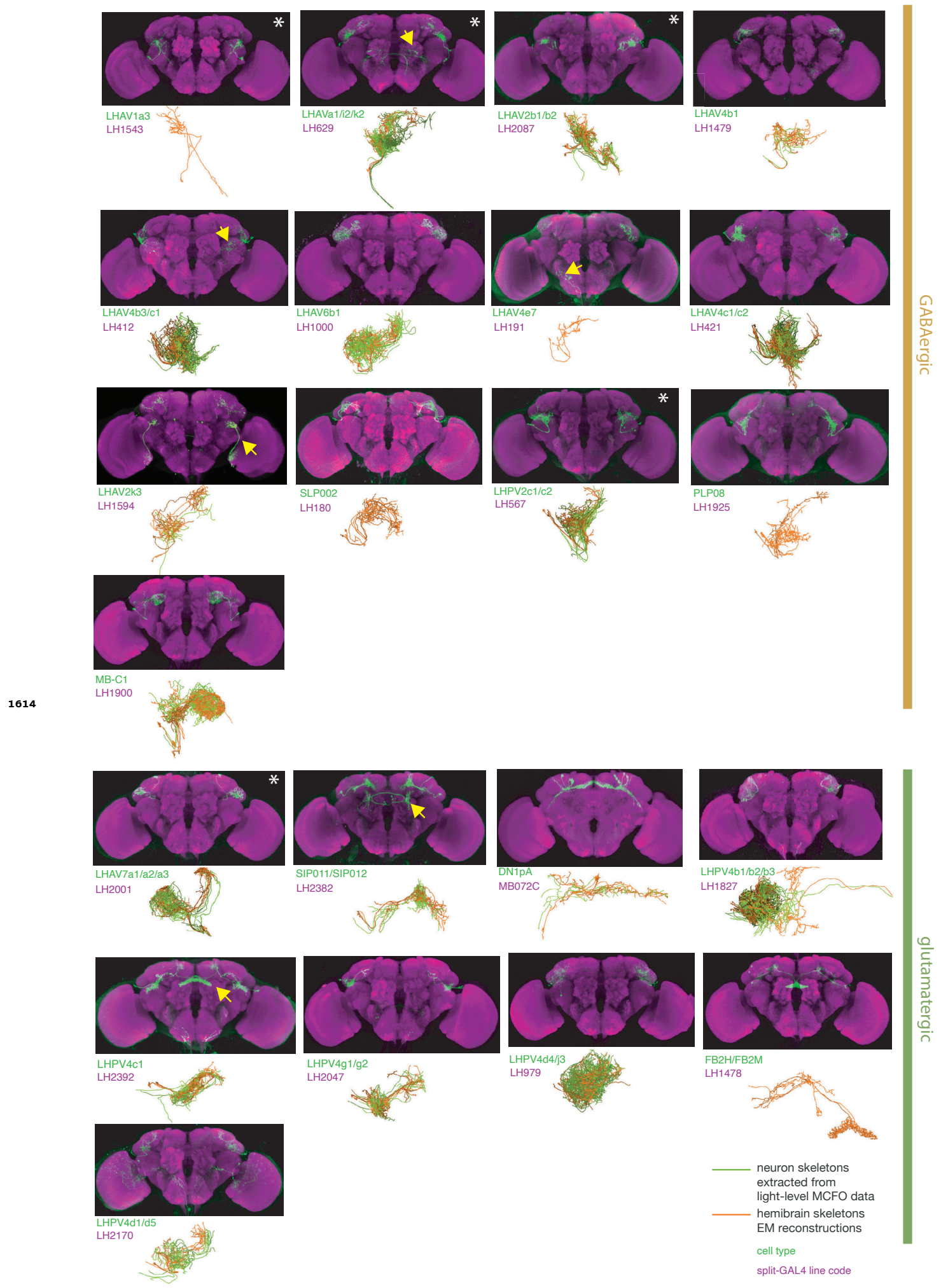

Figure 8-Figure supplement 3. Split-GAL4 lines for inhibitory lateral horn output neurons. Putative inhibitory output neurons of the lateral horn for which there are targeted genetic reagents as well as EM reconstructions (Dolan et al., 2019; Scheffer et al., 2020; Bates et al., 2020b). See www.janelia.org/split-gal4 for image data. 


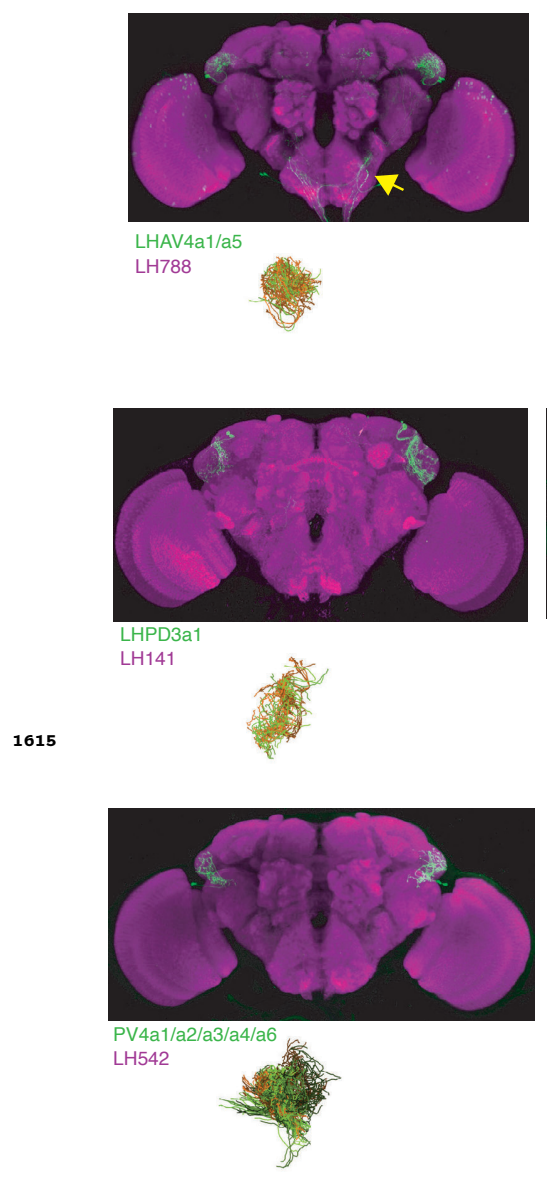

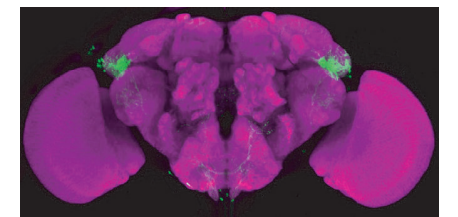

AV4a4/g $2 / g 11 / g 15$ LH247
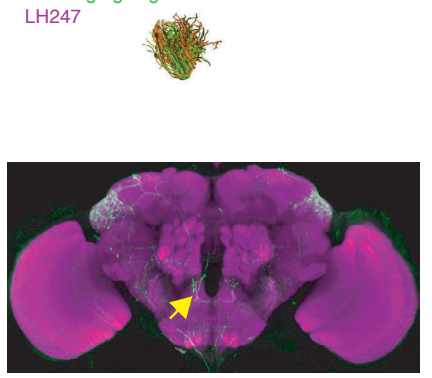

LHPD3a2/a4 LH2385

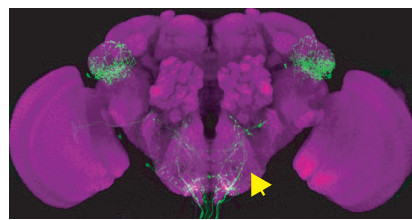

PV4a11/a12/j1/j2

LH1893

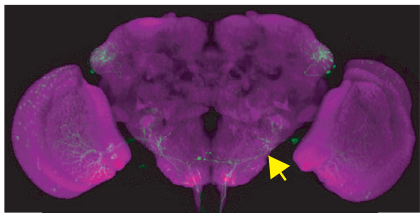

LHPV4a7 LH2223

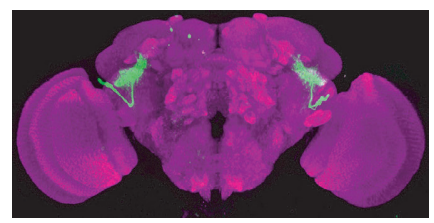

LHPV2a1/a2 LH258
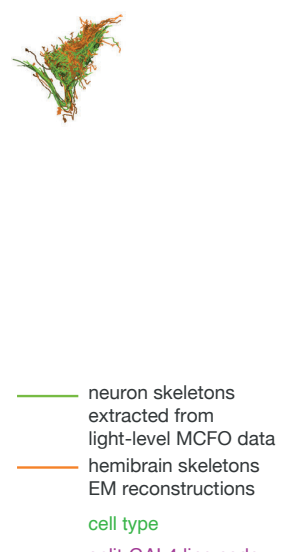

split-GAL4 line code

Figure 8-Figure supplement 4. Split-GAL4 lines for lateral horn local neurons. Putative local neurons of the lateral horn for which there are targeted genetic reagents as well as EM reconstructions (Dolan et al., 2019; Bates et al., 2020b; Scheffer et al., 2020). See www.janelia.org/split-gal4 for image data. 


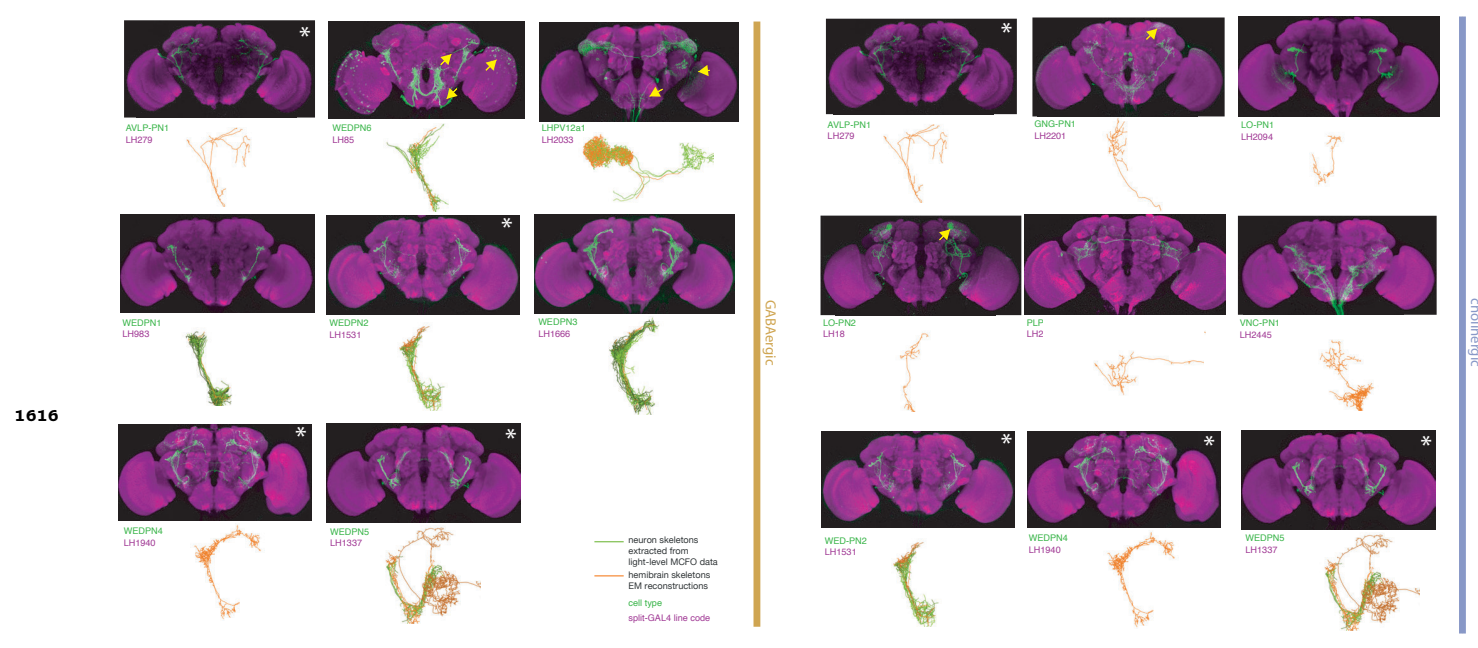

Figure 8-Figure supplement 5. Split-GAL4 lines for lateral horn input neurons. Putative non-olfactory input neurons to the lateral horn for which there are targeted genetic reagents as well as EM reconstructions (Dolan et al., 2019; Scheffer et al., 2020; Bates et al., 2020b). See www.janelia.org/split-gal4 for image data. 
A

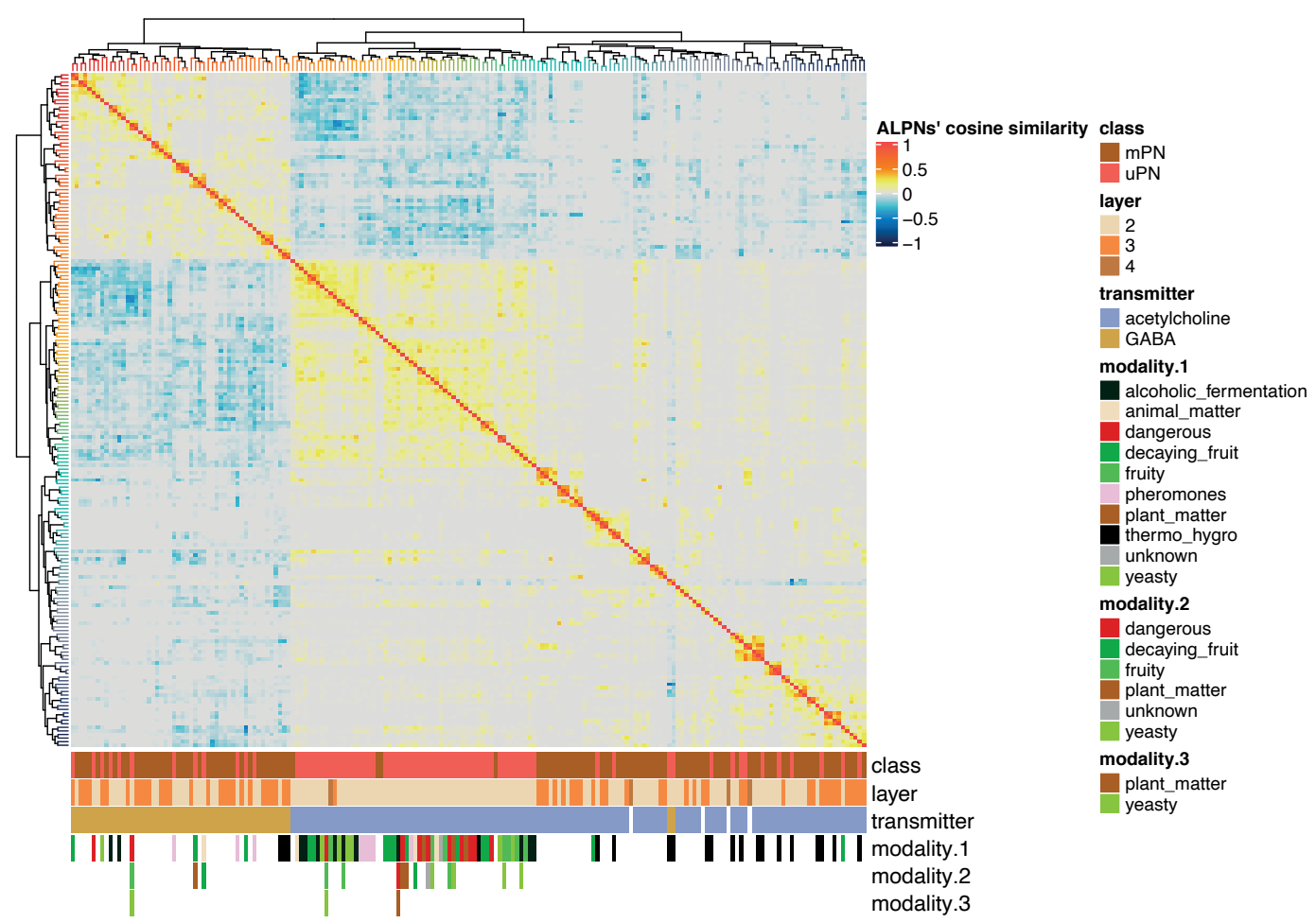

B

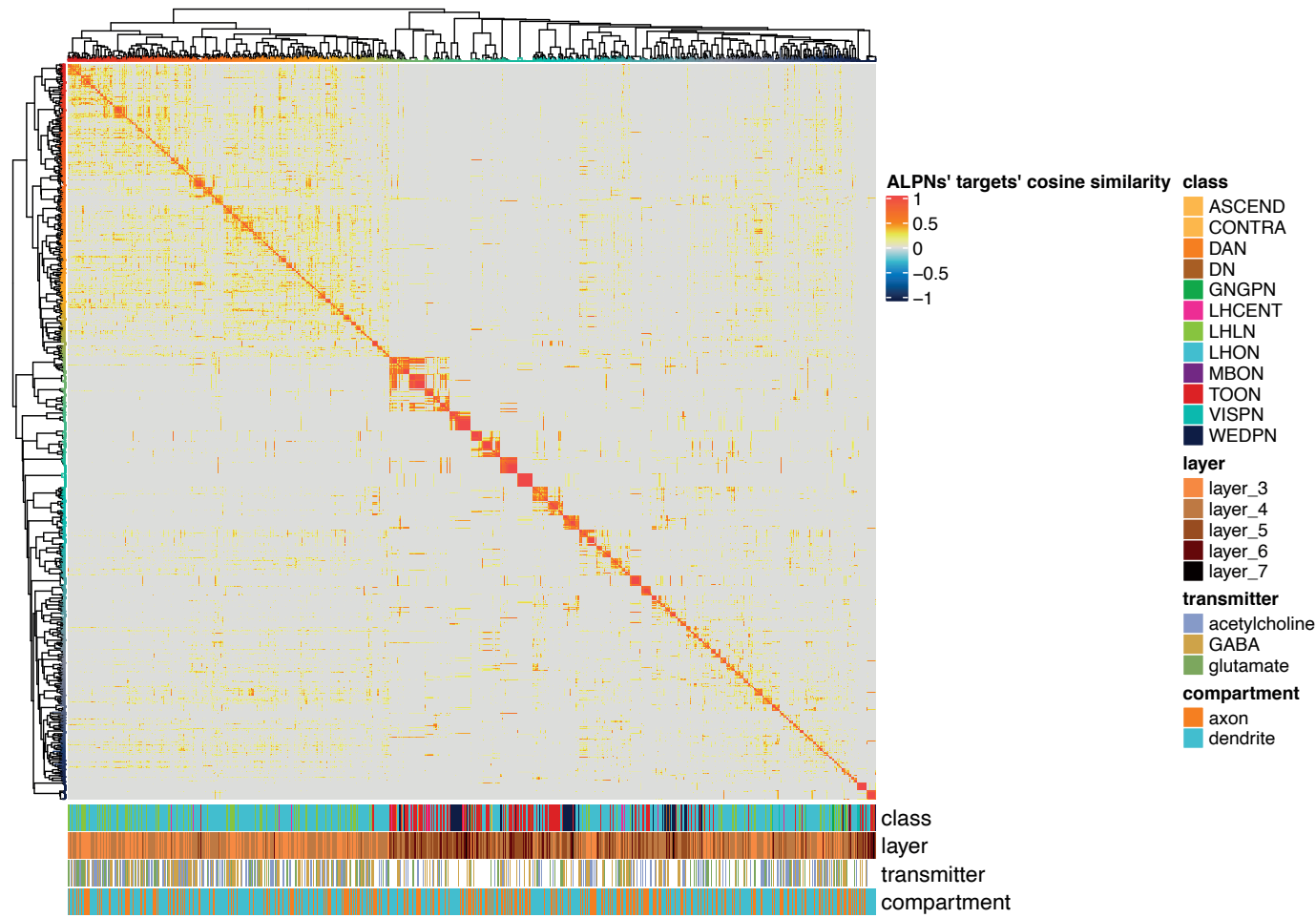

Figure 9-Figure supplement 1. Neurons at the ALPN axon $\rightarrow$ target connection, clustered by connection similarity. A Cosine similarity calculated between ALPN cell types, based on ALPN $\rightarrow$ targets connection strengths, see Figure 9. B Cosine similarity calculated between ALPN' target connectivity types, broken into axon and dendrite and based on ALPN $\rightarrow$ targets connection strengths. Clustering by Ward's method, method 'ward.D2' with the base R function hclust. 
bioRxiv preprint doi: https://doi.org/10.1101/2020.12.15.401257; this version posted May 14, 2021. The copyright holder for this preprint (which was not certified by peer review) is the author/funder, who has granted bioRxiv a license to display the preprint in perpetuity. It is made available under aCC-BY 4.0 International license.

A whole class connectivity

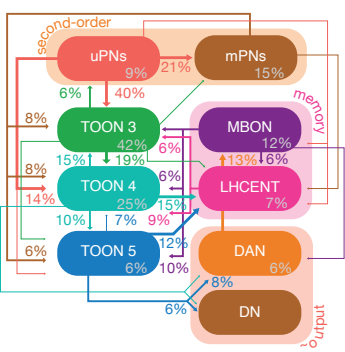

c axo-axonic connectivity

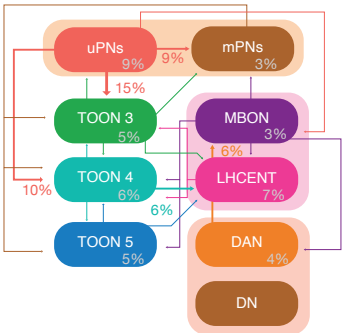

E dendro-axonic connectivity

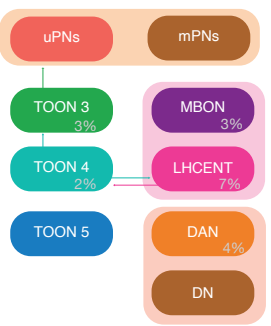

G GABAergic connectivity

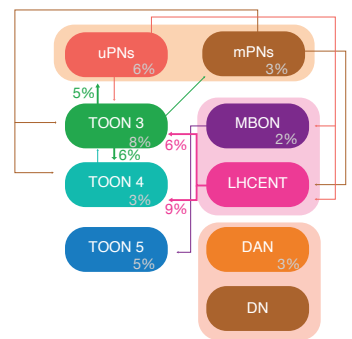

B axo-dendritic connectivity
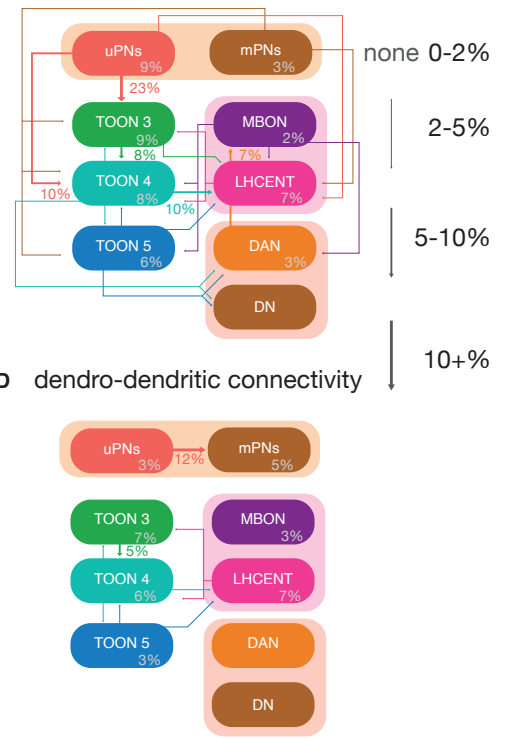

F cholinergic connectivity

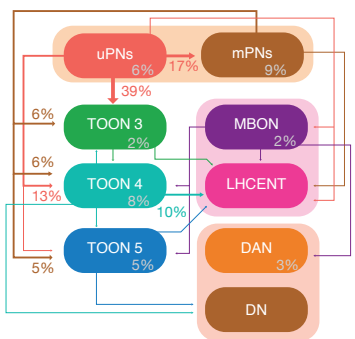

H glutamatergic connectivity

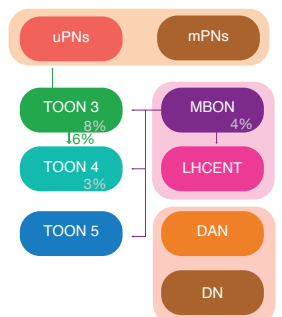

Figure 10-Figure supplement 1. Neuron class-level network diagrams of higher olfactory layers, broken down by neuron compartments and putative transmitters. A A circuit schematic of third-order olfactory neurons, showing the average connection strength between different classes of neurons (mean percentage of input synapses), broken into their layers, as well as the ALPN, LHCENT and MBON inputs to this system and DAN and DN outputs. The percentage in grey, within coloured lozenges, indicates the mean input that class provides to its own members. The threshold for a connection to be reported here is $5 \%$, and $>2 \%$ for a line to be shown. Subsequent plots just show a subset of this connectivity, i.e. B axo-dendritic connections, $\mathbf{C}$ axo-axonic connections, $\mathbf{D}$ dendro-dendritic connections, $\mathbf{E}$ dendro-axonic connections, $\mathbf{F}$ putative cholinergic connections, $\mathbf{G}$ putative GABAergic connections and $\mathbf{H}$ putative glutamatergic connections. 


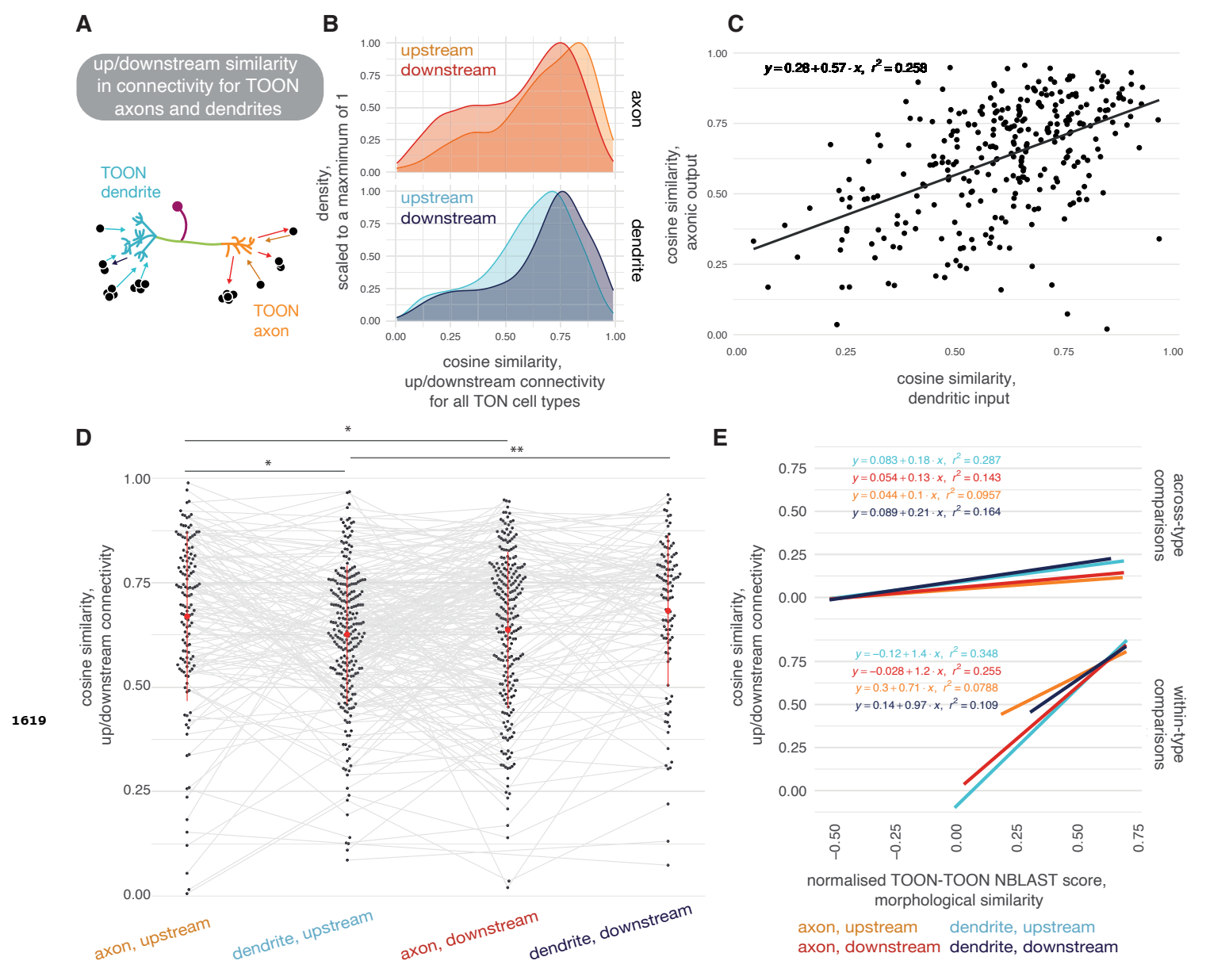

Figure 11-Figure supplement 1. Similarity in connectivity up and downstream of olfactory neurons. A Neuron can give and receive output from both their axons and their dendrites. B Density plots, showing cosine similarity scores for the cell types downstream of TOON-TOON pairs, where both members of the pair are from the same cell type. Upper, cosine similarity between the two populations upstream and downstream of the TOONs' axons. Lower, cosine similarity between the two populations upstream and downstream of the TOONs' dendrites. C Correlation between the mean cosine similarity between members of a TOON cell type's dendritic input populations $(x$-axis) and axonic target populations ( $y$-axis). D Cosine similarity between connections from/onto TOON axons/dendrites, for TOON-TOON pairs of the same cell type. $\mathbf{E}$ Correlations between morphological similarity and connectivity similarity shown, for both out-of-cell-type comparisons (top) and within-cell-type comparisons (bottom). Significance values: ns: $p>0.05 ; *$ : $p<=0.05 ; * *$ : $p<=$ $0.01 ; * * *: p<=0.001 ; * * * *: p<=0.0001$. 
bioRxiv preprint doi: https://doi.org/10.1101/2020.12.15.401257; this version posted May 14, 2021. The copyright holder for this preprint (which was not certified by peer review) is the author/funder, who has granted bioRxiv a license to display the preprint in perpetuity. It is made available under aCC-BY 4.0 International license.

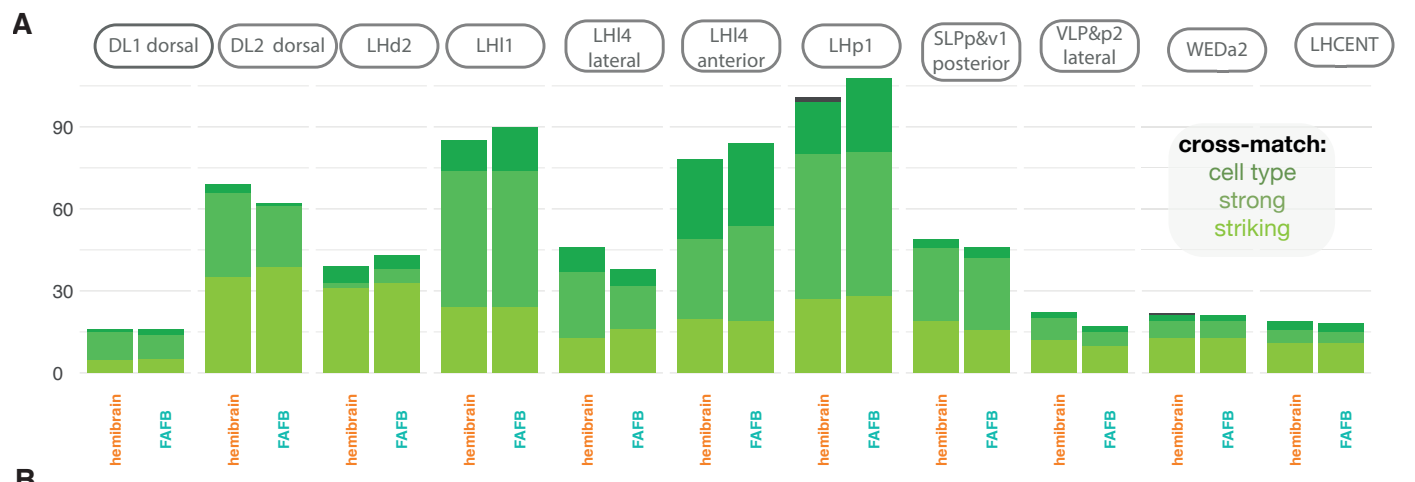

B
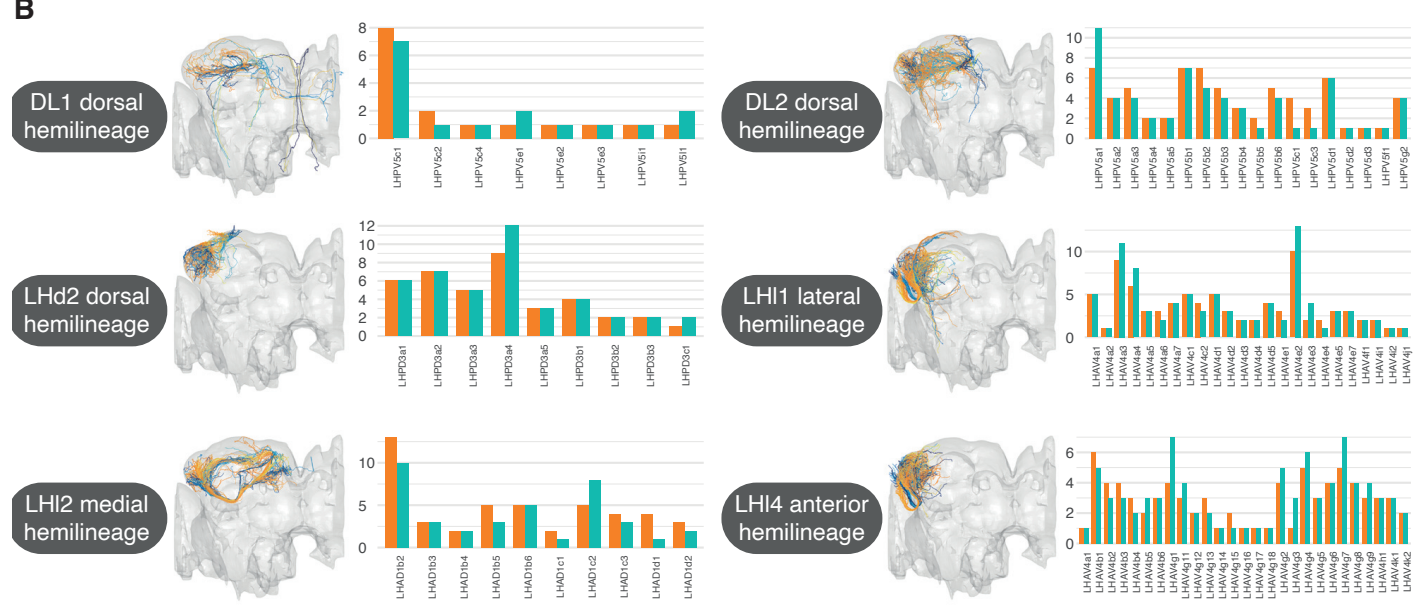

1620
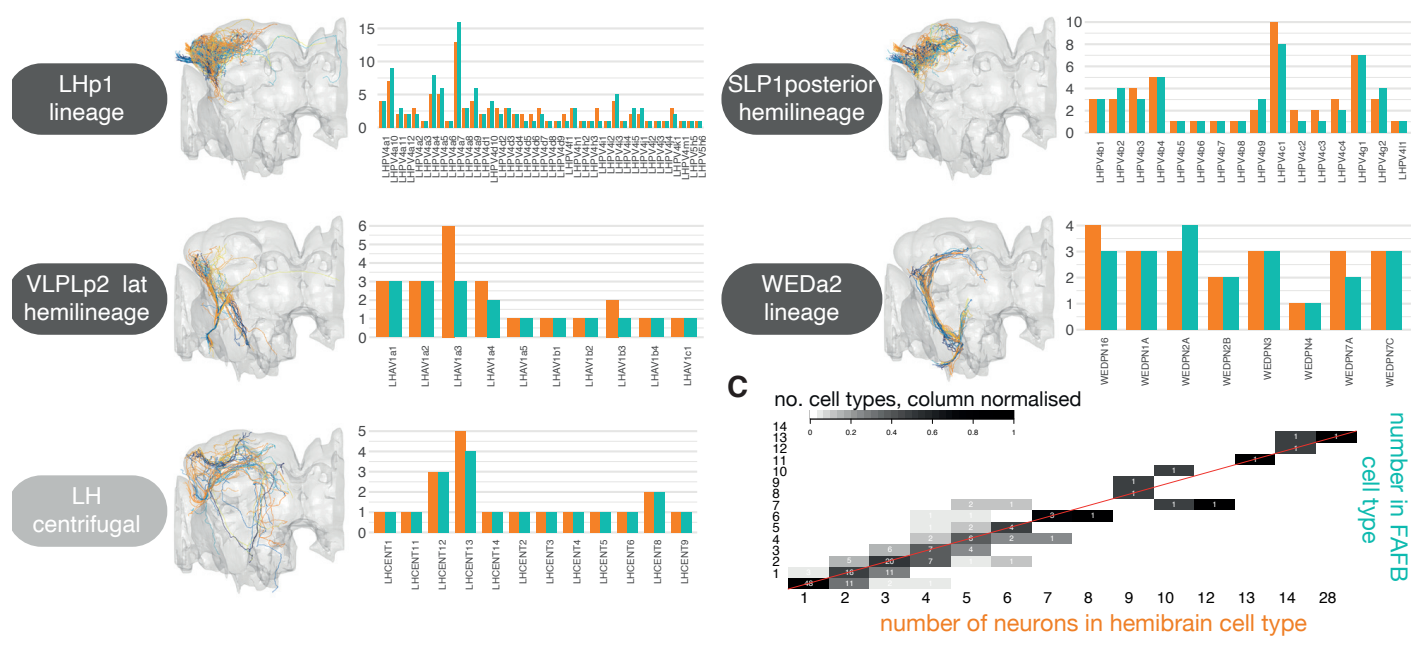

Figure 12-Figure supplement 1. A Tallies for the number of matches made from hemibrain $\rightarrow$ FAFB neurons (right) and hemibrain $\rightarrow$ FAFB neurons, and hemibrain $\rightarrow$ FAFB neurons (left), in both sets of 'secondary' hemilineages, plus LH centrifugal neurons, most of which are 'primary'. 'Striking' indicates that the two neurons look so similar they could be the 'same cell', 'strong' means that these cells look to belong to the same cell type, 'cell type' means that the two cells most likely belong to at least the same cell type. B Hemibrain image shows all reconstructed LHNs from both hemilineages are plotted together in the same brain space (hemibrain, grey) after a bridging registration had been applied (Bates et al., 2020a). Right, counts for neurons per identified LHN cell type, in each hemilineage in each data set. C Comparing the number of neurons in matched hemibrainFAFB cell types. Red unity line. 


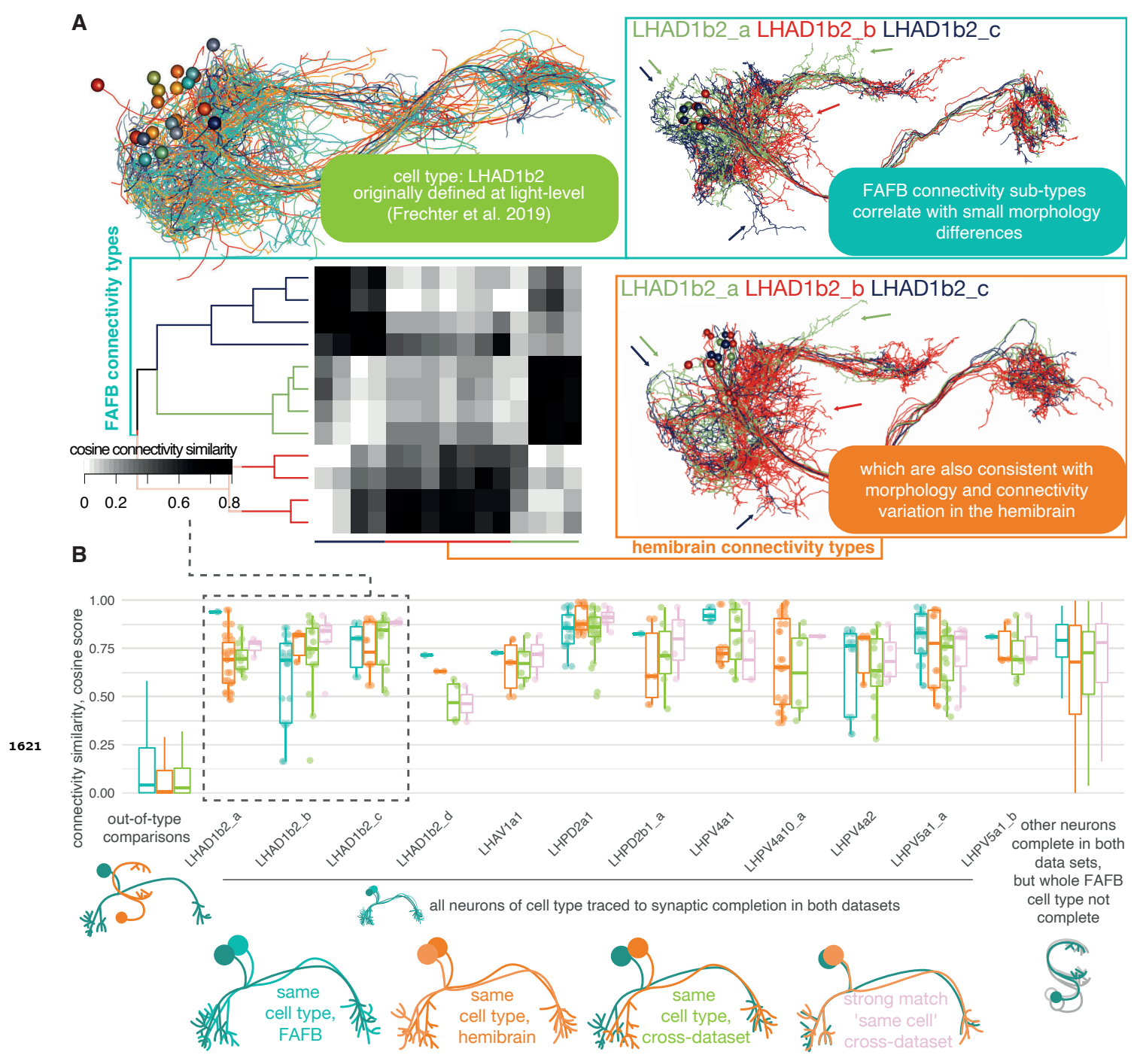

Figure 12-Figure supplement 2. Stereotypy in connectivity between lateral horn neurons in the hemibrain and FAFB. A An example of a cell type that looked cohesive at light-level resolution (Frechter et al., 2019), which actually breaks down into several connectivity sub-types on examination of the hemibrain data (Scheffer et al., 2020). Only uniglomerular ALPN (UPN) inputs are considered for the cross-correlation plot. B Cosine similarity scores for UPN -> LHN inputs. The cell types shown have been 'completely' synaptically reconstructed in both data sets (total of 34 FAFB reconstructions), and the cosine similarity score calculated for every pairing within each data set (FAFB, blue; hemibrain, orange), between the two data sets (green) and between all 'strongly' cross-data set matched pairs (pink). Each completed FAFB cell type comprises a mean of $3.4 \pm 1.1$ s.d. neurons. Out-of-cell type comparisons also made (leftmost), as well as for other neurons completed in FAFB, where not all members of the cell type have been completed (rightmost, 48 FAFB reconstructions) (Bates et al., 2020b). 

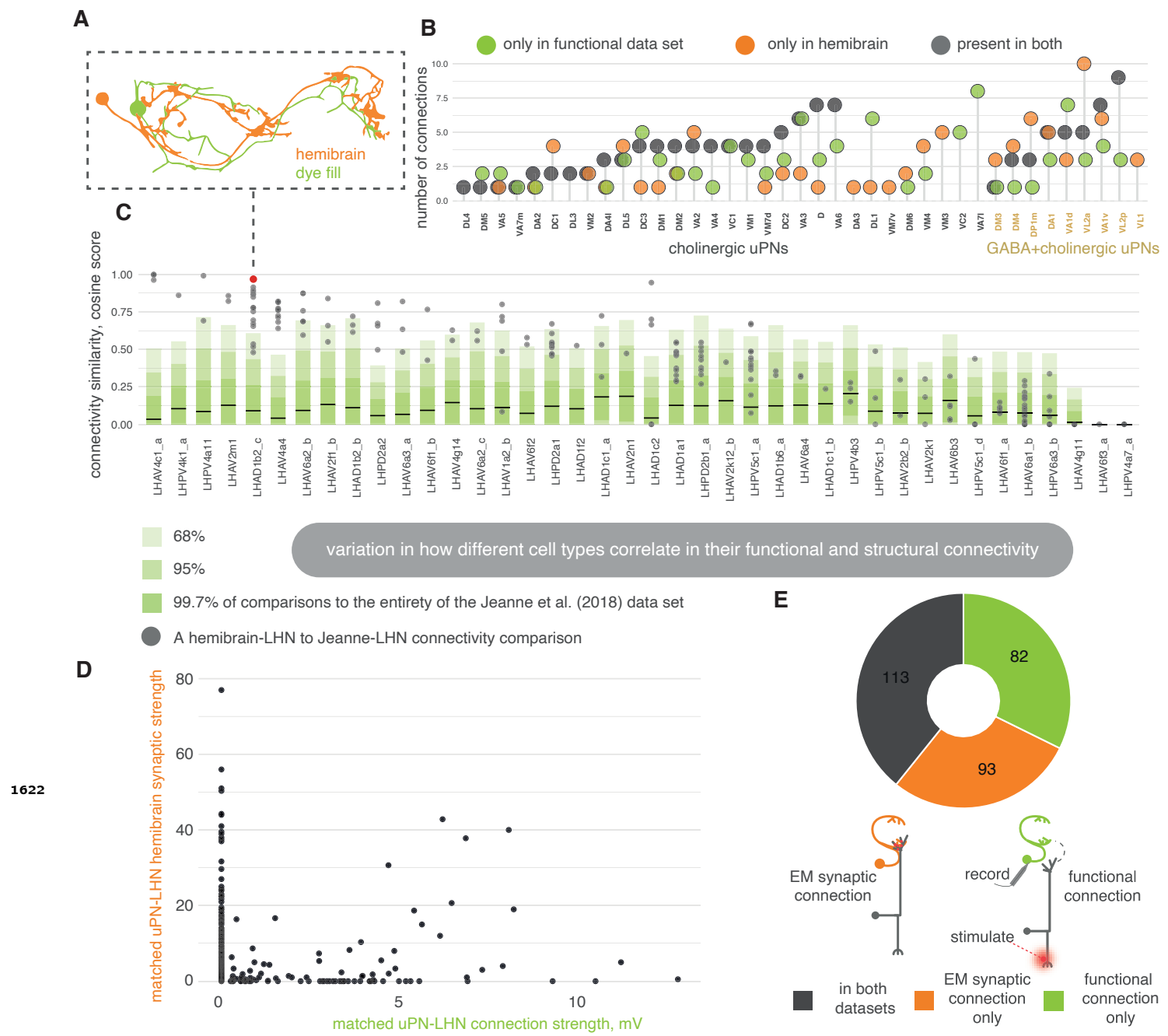

Figure 12-Figure supplement 3. Stereotypy in connectivity between lateral horn neurons in the hemibrain and a functional data set. A We matched light-level neuron skeletons from Jeanne et al. (2018) to hemibrain reconstructions; these light-level skeletons are associated with functional glomeruli $\rightarrow$ LHN connections ascertained by electrophysiology (Veanne et al., 2018). B We calculate the number of equivalent connections, present by any degree, between both data sets. C the cosine similarity score for ALPN $\rightarrow$ LHN connections. Horizontal bars, mean of the cosine comparison of each Jeanne et al. (2018) cell type against all other cells in the Jeanne et al. (2018) data set; dark green is one standard deviation from the mean, mid-green is two standard deviations, light green is three. Grey, comparison to matched hemibrain cell type, each point is one neuron-neuron comparison. D Scatter plot showing the strength of the recorded functional connections, in $\mathrm{mV}$, and the number of connecting synapses in their cross-matched hemibrain neurons, for the corresponding UPN $\rightarrow$ LHN contact. E The number of putative ALPN $\rightarrow$ LHN connections from a study on functional connectivity (Jeanne et al., 2018), that can be found in the hemibrain data set, for cross-matched neurons. A threshold of 4 synapses has been applied for the hemibrain data. 


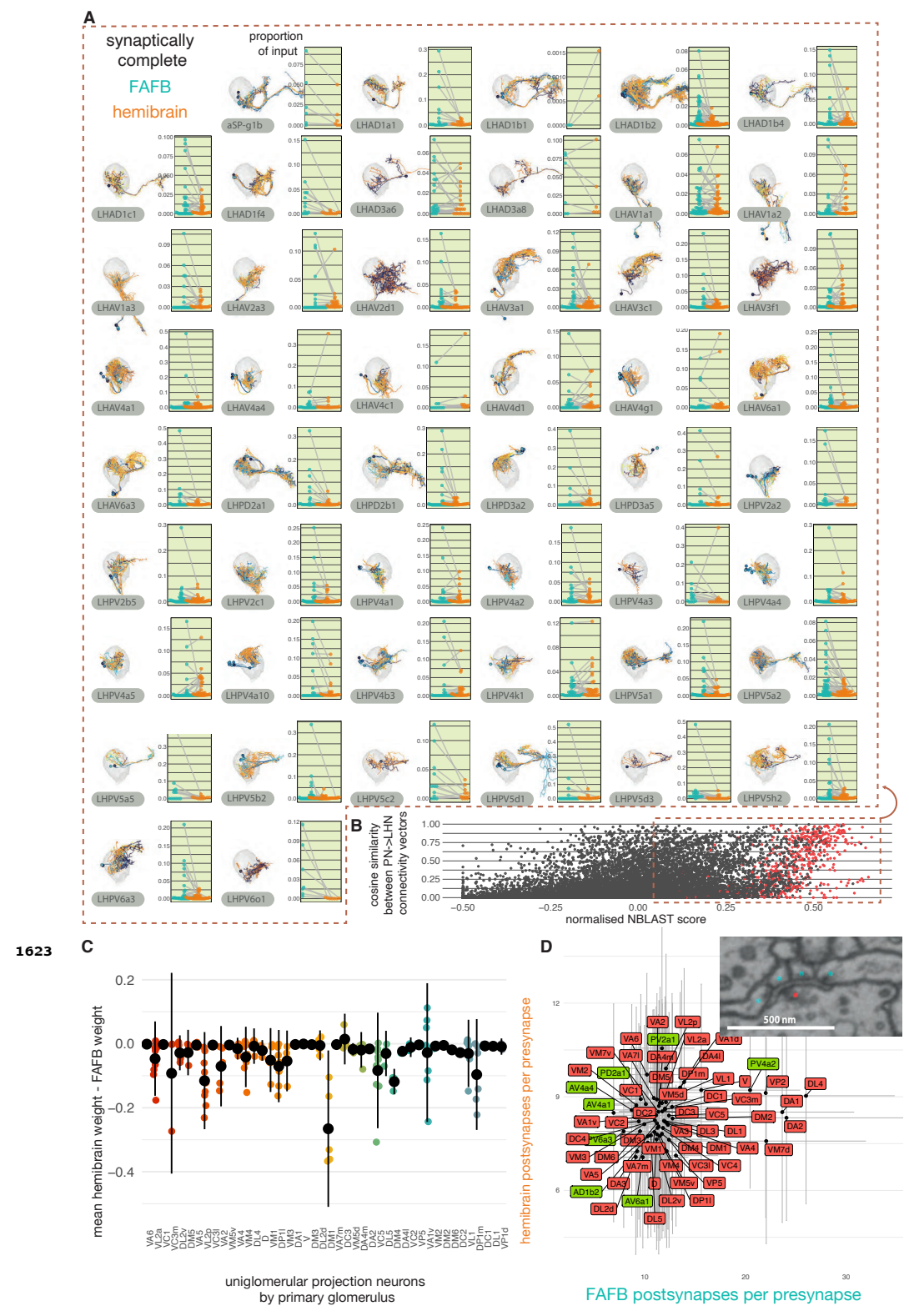

Figure 12-Figure supplement 4. Matching synaptically complete neurons between two EM data sets. A Each full hemibrain LHN cell type is compared with as many of its cognates in FAFB as possible, i.e. from those neurons reconstructed in Bates et al. (2020b). Each point represents the normalised connection strength of a single uPN type onto the target cell type in question (total connecting synapses / number of postsynapses in the target cell type). B Scatter plot showing the cosine similarity in UPN $\rightarrow$ LHN connectivity for LHN-LHN pairs, and LHN-LHN NBLAST scores. Every hemibrain neuron in A is compared with every FAFB neuron in A. Neurons of the same cell type are shown in red. C For each uPN cell type, the mean normalised connection strength to each hemibrain cell type is taken as in A, and the normalised connection strength to its cognate FAFB cell type is subtracted. Each point represents a different cell type comparison. $\mathbf{D}$ Inset, insect synapses are polyadic meaning that one presynaptic site connects with multiple postsynaptic sites. We previously manually marked up presynapse-postsynapse connections for dozens of presynapses over a limited number of cell types in FAFB (green) (Bates et alo, 2020b). The number of automatically detected postsynapses for each presynapse is also given for those same cell types in the hemibrain data set. 


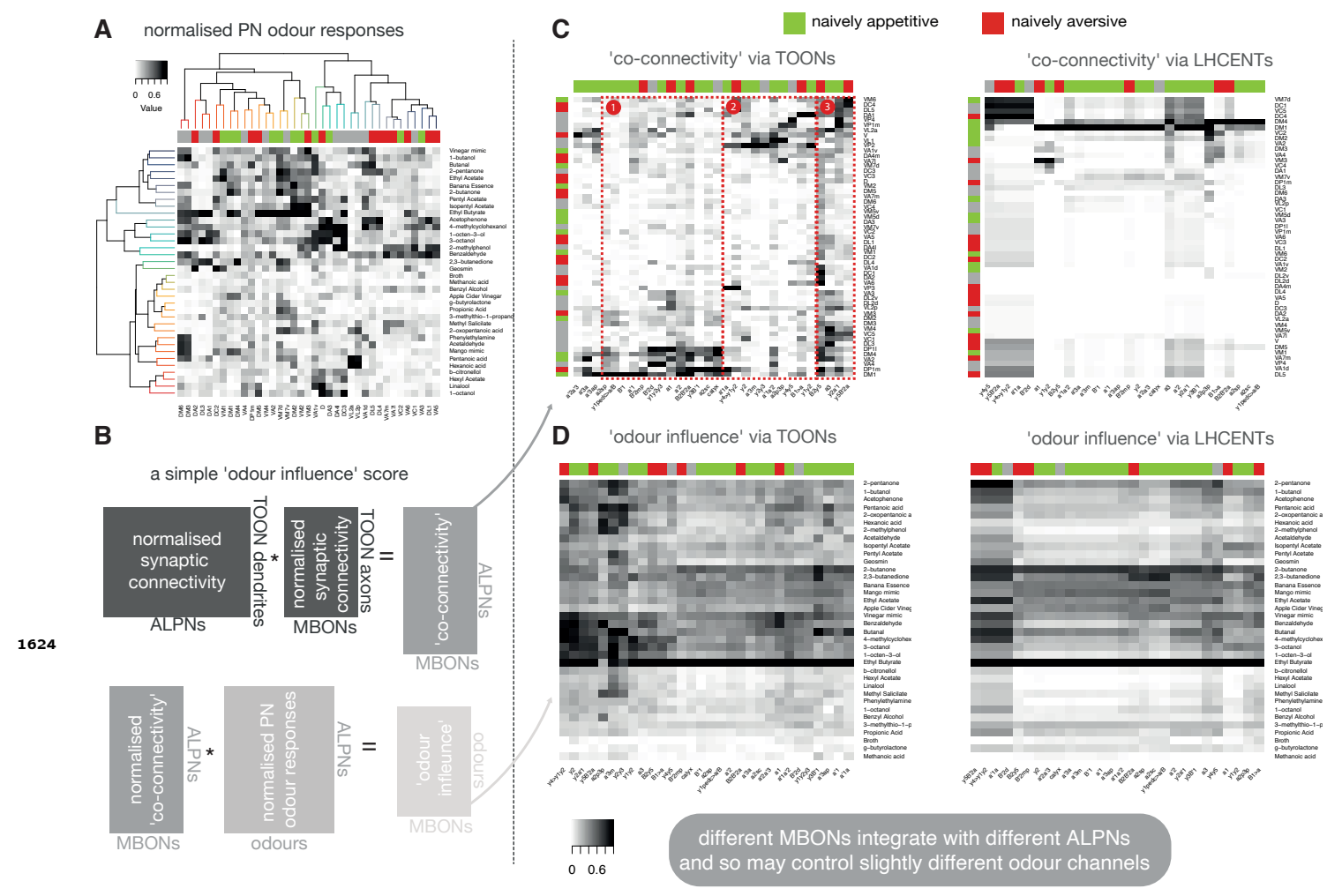

Figure 13-Figure supplement 1. Propagating known odour information to third-order olfactory neurons and mushroom body output neurons. A Calcium responses recorded from ALPN dendrites in the antennal lobe to odour presentations in Badel et al. (2016). B 'Co-connectivity' and 'odour influence' scores calculated by matrix multiplication of UPN $\rightarrow$ TOON or UPN $\rightarrow$ LHCENT connectivity, MBON connectivity and previously published odour response data (Badel et al., 2016). Groupings referred to in text labelled in red dashed boxes. 311 TOONs that have PN innervation at their dendrites and MBON innervation at their axons, were used. All matrices are minmax normalised across their columns. C,D Scores calculated using both MBON $\rightarrow$ TOON axon connectivity and MBON $\rightarrow$ LHCENT dendrite connectivity. 


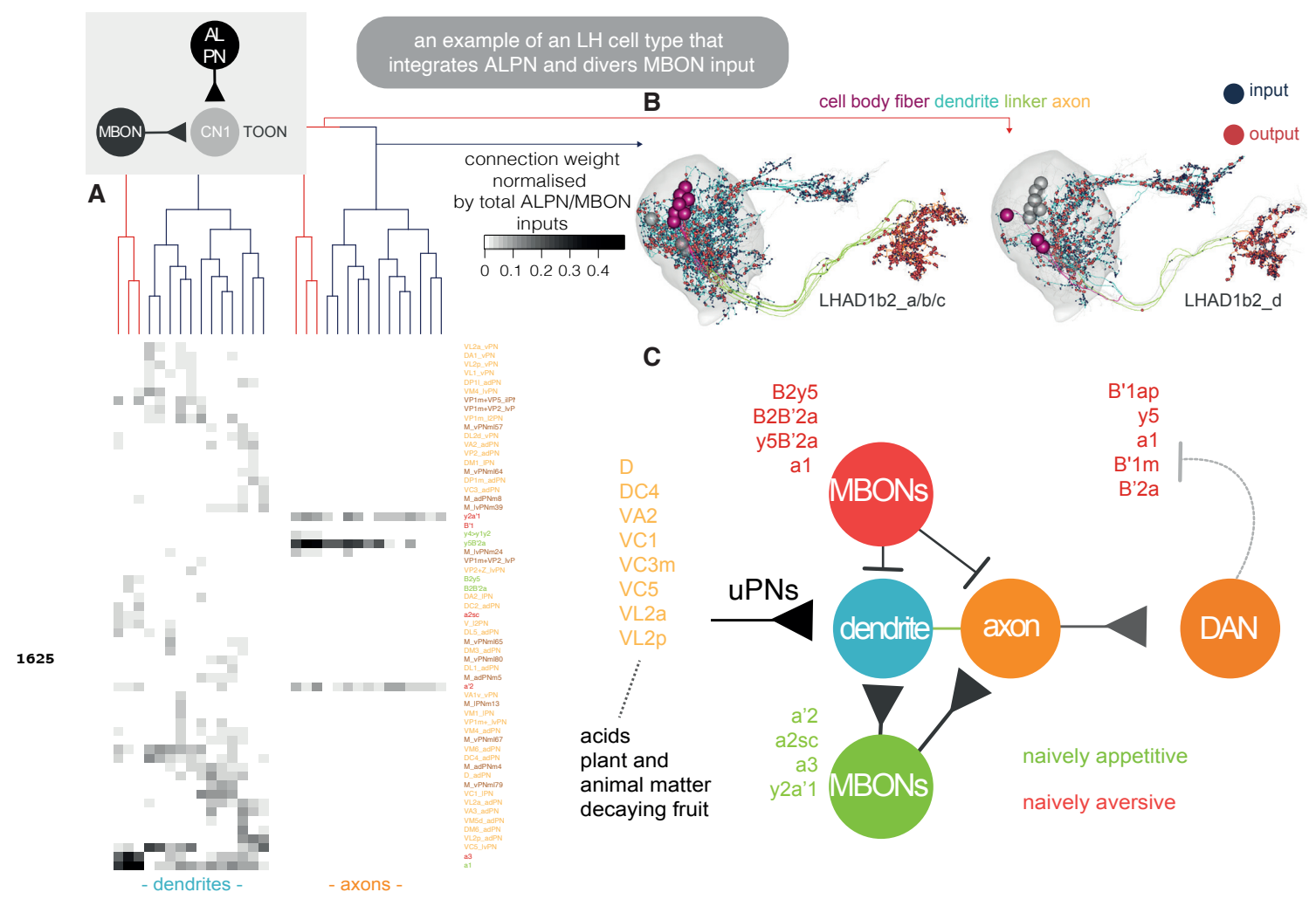

Figure 13-Figure supplement 2. An exemplar convergence cell type of the lateral horn and mushroom body. A Heatmap showing the normalised connectivity (weight / total number of LHN inputs) of ALPN and MBON input (rows) onto 15 LHAD1b2 neurons, axons (right) and dendrites (left). Clustering by Ward's method on dendrite data, cut at Euclidean linkage distance 0.2. MBONdendrite connects can happen on distinct sub-branches, see (Dolan et al., 2019). B Visualisation of the two connectivity clusters split into their dendrite-axon compartments (Schneider-Mizell et al., 2016; Bates et al., 2020b), which also correspond to small deviations in morphology. The other cluster is shown in grey in each panel. C An LHAD1b2 specific schematic for an emerging circuit motif integrating LH and MB output, based on the available labelled LHN data. MBONs coloured by naive valence, ALPNs by class. 


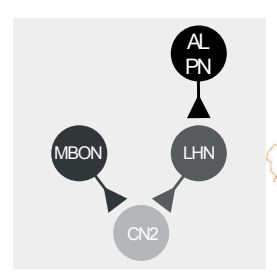

C

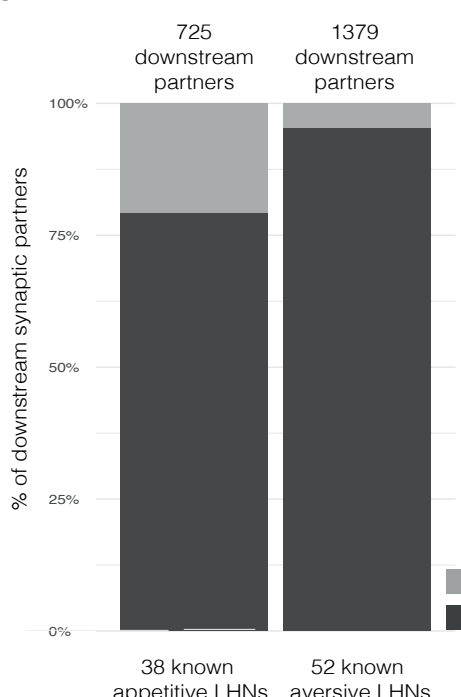

appetitive LHNs aversive LHNs

convergence neurons downstream of LHNs and MBONs

mostly receive excitation from known appetitive neurons

and inhibition from known aversive neurons
B recorded

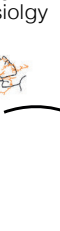

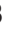

A - dye filld skeleton

$\mathrm{CN} 2$

not MBON target

D

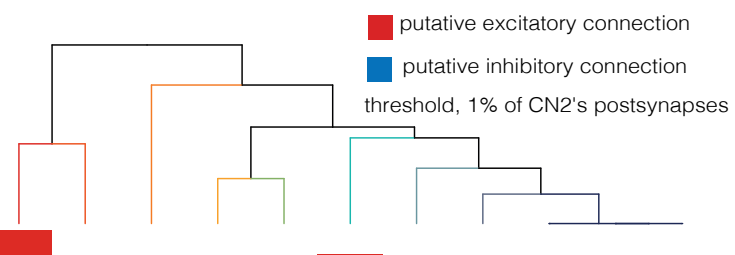

\begin{tabular}{l}
0 \\
Z \\
\hline \\
0 \\
0 \\
0 \\
0 \\
$\frac{0}{0}$ \\
0
\end{tabular}
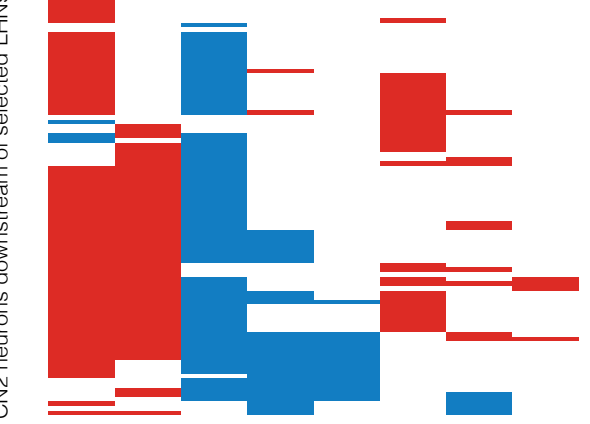
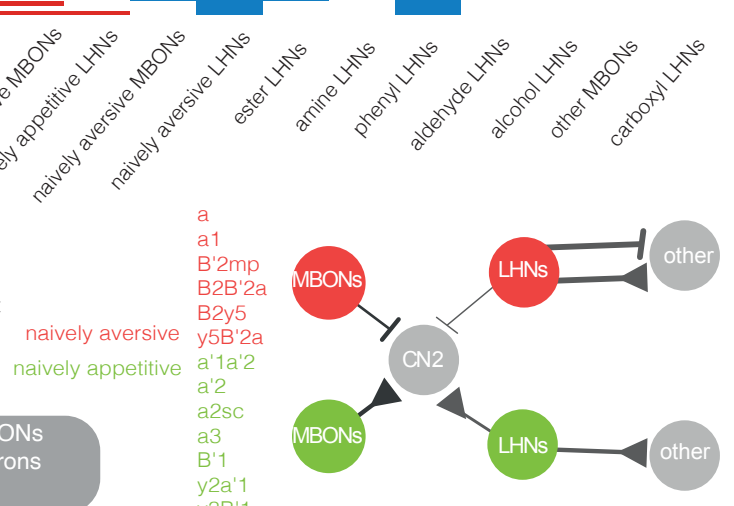

Figure 13-Figure supplement 3. Convergence neurons of the lateral horn and mushroom

body. A Matches were made between hemibrain reconstructions and LHN morphologies of electrophysiologically recorded cells (Frechter et al., 2019) and MultiColor FlpOut (Nern et al., 2015) data from LHN split-GAL4 lines used in behavioural studies (Dolan et al., 2019). A neuron is 'appetitive' if its optogenetic activation causes attraction to the stimulating light, and aversive if the opposite behaviour is significant (Dolan et al., 2019; Aso et al., 2014b). B Connections onto downstream targets (rows) by MBONs and LHNs, grouped by putative valence or odour coding. Note that LHN valence and odour coding categories are not mutually exclusive. Connections have been binarised: if the upstream neuron class accounts for greater than $1 \%$ of inputs onto a given target, the connection is shown. Putative excitatory connections in red (i.e. cholinergic) and inhibitory in blue (i.e. GABAergic or glutamatergic). C The proportion of downstream targets from putatively aversive and appetitive LHNs, that also receive direct MBON input. D A general schematic for an emerging circuit motif integrating LH and MB output, based on the available labelled LHN data. 
bioRxiv preprint doi: https://doi.org/10.1101/2020.12.15.401257; this version posted May 14,2021 . The copyright holder for this preprint (which

was not certified by peer review) is the author/funder, who has granted bioRxiv a license to display the preprint in perpetuity. It is made available under aCC-BY 4.0 International license.

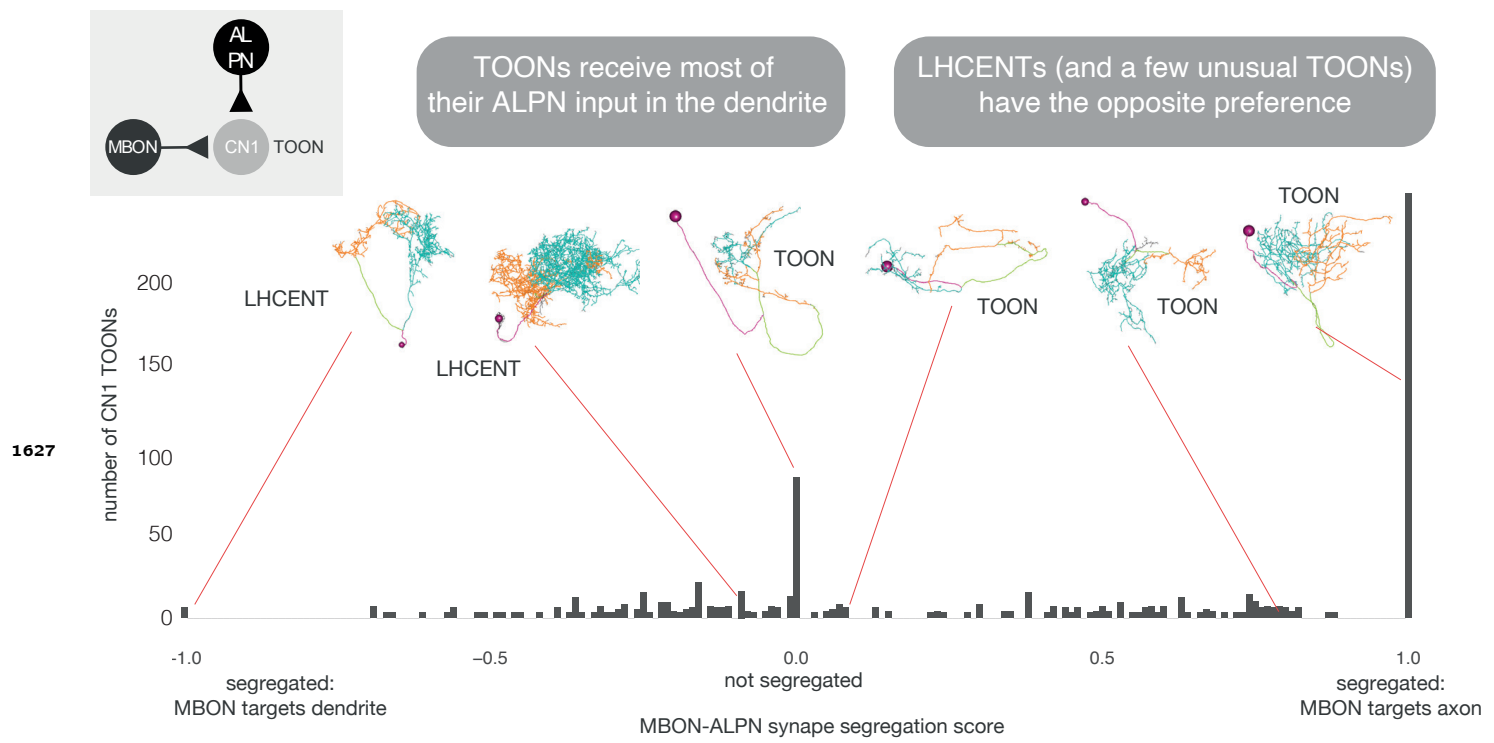

Figure 13-Figure supplement 4. A class-compartment separation score. The more positive the score, the more polarised the neuron such that ALPN innervation is seen at the dendrite and MBON innervation at the axon. Negative scores show the opposite segregation. See Methods. 


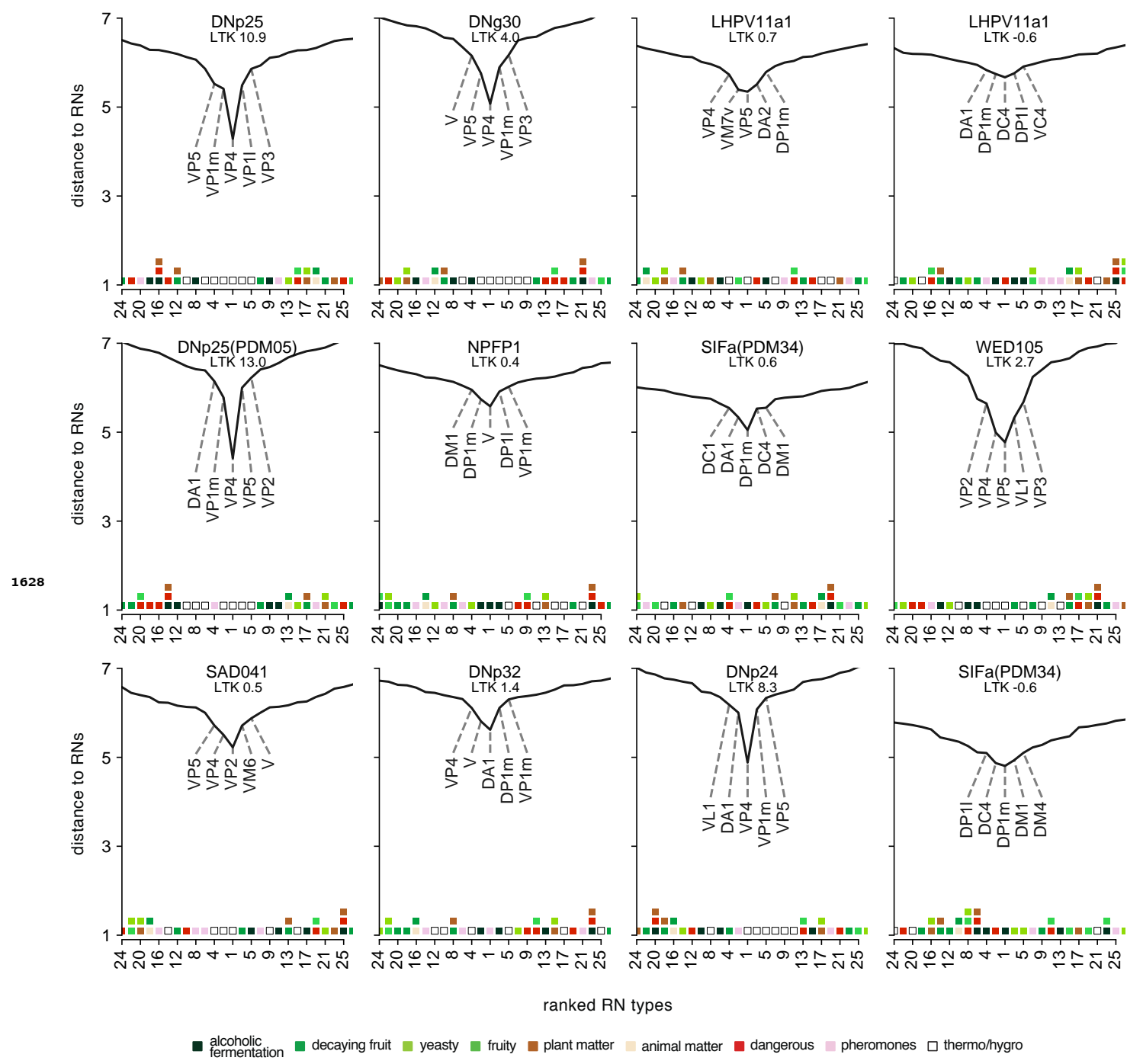

Figure 14-Figure supplement 1. Extended data for Figure 14E. ALRN $\rightarrow D N$ distances for $D N s$ not shown in main figure. A low distance indicates a more direct connection between an ALRN type and given DN. Only the top 25 ALRN types shown. Heatmap shows glomeruli odour scenes. 